
NBSIR 83-2725

,

\section{PERSPECTIVES IN HIGH ENERGY \\ NUCLEAR COLLISIONS}

Johann Rafelski

Institut fur Theoretische Physik der Universitat Frankfurt

D-6000 Frankfurt, West Germany

Michael Danos

U.S. DEPARTMENT OF COMMERCE

National Bureau of Standards

Center for Radiation Research

Washington, DC 20234

June 1983

U.S. DEPARTMENT OF COMMERCE, Malcolm Baldrigo, Secrotary NATIONAL BUREAU OF STANDARDS. Emeat Ambler, Director 

PERSPECTIVES IN HIGH ENERGY NUCLEAR COLLISIONS

\author{
Johann Rafelski \\ GSI, Darmstadt \\ and \\ Institut für Theoretische Physik der Universitat Frankfurt, \\ D-6000 Frankfurt ${ }^{\dagger}$ \\ and \\ Michael Danos \\ National Bureau of Standards, Washington, D.C. 20234
}

This report gives an overview of some aspects of hadronic physics relevant for the conception of a research facility devoted to the study of high energy nuclear collisions. Several concepts to be studied in nuclear collisions are selected, with emphasis placed on the properties and nature of the quark-gluon plasma, the formation of the plasma state in the central region and its anticipated lifetime, and the observability, through strangeness content of this new form of nuclear matter.

†Permanent address. Supported in part by Deutsche Forschugsgemeinschaft. 
Until now most of the investigations on the fundamental properties of matter have been performed in the lightest possible system. While this is an essential first step, there exist important phenomena based on many-body effects and which are not observable in the simple systems. At this time such a phenomenon is the hypothetical new phase of matter, viz., the quark-gluon plasma, which is that state where owing to a suitable combination of baryon density and temperature the individual hadrons have melted together, or, said differently, have dissolved, freeing the hadron constituents to form a weakly interacting Fermi and Bose gas. The existence of such a state of matter is an almost inevitable consequence of quantum chromodynamics (QCD) and the study of its properties is clearly of utmost importance [1].1 In this report we intend to present in reasonable detail our view of the present state of these developments, and also to provide some speculative extrapolations as guides to the planning of future experiments.

This new and exciting field of high energy physics is based on the hypothesis that the energy available in the collision of two relativistic heavy nuclei, at least in part of the system is equipartitioned among the accessible degrees of freedom. This means that there exists a domain in space in which, in a suitable Lorentz frame, the energy of the longitudinal motion has been largely transformed to transverse degrees of freedom. We call this region the 'fireball'. The physical variables characterizing a fireball are: energy density, baryon number density, and total volume. The basic question concerns the internal structure of the fireball. It can consist either of individual hadrons, or instead, of quarks and gluons in a new physical phase, the plasma, in which they are deconfined and can move freely over the volume of the fireball. It appears that the phase transition from the hadronic gas phase to the quark-gluon plasma is controlled mainly by the energy density of the fireball. Several estimates [2] lead to $0.5-1 \mathrm{GeV} / \mathrm{fm}^{3}$ for the critical energy density, to be compared with ca. $0.16 \mathrm{GeV} / \mathrm{fm}^{3}$ in nuclear matter. Many theoretical questions about strong interactions will be settled when the parameters and the nature of the phase transition are determined. We turn to these problems further below.

An important aspect of the developments in this field concerns the interaction of the experimentalists with the plasma. It seems that in order to observe the characteristics of the plasma one must either use electromagnetically interacting particles [3] (photons, lepton pairs) which can rather easily leave the plasma, or study the heavy flavor abundance generated in the collision [4]. To understand the latter point imagine that strange quarks are very abundant in the plasma (and indeed they are!). Then, since the (sss)state is bound and stable in the perturbative QCD-vacuum, it would be the most abundant baryon to emerge from the plasma. Surely the observation of such an "omegaization" of nuclear matter could not leave any doubts about the occurrence of the phase transition. The observation of other exotic hadrons [5] such as, e.g., csq, ${ }^{2} c \bar{s}$, etc. would support this conclusion. But even the enhancement of the more accessible abundance of $\bar{\Lambda}$ may already be sufficient at least for demonstrating the existence of a plasma.

${ }^{1}$ Numbers in brackets indicate literature references at the end of this paper.

${ }^{2}$ This so called $A^{+}$Baryon has recently been observed in $\Sigma^{-}-$Be interactions at $135 \mathrm{GeV} / \mathrm{C}$ [S. F. Biagi et al., Phys. Lett. 122B (1983) 455]. 
To continue to higher energy densities, one may speculate that the restoration of the perturbative QCD vacuum may be followed by the restoration of chiral symmetry, as shown qualitatively in figure 1.1, then of SU(2) symmetry, and finally of SU(5) symmetry. This way one could trace back the evolution of the universe [6] in the laboratory.

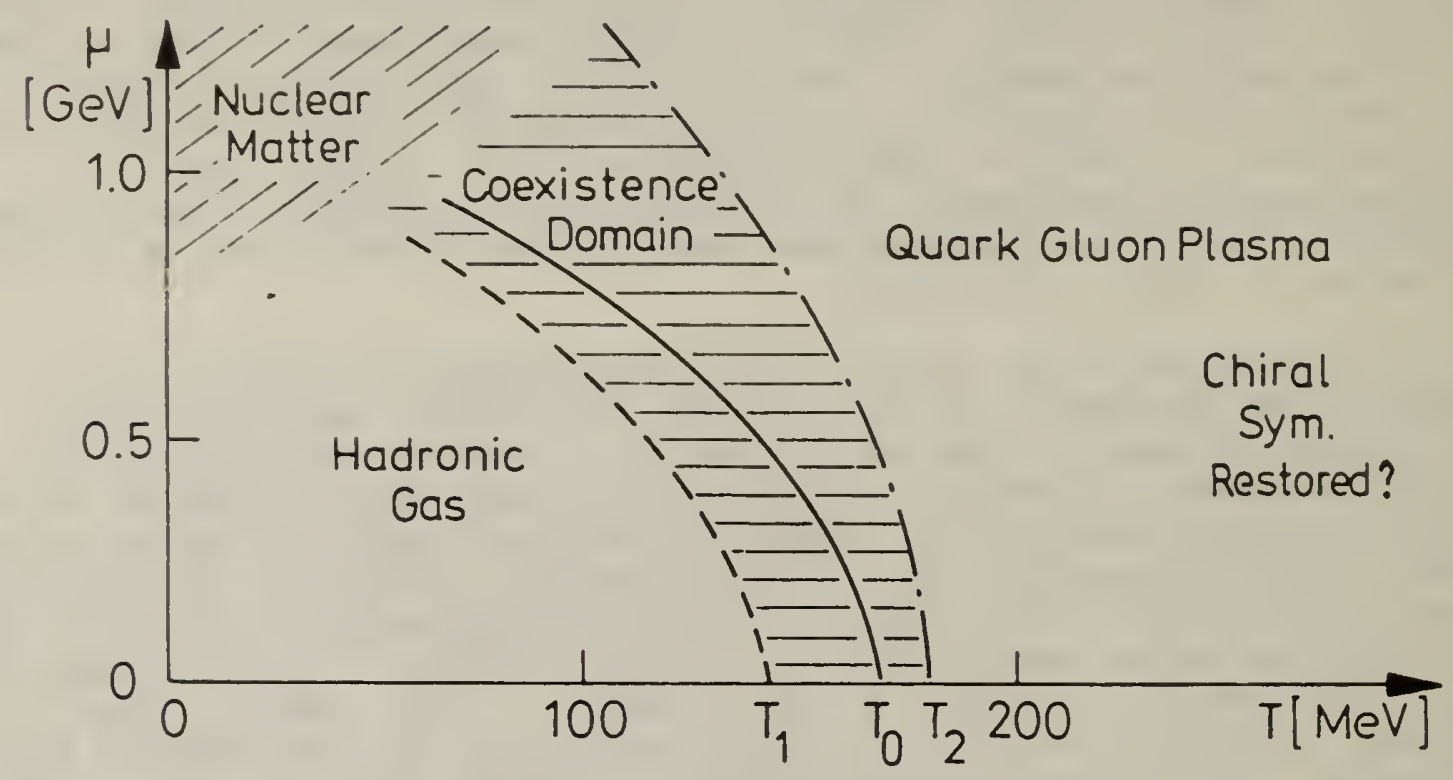

Fig. 1.1 Phase diagram of hadronic matter in the $\mu-T$ plane.

Another speculation concerns the fundamental aspect of the possible catalyzation of the baryon decay in the plasma [5]. A possible mechanism which has been discussed in the recent literature [7] involves the presence of magnetic monopoles. The quark-gluon plasma might just be the proper environment in which the catalyzer could continue to burn the baryon number at a rate sufficient to maintain the necessary particle densities and temperatures. However, in view of our ignorance of how precisely SU(5) tumbles down to SU(3) $\times$ SU(2) $\times U(1)$ we should be prepared for great surprises in this matter. Certainly, it would be most challenging to unlock the energy which had orginated in the Big Bang and has since remained frozen in the baryon number.

Coming back to earth we begin by recalling that in a statistical description of matter the un-handy microscopic variables, viz., energy, baryon number, etc. are replaced by thermodynamical quantities. To wit, the temperature $T$ is a measure of energy per degree of freedom; the baryon chemical potential $\mu$ controls the mean baryon density (see fig. 1.1). The statistical quantities such as entropy (= measure of the number of available states), pressure, heat capacity, etc. also will be functions of $T$ and $\mu$ and will have to be determined. The theoretical techniques required for the description of the two quite different phases, viz., the hadronic gas and the quark-gluon plasma, must allow for the formation of numerous hadronic resonances on the one side [8], which then at sufficiently high energy density dissolve into the state consisting of their constituents. At this point we must appreciate the importance and help in reaching the transition to the quark-gluon plasma 
provided by a finite, i.e., non-zero temperature. To obtain a high particle density, instead of compressing the matter (which as it turns out is quite difficult), we may heat it up; many plons are generated easily, allowing the transition to occur at moderate, even vanishing baryon density [9].

The paper is organized as follows. We begin by considering in section 2 the state of hadronic matter formed by individual baryons and mesons, which we call the hadronic gas phase. The present summary of the theoretical development of this field is based on the work of Hagedorn and Rafelski, to be published in greater detail elsewhere [9]. We content ourselves here with the presentation of the main results in so far as they influence our thinking about the phase transition to the quark-gluon plasma phase. We then turn in section 3 to a summary of the relevant postulates and results that characterize the current understanding of strong interactions in QCD. The most important postulate is that the true physical vacuum state in QCD is not the trivial perturbative state which is little changed when the interactions between quarks and gluons are turned off or on. In QCD the true vacuum state is believed to have a complicated structure which originates in the glue (gauge) sector of the theory. It is supposed not to permit the presence of color fields. The perturbative vacuum is an excited state with an energy density $B$ above the true vacuum. It is to be found inside nadrons where perturbative quanta of the theory, in particular quarks, can therefore exist. The occurrence of the true vacuum state is intimately connected with the glueglue interaction; gluons also carry the color charge that is responsible for the quark-quark interaction. The confinement of quarks is a natural consequence of the hypothetical structure of the true vacuum.

An important feature which arises as a consequence of the energy density $B$ of the perturbative vacuum is that the true vacuum exercises a pressure on the surface of the region of the perturbative vacuum. Indeed, this is just the idea of the original MIT bag model [10]. The Fermi pressure of the confined almost massless light quarks is in equilibrium with the vacuum pressure B. When many quarks are combined to form a giant quark bag then their properties inside the bag can be obtained using standard methods of many-body theory [2]. In particular, this also allows the inclusion of the effect of internal excitation through a finite temperature and through a change in the chemical composition. This will be discussed in section 4 .

The attentive reader might question our simultaneous use of the bootstrap model and the bag model to describe hadronic states. We will indeed find that the description of hadrons in terms of bound quark states on the one hand and the statistical bootstrap for hadrons on the other hand have many common properties and are quite complementary. Both, the statistical bootstrap and the bag model of quarks are based on quite equivalent phenomenological observations. While it would be most interesting to derive the phenomenological models quantitatively from the accepted fundamental basis - the Lagrangian quantum field theory of a nonabelian SU(3) "glue" gauge field coupled to colored quarks - we will have to content ourselves in this report with a qualitative understanding only. Already this will allow us to study the properties of hadronic matter in both aggregate states - with the emphasis put in this report in particular on the state in which individual hadrons have dissolved into the plasma consisting of quarks and of the gauge field quanta, the gluons. 
Having described the properties of both hadronic phases, we present in section 5 a discussion of the possible production and lifetime of the baryon rich plasma in nuclear collisions in the central kinematic region and describe the phase transition between the hadron gas and the plasma in section 6 . As opposed to the prevailing opinion [11] according to which the baryons populate the fragmentation region while the plasma has very low baryon density and is formed at central rapidity, we describe a likely, even if perhaps rare, reaction mechanism in which both energy and baryon number remain together in the central rapidity region [12]. The conventional description is based on extrapolations of $\mathrm{pp}$ and $\mathrm{pA}$ collisions, which in our view cannot lead to the pileup of matter, i.e., baryon number, which is needed in our description. In order to estimate the evolution of the plasma state we must consider, also contrary to popular belief [13] that hydrodynamical expansion dominates the plasma evolution, we show that the losses arising from particle radiation through the plasma surface [14] are important and, indeed, determine the time evolution of the baryon-rich plasma.

In section 7 we discuss the role of strangeness as a characteristic observable of the plasma state [4] with particular emphasis on strangeness generation in the plasma by elementary processes, and on expectations about the normal hadronic gas phase. From the comparison of the expectations for both phases of hadronic matter we are lead to propose the study of strangeness abundance as a possible approach to the observation of the properties and parameters of the quark-gluon plasma created in nuclear collisions. This situation is emphasized in section 8 , in which it is suggested that generally speaking, for several quantitatively known effects strangeness is an excellent experimental trigger for the presence of plasma droplets in light-ion-nucleus, or slow p-nucleus collisions.

\section{THERMODYNAMICS OF INTERACTING HADRONS}

The main hypothesis which allows us to simplify the description is to postulate the dominance of the hadron-hadron interaction by the hadron resonances [8]. In this case the hadronic gas phase is essentially a superposition of an infinity of different hadronic gases and all information about the interaction is hidden in the mass spectrum $\tau\left(m^{2}, b\right)$ which describes the number of hadrons of baryon number $b$ in a mass interval $\mathrm{dm}^{2}[9]$.

\section{Let us first assume that the mass spectrum $\tau\left(m^{2}, b\right)$ is already known.} Then, following the developments of Refs. [8],[9], the grand canonical level density $\sigma$ is given by an invariant phase space integral. The extreme richness of the spectrum, $\tau\left(\mathrm{m}^{2}, \mathrm{~b}\right) \sim \exp \left(\mathrm{m} / \mathrm{T}_{0}\right)$, enables us to neglect Fermi and Bose statistics above $T \approx 50 \mathrm{MeV}$ and to treat all particles as "Boltzmannions" in the external volume $V_{\text {ex }}$. We find for given $p_{\mu}=(E, \vec{p})$ and baryon number $b$ $\left(\delta_{K}=\right.$ Kronecker $\delta$-function)

$$
\begin{aligned}
\sigma\left(p, V_{e x}, b\right) & =\delta^{4}(p) \delta_{K}(b) \\
& +\sum_{N=1}^{\infty} \frac{1}{N !} \delta^{4}\left(p-\sum_{i=1}^{N} p_{i}\right) \sum_{\left\{b_{1}\right\}} \delta_{K}\left(b-\sum_{i=1}^{N} b_{i}\right) \sum_{i=1}^{N} \frac{2 \Delta_{\mu} p_{i}^{\mu}}{(2 \pi)^{3}} \tau\left(p_{i}^{2}, b_{i}\right) d^{4} p_{i} \cdot(2.1)
\end{aligned}
$$


Here, the first term corresponds to the vacuum state. The $N^{\text {th }}$ term is the sum over all possible partitions of the total baryon number and the total momentum p among $N$ Boltzmannions, each having an internal number of quantum states given by $\tau\left(p_{j}^{2}, b_{j}\right)$. These Boltzmannions are hadronic resonances of baryon number $b_{i}\left(-\infty<b_{j}<\infty\right)$. Every resonance can move freely in the remaining volume $\Delta$ left over after subtracting the proper volumes $V_{C}$ of all hadrons from the external volume $V_{\text {ex }}$ :

$$
\Delta^{\mu}=v_{e x}-\sum_{i=1}^{N} v_{c, i}^{\mu}
$$

$V^{\mu}$ is a covariant generalization of $V_{j}$; in the rest frame $V_{\mu}=(V, 0)$.

In the generalization (2.1) of the familiar phase space formula, three essential features of the hadronic interactions are now explicitly included:

a) The dominance of the particle scattering by the dense set of hadronic resonances via $\tau\left(m_{i}^{2}, b_{i}\right)$.

b) The proper natural volumes of hadronic resonances via $\Delta^{\mu}$.

c) The conservation of baryon number and the clustering of hadrons into lumps of matter with $|b|>1$.

The thermodynamic properties of a hot hadronic gas follow from the study of the grand partition function $Z(\beta, V, \lambda)$, as obtained from the level density $\sigma(p, v, b)$ :

$$
Z(\beta, V, \lambda)=\sum_{b=-\infty}^{\infty} \lambda^{b} \int e^{-\beta \cdot p} \sigma(p, V, b) d^{4} p
$$

Here the covariant generalization of thermodynamics with the inverse temperature four-vector $\beta_{\mu}$ has been used. In the rest frame of the relativistic baryon the chemicat potential $\mu$ is defined by

$$
\lambda=\exp (\mu / T) ;
$$

it is introduced in order to conserve the baryon number in the statistical ensemble. All quantities of physical interest can be derived as usual by differentiating $\mathrm{enZ}$ with respect to its variables.

Equations (2.1)-(2.3) leave us with the task of finding the mass spectrum $\tau$. Experimental knowledge of $\tau$ is limited to low excitations and/or low baryon number. Hagedorn [8] has introduced a theoretical model: "the statistical bootstrap," in order to obtain a mass spectrum consistent with direct and indirect experimental evidence. The qualitative arguments leading to an integral equation for $\tau\left(m^{2}, b\right)$ are the following. When $V_{\text {ex }}$ in eq (2.1) is just the proper volume $V_{C}$ of a hadronic cluster then, up to a normalization factor 
$\sigma$ in eq (2.1) is essentially the mass spectrum $\tau$. Indeed, how could we distinguish between a composite system as described by eq (2.1) compressed to the natural volume of a hadronic cluster and an "elementary" cluster having the same quantum numbers? Thus we demand

$$
\left.\sigma(p, V, b)\right|_{V=V_{C}} \equiv H \tau\left(p^{2}, b\right)
$$

where the "bootstrap constant" $H$ is to be determined below. It is not sufficient simply to insert eq (2.5) into eq (2.1) to obtain the bootstrap equation for $\tau$; more involved arguments are necessary [9b] in order to obtain the following "bootstrap equation" for the mass spectrum:

$$
\begin{aligned}
H \tau\left(p^{2}, b\right) & =H z_{b} \delta_{0}\left(p^{2}-M_{b}^{2}\right) \\
& +\sum_{N=2}^{\infty} \frac{1}{N !} \int \delta^{4}\left(p-\sum_{i=1}^{N} p_{i}\right) \sum_{\left\{b_{1}\right\}} \delta_{K}\left(b-\sum_{i=1}^{N} b_{i}\right) \sum_{i=1}^{N} H \tau\left(p_{i}^{2}, b_{i}\right) d^{4} p_{i} .
\end{aligned}
$$

The first term is the lowest one-particle contribution to the mass spectrum, $\mathrm{z}_{\mathrm{b}}$ is its statistical weight $(2 \mathrm{I}+1)(2 \mathrm{~J}+1)$. The index " 0 " restricts the $\delta$ function to the positive root. Only terms with $b=0, \pm 1$, corresponding to lowest energy qq (pion) and qqq (nucleon) states contribute in the first term of eq (2.6). All excitations are contained in the second term since arbitrary quark configurations can be achieved by combining $\left[(q \bar{q})^{n}\right.$ (qqq) $\left.^{m}\right]$. The small influence of heavy flavors is ignored at this point but easily can be introduced.

In the course of deriving the bootstrap equation (2.6) it turns out that the cluster volume $V_{c}$ grows proportional to the invariant cluster mass [9],

$$
V_{C}\left(p^{2}\right)=\sqrt{p^{2}} /(4 B)
$$

The proportionality constant has been $c a l l e d 4 B$ in order to establish a close relationship with the quark bag model [10]. The value of $B$ can be derived from different considerations involving the true and perturbative QCD states. While the original MIT-bag fit has been $\mathrm{B}^{1 / 4}=145 \mathrm{MeV}$, the (unweighted) average of different fits is today

$$
\begin{aligned}
B^{1 / 4} & =190 \mathrm{MeV} \\
B & =170 \mathrm{MeV} / \mathrm{fm}^{3}
\end{aligned}
$$

As $f a r$ as the bootstrap is concerned the constant $H$ and the bag constant $B$ are free parameters. However, as just pointed out, $B$ is determined from other considerations, while $H$ turns out to be inversly proportional to $B$ [9b]. 
Hence, if one wishes to believe the statistical bootstrap approach to the last detail there remains no free parameter in this approach. The implications of this for the transition gas to plasma will now be discussed. $[8]$,

Instead of solving eq (2.6), which leads to the exponential mass spectrum

$$
\tau\left(m^{2}, b\right) \sim e^{m / T_{0}}
$$

we wish to concentrate here on the double integral, i.e., the Laplace transform of eq (2.6) which will be all we need to estabiish the physical properties of the hadronic gas phase. Introducing the transforms of the oneparticle term, eq (2.6)

$$
\phi(\beta, \lambda)=\sum_{b=-\infty}^{\infty} \lambda^{b} H z_{b} \delta_{0}\left(p^{2}-M_{b}{ }^{2}\right) e^{-\beta \cdot d} d^{4} p
$$

with pions and nucleons only ( $K_{n}$ is the modified Bessel function)

$$
\varphi(\beta, \lambda)=2 \pi H T\left[3 m_{\pi} K_{1}\left(\frac{m}{T}\right)+4\left(\lambda+\frac{1}{\lambda}\right) m_{N} K_{1}\left(\frac{m_{N}}{T}\right)\right],
$$

and the mass spectrum:

$$
\phi(\beta, \lambda)=\sum_{b=-\infty}^{\infty} \lambda^{b} \int H \tau\left(p^{2}, b\right) e^{-\beta \cdot d} d^{4} p,
$$

we find for the entire eq (2.6) the simple relation

$$
\phi(\beta, \lambda)=\varphi(\beta, \lambda)+\exp [\phi(\beta, \lambda)]-\phi(b, \lambda)-1 .
$$

To study the behavior of $\phi(\beta, \lambda)$ we make use of the apparent implicit dependence:

$$
\phi(\beta, \lambda)=G(\varphi(\beta, \lambda))
$$

with the function $G$ being defined by eq (2.13)

$$
\varphi=2 G+1-\exp [G]
$$


This function $G(\varphi)$ is shown in figure 2.1. As is apparent there is a maximal $v$ alue $\varphi_{0}$

$$
\varphi_{0}=\ln (4 / e)=0.3863
$$

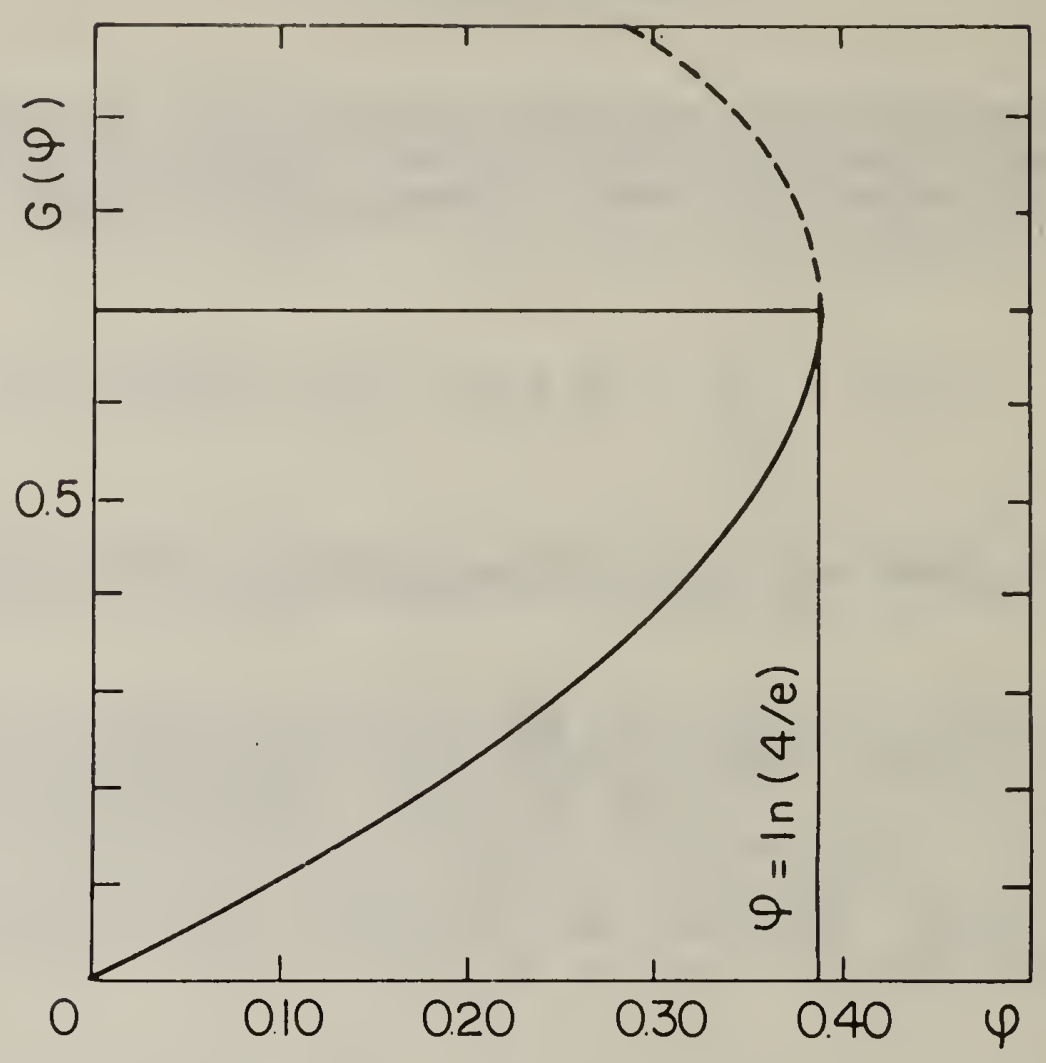

Fig. 2.1 Bootstrap function $G(\varphi)$. The dashed line represents the unphysical
branch. beyond which the function $G$ has no real solutions. Recalling the physical meaning of $G$, eq $(2.12,2.14 a)$ we conclude that eq $(2.14 c)$ establishes a boundary for the values of $\lambda$, $i . e ., \mu, \mu$, and $T$ beyond which the hadronic gas phase cannot exist. This boundary is implicitly given by the relation (2.11):

$$
\ln (4 / e)=2 \pi H T_{c r}\left[3 m_{\pi} K_{1}\left(\frac{m}{T_{c r}}\right)+8 \cosh \left(\frac{\mu_{c r}}{T_{c r}}\right) m_{N} K_{1}\left(\frac{m^{m} N}{T_{c r}}\right)\right]
$$

shown in figure 2.2. The region denoted "Hadronic Gas Phase" is described by our current approach. With $H$ correlated to $B$ as given by eq $(2.8)$ we find

$$
T_{c r}\left(\mu_{c r}=0\right)=T_{0} \sim 160-170 \mathrm{MeV} .
$$




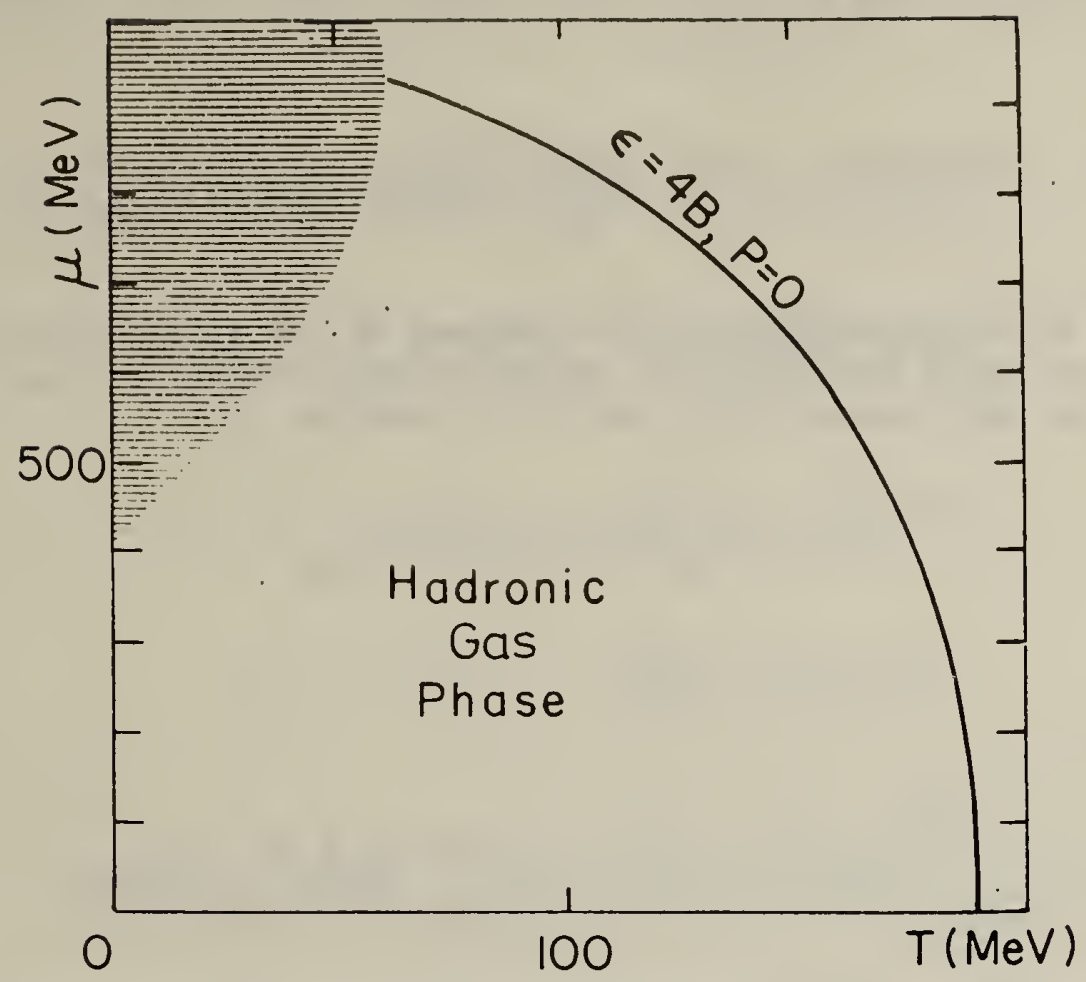

Fig. 2.2 Boundary of the "hadronic gas phase" in the bootstrap model. In the shaded region quantum statistics cannot be neglected.

However, in view of the uncertainties involved it is more prudent to argue that the value $\mathrm{T}_{\mathrm{cr}} \sim 160-170 \mathrm{MeV}$ which is required in the description of hadronic reactions determines the value of the parameter $H$. Note that $\mu=0$ implies zero baryon number of the plasma state. For $\mu_{0}=\mu_{c r}\left(T_{c r}=0\right)$ the solution of eq (2.15) is simply $\mu_{c r} \sim m_{N}$ since no quantum statisfics effects have been included. Thus the dashed region in figure 2.2 "nuclear matter" is excluded from our considerations. As we shall see shortly, the boundary to the hadronic gas phase is also characterized by a constant energy density $\varepsilon=4 B$.

Given the function $G(\varphi)=\phi(\beta, \lambda)$ we can in principle study the form of the hadronic mass spectrum. As it turns out we can obtain the partition function directly from $\phi$. Namely, the formal similarity between eq (2.3) and eq (2.12) can be exploited to derive a relation between their integral

transforms [9] (from here on: $\beta=\sqrt{\beta_{\mu} \beta^{\mu}}$ )

$$
\ln Z\left(\beta, V_{\text {ex }}, \lambda\right)=-\frac{2 \Delta\left(V_{\text {ex }}\right)}{H(2 \pi)^{3}} \frac{\partial}{\partial \beta} \phi(\beta, \lambda)
$$


which can also be written in a form which makes more explicit the different physical inputs:

$$
\ln Z\left(\beta, V_{\text {ex }}, \lambda\right)=\frac{\Delta\left(V_{\text {ex }}\right)}{V_{\text {ex }}} \frac{\partial G(\varphi)}{\partial \varphi} Z_{1}(\beta, \lambda, V)
$$

In the absence of a finite hadronic volume and of the interactions described by the first two terms respectively, we would simply have an ideal Boltzman gas described by the one-particle partition function $Z_{1}$ :

$$
z_{1}=z_{q \vec{q}}+2 \cosh (\mu / T) z_{q q q}
$$

where

$$
Z_{q \bar{q} / q q q} \times(2 I+1)(2 S+1) \frac{V T^{3}}{2 \pi^{2}}\left(\frac{m}{T}\right)^{2} k_{2}\left(\frac{m / N}{T}\right) .
$$

The remainder of the discussion of the hadronic gas is an application of the rules of statistical thermodynamics. However, when working out the relevant physical consequences we must always remember that the fireball is an isolated physical system for which the statistical approach has been taken in view of the internal disorder (high number of available states) rather than because of a coupling to a heat bath. Let us first discuss the role of the available volume. As we have explicitly assumed, all hadrons have an internal energy density $4 B$ (actually at finite pressure there is a small correction, see Ref. [4a] for details). Hence the total energy of the fireball $E_{F} c a n$ be written as

$$
E_{F} \equiv \varepsilon V_{e x}=4 B\left(V_{e x}-\Delta\right)
$$

where $V_{\text {ex }}$ - $\Delta$ is the volume occupied by the hadrons. We thus find

$$
\Delta=V_{\text {ex }}-E_{F} / 4 B=V_{e x}(1-\varepsilon /(4 B)) .
$$

By investigating the meaning of the thermodynamic averages it turns out that the apparent $(\beta, \lambda)$ dependence of the available volume $\Delta$ in eq $(2.22)$ must be disregarded when differentiating $2 n Z$ with respect to $B$ and $\lambda$. As eq (?..1) shows explicitly, the density of states of extended particles in $V_{\text {ex }}$ is the same as that of point particles in $\Delta$. Therefore also 


$$
\operatorname{lnZ}\left(\beta, V_{e x}, \lambda\right) \equiv \ln Z_{p t}(\beta, \Delta, \lambda) .
$$

We thus first calculate the point particle energy, and baryon number densities, pressure, and entropy density

$$
\begin{aligned}
& \varepsilon_{p t}=-\frac{1}{\Delta} \frac{\partial}{\partial \beta} \ln Z_{p t}=\frac{2}{H(2 \pi)^{3}} \frac{\partial^{2}}{\partial \beta^{2}} \phi(\beta, \lambda) \\
& \nu_{p t}=\frac{1}{\Delta} \lambda \frac{\partial}{\partial \lambda} \ln Z_{p t}=-\frac{2}{H(2 \pi)^{-3}} \lambda \frac{\partial^{2}}{\partial \lambda \partial \beta} \phi(\beta, \lambda) \\
& P_{p t}=\frac{T}{\Delta} \ln Z_{p t}=-\frac{2 T}{H(2 \pi)^{3}} \frac{\partial}{\partial B} \phi(B, \lambda) \\
& s_{p t}=\frac{1}{\Delta} \frac{\partial}{\partial T}\left(T \ell n Z_{p t}\right)=\frac{P_{p t}}{T}+\frac{\varepsilon_{p t}-\mu \nu p t}{T} .
\end{aligned}
$$

From this, we easily find the energy density as

$$
\varepsilon=\frac{\langle E\rangle}{V_{\text {ex }}}=-\frac{1}{V_{\text {ex }}} \frac{\partial}{\partial B} \ln Z\left(B, V_{\text {ex }}, \lambda\right)=\frac{\dot{\Delta}}{V_{\text {ex }}} \varepsilon_{p t} .
$$

Inserting eq (2.22) into eq (2.28) and solving for $\varepsilon$ we find:

$$
\varepsilon(B, \lambda)=\frac{\varepsilon_{p t}(B, \lambda)}{1+\varepsilon_{p t}(B, \lambda) / 4 B} .
$$

Hence we can write eq (2.22) also in another form:

$$
V_{\text {ex }}=\Delta\left(1+\varepsilon_{p t}(B, \lambda) / 4 B\right) .
$$


Using eq (2.30) we find for the baryon density, pressure, and entropy density:

$$
\begin{aligned}
& v=\frac{\nu_{p t}}{1+\varepsilon_{p t} / 4 B} \\
& p=\frac{p_{p t}}{1+\varepsilon_{p t} / 4 B} \\
& s=\frac{s_{p t}}{1+\varepsilon_{p t} / 4 B} .
\end{aligned}
$$

We now have a complete set of equations of state for the observable quantities as functions of the chemical potential $\mu$, the temperature $T$, and the external volume $V_{\text {ex }}$. While these equations are semi-analytic, one has to evaluate the different quantities numerically owing to the implicit definition of $\phi(B, \lambda)$ that determines $\ell n Z$. However, when $B, \lambda$ approach the critical curve, figure 2.?, we easily find from the singularity of $\phi$ that $\varepsilon_{\mathrm{pt}}$ diverges and therefore

$$
\begin{aligned}
& \varepsilon \longrightarrow 4 B \\
& p \longrightarrow 0 \\
& \Delta \longrightarrow 0
\end{aligned}
$$

These limits indicate that at the critical line matter has lumped into one large cluster with the energy density $4 B$. No free volume is left and as only one cluster is present the pressure has vanished. However, the baryon density varies along the critical curve; it falls with increasing temperature. This is easily understood: as the temperature is increased more mesons are produced that take up some of the available space. Therefore hadronic matter can saturate at lower baryon density. We further note here that in order to properly understand the apoproach to the phase boundary one has to incorporate and understand the properties of the hadronic world beyond the critical curve. Therefore we now turn to the study of the world of quarks and gluons and ultimately of the phase of matter consisting of these quanta.

\section{THE WORLD OF QUARKS AND GLUONS}

From the study of hadronic spectra as well as from hadron-hadron and hadron-lepton interactions there has emerged convincing evidence for the description of hadronic structure in terms of quarks [15]. For many purposes it is entirely satisfactory to consider baryons as bound states of three fractionally charged particles, while mesons are quark-antiquark bound states. One of the central aims of this and the next section is to show how this picture of hadrons $c$ an be reconciled with the description of hot hadronic matter consisting of individual particles described in the preceeding section. 
We now recall some fundamental assumptions about the strong interactions, as needed here. The elementary quantum fields which appear in quantum chromodynamics are:

Spin 1: gauge bosons - gluons $\quad G_{\mu}^{j}, \quad i=1 \ldots 8$

Spin 1/2: baryonic matter - quarks $q_{\gamma}^{\alpha}, \quad \begin{aligned} & a=R, G, B=c 010 r \\ & \gamma=d, u, s, c, b,(t)=f 1 \text { avor }\end{aligned}$

The octet of gauge bosons $G_{\mu}$ mediates the quark-quark and - antiquark interactions between the color triplets \{Red, Green, Blue\} and antitriplets. The gauge vector fields are written as

$$
G_{\mu} \equiv \sum_{i=1}^{8} G_{\mu}^{i} \frac{\lambda^{i}}{2}
$$

where $\lambda^{i}$ are the generators of the SU(3) algebra [16]

$$
\left[\lambda^{i}, \lambda^{j}\right]=2 i f^{i j k} \lambda^{k}
$$

Only quarks and antiquarks carry baryon number $( \pm 1 / 3)$. The flavor of quarks represents all internal quantum numbers conserved in strong interactions - the up and down quarks carry $\pm 1 / 2$ units of $I_{z}$ (isospin) and combine to form the Towest $\bar{b}$ aryonic isospin doublet

$$
\left(\begin{array}{l}
p \\
n
\end{array}\right)=\left(\begin{array}{l}
\text { uud } \\
\text { ddu }
\end{array}\right)
$$

and the mesonic isospin triplet

$$
\left(\begin{array}{c}
\pi^{+} \\
\pi^{0} \\
\pi^{-}
\end{array}\right)=\left(\begin{array}{c}
u \bar{d} \\
\frac{1}{\sqrt{2}}(-u \bar{u}+d \bar{d}) \\
\bar{u} d
\end{array}\right) \text {. }
$$


These were the input particles of the statistical bootstrap model. The heavier flavors of quarks include the strange, charm, bottom and perhaps the as yet undiscovered top quark. The electric chärge of $u, c, t$ is $+2 / 3$ and that of $d, s, b$ is -173 .

It is the color-charge of the flavored quarks that introduces the quarkquark interactions. The important fact is that all known hadrons are color neutral (i.e., color singlets). Including color into the wave functions eq (3.3) and ignoring the space and spin degrees of freedom we have, $\underline{e} . \underline{g}$. ,

$$
\begin{gathered}
p=\frac{1}{\sqrt{6}}\left(u^{R} u^{G} d^{B}-u^{G} u^{R} d^{B}+u^{G} u^{B} d^{R}-u^{B} u^{G} d^{R}+u^{B} u^{R} d^{G}-u^{R} u^{B} d^{G}\right) \\
\pi^{+}=\frac{1}{\sqrt{3}}\left(u^{R-R}+u^{G-G}+u^{B-B}\right)
\end{gathered}
$$

where the $p$, and baryons in general, are color-antisymmetric and $\pi$ is color symmetric. The antisymmetry of baryonic wave functions in a hidden degree of freedom has been one of the original reasons for the introduction of color. otherwise, e.g., $\left(\Delta^{++}\right) I=3 / 2=($ uuu $) I=3 / 2$ could not have an ant isymmetric quark wave Fuñction as required for Fermions. Further experimental evidence [17] of color includes the $\pi^{\circ} \rightarrow 2 \gamma$ decay rate and the size of the $e^{+} e^{-} \rightarrow$ hadrons annihilation cross section. However, the evidence for color as a dynamical degree of freedom, in particular, as being responsible for quarkquark interactions, is derived from deep-inelastic lepton-nucleon scattering, from a detailed study of $\mathrm{e}^{+} \mathrm{e}^{-}$annihilation into hadrons, and in particular, from the flavor independence of the charmonium and upsilonium potential with quantitative agreement between the experimental and the theoretical excitation spectra.

The Lagrangian of quarks and gluons is very similar to that of electrons and photons

$$
L_{Q E D}=\bar{\psi}(\gamma \cdot(p-e A)-m) \psi-\frac{1}{4} F_{\mu \nu} F^{\mu \nu}
$$

except for the required summations over flavor and color:

$$
\begin{aligned}
L_{Q C D} & =\sum_{r}^{f 1 \text { avors }}\left(\sum_{\alpha=1}^{3} q_{r}^{\alpha}\left(\gamma \cdot p-m_{r}\right) q_{r}^{\alpha}+g \sum_{\alpha, \beta=1}^{3} \bar{q}_{r}^{\alpha} \gamma^{\mu} \sum_{i=1}^{8}\left(\frac{\lambda^{i}(\alpha \beta)}{2} G_{\mu}^{i}\right) q_{r}^{\beta}\right) \\
& -\frac{1}{4} \sum_{\alpha=1}^{8} F_{\mu \nu}^{i} F^{\mu \nu}+\text { herm. conj. + gauge fixing }
\end{aligned}
$$


The flavor dependent masses $m_{r}$ of the quarks are small. For $u, d$ flavors one estimates $m_{u, d} \sim 5-20 \mathrm{MeV}$ when the strange quark mass is chosen in the range 150-280 MeV. "In particular [18],

$$
\begin{aligned}
& \frac{m_{d}-m_{u}}{m_{d}+m_{u}} \approx \frac{1}{3} ; \\
& \frac{m_{u}}{m_{d}}=0.38 \pm .13 ; \\
& \frac{m_{d}}{m_{s}}=0.045 \pm 0.011 ;
\end{aligned}
$$

The heavy quark mass differences can be obtained reliably from the detailed study of quarkonium spectra [19], [20]

$$
m_{b}-m_{c}=3400 \mathrm{MeV} ; m_{c}-m_{s}=1280 \mathrm{MeV} \text {. }
$$

The color field strengths are now

$$
F_{\mu \nu}^{j}=\partial_{\mu} G_{\nu}^{j}-\partial_{\nu} G_{\mu}^{j}+g f^{i j k} G_{\mu}^{j} G_{\nu}^{k} \text {. }
$$

We record the nonlinearity of $F$, which is required to secure the invariance under local non-abelian gauge transformations. The presence of this glue-glue interaction leads to major differences between the properties of QED and QCD. As an example let us consider briefly the asymptotic freedom of gauge theories [21].

To introduce the subject we note that it is often convenient to define a q-dependent coupling constant, $\underline{e} . \underline{g} .$, through

$$
\frac{e^{2}}{4 \pi} D\left(q^{2}\right)=-\alpha\left(q^{2}\right) \frac{1}{q^{2}}
$$


where $D$ in this case is the QED longitudinal photon propagator, ignoring for the moment the transveral photon degrees of freedom. In terms of the polarization function $\pi\left(q^{2}\right)$ we have

$$
\alpha\left(q^{2}\right)=\frac{e^{2} / 4 \pi}{1+\pi\left(q^{2}\right)}=\left\{\begin{array}{l}
\frac{e^{2}}{4 \pi}=\frac{1}{137}, q^{2}+0 \\
\frac{\alpha(0)}{1-\alpha(0) \frac{1}{6 \pi} \ln \left(-\frac{q^{2}}{m_{e}^{2}}\right)}, q^{2}>m_{e}^{2}
\end{array}\right.
$$

or equivalently with the more complete form of the polarization function

$$
\begin{aligned}
\alpha^{-1}\left(q^{2}\right) & =\alpha^{-1}(0)+\Pi\left(q^{2}\right) / \alpha(0) \\
& =\alpha^{-1}(0)-\frac{q^{2}}{6 \pi} \int_{0}^{\infty} \frac{d M^{2}}{M^{2}} \frac{\left(1+\frac{2 m^{2}}{M^{2}}\right) \sqrt{1-\frac{4 m^{2}}{M^{2}}}}{M^{2}-q^{2}}
\end{aligned}
$$

The electron-loop polarization function $\Pi\left(q^{2}\right)$ follows from the iteration of the standard lowest order diagram:

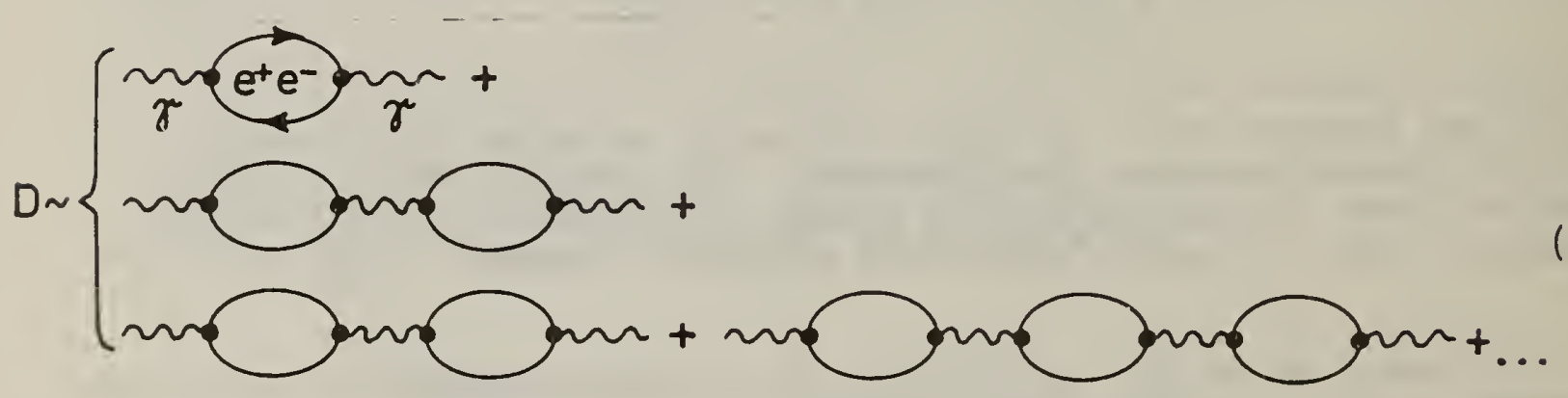

As easily can be seen, $\alpha^{-1}\left(q^{2}\right)$ decreases with increasing $q^{2}>4 m e^{2}$. This means that for short distances the effective strength of the QED interaction increases. Only because of the magnitude of $\alpha^{-1}(0)=137$ is this effect usually unimportant. However, it has been quite precisely measured. 
In QCD additional contributions originate in the glue-glue interaction;

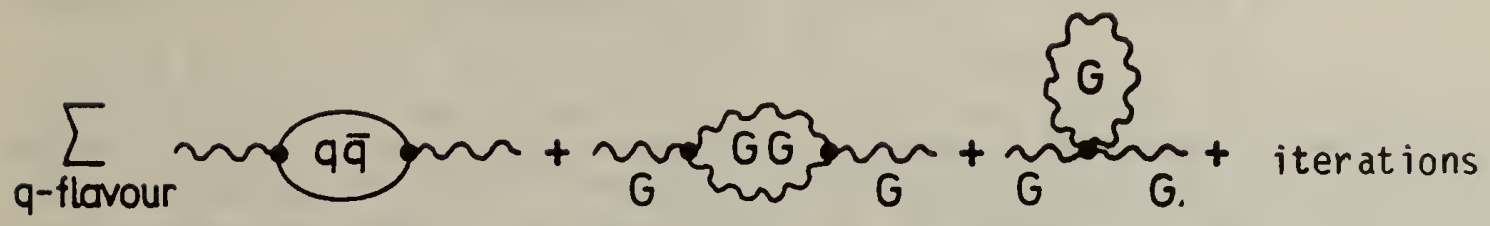

Since gluons are massless we cannot select the point $q^{2}=0$ as a reference point. We have [21],

$$
\alpha_{S}^{-1}\left(q^{2}\right)=\alpha_{S}^{-1}\left(-\mu^{2}\right)+\frac{1}{4 \pi}\left[11-\frac{2 n q}{3}\right] \text { ln } \frac{-q^{2}}{\mu^{2}}
$$

with a certain space-like $q^{2}=-\mu^{2} \neq 0$ serving now as reference. $n_{q}$ is the number of light quark flavors $\left(\mathrm{m}^{2} r<\left|q^{2}\right|\right)$. For large $q^{2}$, absorbing the first term on the right hand side in eq (3.16) in the definition of $\mu^{2}$ we have the so called asympototic freedom formula:

$$
\alpha_{S}\left(q^{2}\right)=\frac{12 \pi}{33-2 n_{q}} \frac{1}{\ln \left(-q^{2} / n^{2}\right)}
$$

which, unlike the case of QED leads to falling $\alpha_{s}$ with rising $\left|q^{2}\right|$ for the likely case $n_{q} \leqslant 16$. Hence, at asymptot ically short distances the interaction diminishes and the theory becomes free. We emphasize that therefore the chain of approximations leading to eq (3.17) here, i.e., in QCD, becomes more and more consistent. In figure 3.1 the running coupling constant is shown for space-like, $q^{2}<0$ and time-like $q^{2}>0$, momenta. In the latter case we show $\operatorname{Re} \alpha_{s}$ :

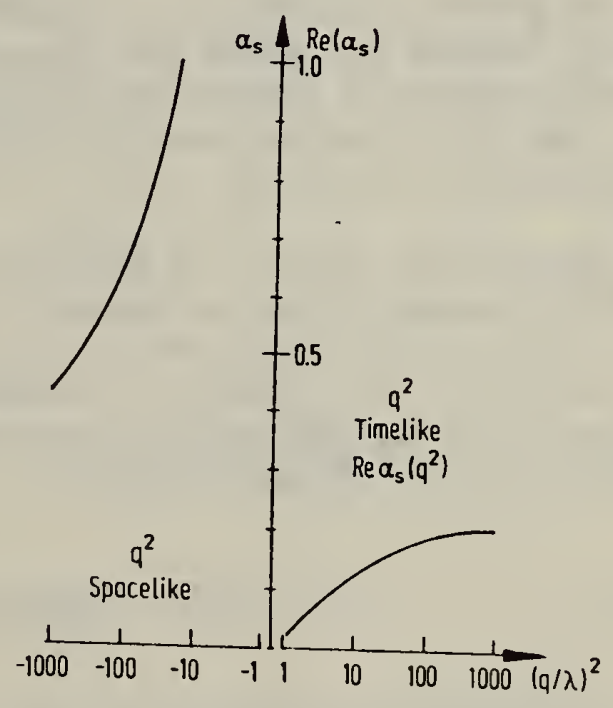

Fig. $3.1 \alpha\left(q^{2}\right)$ for space-like and $\operatorname{Re}\left(\alpha\left(q^{2}\right)\right.$ for time-like momenta. 


$$
\operatorname{Re} \alpha_{s}\left(q^{2}>0\right)=\frac{12 \pi}{33-2 n_{q}} \frac{\ln \left|q^{2} / \Lambda^{2}\right|}{\left(\ln \left|q^{2} / \Lambda^{2}\right|\right)^{2}+\pi^{2}}
$$

We notice that at the presently accessible momenta, i.e., up to $100 \Lambda(\Lambda \sim 200-$ $400 \mathrm{MeV}), \alpha_{s}$ is considerably smaller for time-like $q^{z}$ than for space-like $q^{2}$. For large $\alpha_{s}\left(q^{2}\right)$ other than order $g^{2}$ diagrams must be included into the determination of $\alpha_{s}\left(q^{2}\right)$. This may change the value of $\Lambda$ which at this stage is a phenomenological parameter fitted to the experiment and which reflects in its value the order of the expansion. At present the actual value of $\Lambda$ is rather uncertain since as can be seen in eq (3.17) only small logarithmic corrections are involved. Quarkonium fits (space-like $q^{2}$ ) favor $\Lambda \sim 400 \mathrm{MeV}$ [19] while deep-inelastic experiments (time-like $q^{2}$ ) lead to $\Lambda=100 \pm 100 \mathrm{MeV}$ [17].

As we have seen above, the strength of the glue-glue interaction influences significantly the glue propagation in the (perturbative)vacuum. Little is known about the behavior of the glue propagator, i.e., $\alpha\left(q^{2}\right)$, at small $q^{2}$, that is at large distances. Attractive channels in the glue-glue interaction are expected to induce a gluonic structure onto the vacuum state [22],[23]. To appreciate this remark let us imagine a box of size $R$ filled with a gas of $N$ gluons. Including a $1 / R$ kinetic energy and an attractive long range interaction we have for the energy density E/V:

$$
\varepsilon_{B o x}(N) \sim N / R^{4}-N^{2} g^{2} / R^{4}
$$

and hence for some $N=N_{C}$ it would cost no energy to fill the box with gluons. Hence the empty ${ }^{c}$ box (perturbative vacuum) and the box with $\mathrm{N}_{\mathrm{cr}}$ particles would be degenerate. We conclude that an improved gound state, i.e., the true vacuum, has to be constructed. Such a state would have a lower energy density than the value of the perturbative state.

The energy density of the perturbative state is defined with respect to the true vacuum state and hence is by definition a positive quantity, denoted by $B$. This notion has been introduced originally in the MIT bag model [10], but initially in a different context. The value of $B$ is derived phenomenologically from a fit to the hadronic spectrum [10],[24] or from sum rules considerations [25] which give

$$
B=[(140-210) \mathrm{MeV}]^{4}=(50-250) \mathrm{MeV} / \mathrm{fm}^{3} .
$$

The central assumption of the quark-bag approach is that inside a hadron where quarks are found the true vacuum structure is displaced or destroyed. One can turn this point around: quarks can only propagate in domains of space in which the true vacuum structure is absent. This statement is a resolution of the quark confinement problem. The remaining difficult problem is to show the incompatibility of quarks with the true vacuum structure. Examples of 
such behavior in ordinary physics are easily found: e.g., a light wave is reflected from a mirror surface; magnetic field lines are expelled from superconductors; etc.

In this spirit we may argue that all color-charged particles are reflected at the true vacuum surface (stationary waves) or alternatively, may under certain circumstances deform the surface. Whatever is the case, the presence of color electric fields in a volume element is incompatible with the presence of the vacuum structure. It is interesting to note that the Lorentz covariance of the theory requires that a negative pressure $p=-B$ as seen from the perturbative vacuum acts on the surface between the true and the perturbative vacuum. Hence, in the absence of other forces the excited space domain containing the perturbative vacuum would quickly vanish.

In this picture of hadronic structure and quark confinement all colorless assemblies of quarks, antiquarks, and gluons can form stationary states, called a quark bag. In particular all higher combinations of the three-quark baryons (qqq) and quark-antiquark mesons (qव) form a permitted state, i.e., a hadronic resonance, much in the spirit of the statistical bootstrap modeT of section 2 .

The energy of a hadronic bag of radius $R$ including the particle and the volume bag terms is:

$$
E(R)=\left(\sum_{i} X_{i}\right) / R+\frac{4}{3} \pi R^{3} B
$$

where $X_{j} / R$ are the appropriate eigenvalues, $i . e .$, single particle energies of "confined" particles and the sum is over ail quanta in the bag. Effects of interactions can be considered to be included in $x_{i}$, in which case the $x_{i}$ become functions of the interaction strength and the number of particies present. For massive particles an additional dependence on $\mathrm{mR}$ is recorded. The radial pressure (force/area) on the surface is:

$$
P_{r}=\frac{-\partial E / \partial R}{4 \pi R^{2}}=-B+\frac{\left(\Sigma X_{j}\right)}{4 \pi R^{4}}
$$

which, combined with eq (3.21) leads to the interesting relation

$$
E(R)=\left(3 P_{r}+4 B\right) V
$$

For a radially stable object $P_{r}$ must vanish, or, in other words, $E(R)$ must have a minimum. From eq (3.22) we have

$$
R_{\min }=B^{-1 / 4}\left(\frac{\sum X_{i}}{4 \pi}\right)^{1 / 4}
$$


and from eq (3.23)

$$
E\left(R_{\min }\right)=4 B V=B^{1 / 4}\left(\sum X_{j}\right)^{3 / 4} \frac{4}{3}(4 \pi)^{1 / 4}
$$

From eqs (3.24), (3.25) we learn that the radius of the bag grows with $\left(\Sigma X_{j}\right)^{1 / 4}$, while it decreases as $B^{-1 / 4}$, as could be expected from dimensional arguments. Similarly, the energy (mass) of the bag grows with $\left(\Sigma X_{j}\right)^{3 / 4}$, but also with $B^{1 / 4}$, as expected on dimensional grounds. The remarkable relation $E=4 B V$ is often called the virial relation as it follows from the dimensionality of space alone. We further notice that the dimensionless structure constant

$$
R_{\min } E\left(R_{\min }\right)=\frac{4}{3}\left(\Sigma x_{i}\right)
$$

can not be directly compared with the values known for example for protons:

$$
R_{\text {proton }}^{\text {charged }} M_{\text {proton }}=3.82
$$

since $R_{\min }$ is not the charge radius but the hadronic radius of the bag. To illustrate the difference between both quantities, assume that the wave function of the quarks inside of the bag is approximately given by

$$
\phi_{a_{j}} \sim N \cos \left(x_{i} \frac{r}{R}\right)
$$

with $x_{0}=\pi / 2$ for the lowest node-less state such that $\phi_{q}(r=R)=0$. The charge radius is

$$
\left(R^{\text {charge }}\right)^{2}=\frac{\int_{0}^{R} r^{2} \cos ^{2}\left(\frac{\pi}{2} \frac{r}{R}\right) d^{n} r}{\int_{0}^{R} \cos ^{2}\left(\frac{\pi}{2} \frac{r}{R}\right) d^{n} r}
$$

where $n=3$ is the space dimensionality. For consistency with the wave function (3.28) we choose $n=1$ and find 


$$
R^{\text {charge }} / R=\left(\frac{1}{3}-\frac{2}{\pi^{2}}\right)^{1 / 2}=0.36
$$

In three dimensions the difference between the charge radius and hadronic radius is not so pronounced, but the latter is expected to be $40-50 \%$ larger than the charge radius. Returning to eq $(3.26)$ we note that using $X_{0}=\pi / 2$ one finds for three quarks

$$
R_{\min } E\left(R_{\min }\right)=2 \pi
$$

which is about twice the anticipated charge radius size, eq (3.27), and in particular leads to a much too large hadronic radius $(\sim 1.3 \mathrm{fm})$.

The true lowest eigenvalue $x_{0}$ omitting for the present the interactions is found solving the three-dimensional Dirac equation with the bag boundary condition [10], which leads to

$$
R E=X_{0}=2.04
$$

When inserted into eq (3.26), we would find for three quarks for the hadronic radius of a nucleon

$$
R_{\min } \sim 4 \times 2.04 \times 197 \mathrm{MeV} \mathrm{fm} / 940 \mathrm{MeV} \cong 1.7 \mathrm{fm}
$$

which clearly is an unacceptable result. Obviously, something is missing in eq (3.22), and it must be added in order for it to give the proper phenomenology of hadronic states. In the original MIT bag approach an additional zero-point energy

$$
E_{0} \approx \frac{-Z_{0}}{R}
$$

was introduced. This can be taken care of by replacing $\left(\Sigma X_{i}\right)$ in above formula by $\left(\Sigma x_{i}-z_{0}\right)$.

With this we find for the proton

$$
R_{\min }=\frac{4}{3}\left(\Sigma x_{i}-z_{0}\right) / m_{p}
$$

which requires $Z_{0} \sim 2$ in order to make $R_{\text {min }}$ sufficiently small, $i_{. e} .,<1 \mathrm{fm}$, as long as the noninteracting value $X_{0}=2.84$ is employed. The constraint arising from the fact that the sum of the bag energy and $E_{0}$ must not become negative has been so far little appreciated. Namely, a negative value is 
unacceptable, as it leads to stable empty bags which would contradict the characteristics of the true vacuum. Using the virial relation eq (3.23), this requirement becomes:

$$
0<E_{0}+B V=E_{0}+\frac{1}{4} m_{p}=\left(\frac{1}{4} m_{p} R_{\min }-Z_{0}\right) \frac{1}{R_{\min }} .
$$

Recalling now eq (3.35) we find from eq (3.36) that we must have

$$
z_{0}<\frac{1}{4} \Sigma x_{i}
$$

This consideration is equally valid for mesons, but is less conclusive since other effects intervene, such as restoration of translational invariance to the quark bound states. For nucleons, eq (3.37) implies

$$
z_{0}<\frac{3}{4} x_{0}
$$

which is usually just barely satisfied once one includes the interactions. For free quarks the introduction of $z_{0}$ coupled with the constraint (3.36) reduces the numerical value eq $(3.33)$ at most by a factor $3 / 4$, which is not enough to yield the empirical value.

Clearly, this discussion shows the necessity to include the quark-quark interaction and eventually project on translationally invariant states in order to resolve this apparent contradiction. For our present discussion it is important to realize that the quark-bag picture can be made internally consistent only when the quarks are allowed to interact. Unfortunately, for "small" bags, i.e., for normat hadronic states, this opens the Pandora's box of all complex self-energy, exchange and other contributions leading to the large current confusion in the field of how such large corrections can mutually cancel; we do not discuss such problems as the theoretically infinite values for $Z_{0}$, or the influence of pionic degrees of freedom when the bag radius is too small. However, we note that most of these problems disappear in large bags, i.e.., those containing many single-particle excitations.

\section{FROM QUARK BAG TO QUARK-GLUON PLASMA}

A large quark-gluon bag, i.e., one which contains many particles, is characterized by the available modes $x_{j}$ and their occupation numbers $n_{j}$. An important simplification of its description arises if it is possible to use a statistical treatment.

As the $u$ and $d$ quarks are almost massless inside a bag they can be produced in pairs, and at moderate internal excitations, i.e., temperatures, many qव pairs will be present. Similarly, ss pairs also wiTl be produced. We will 
return to this point at length below. Furthermore, real gluons can be excited and will be included here in our considerations. We now first convince

ourselves that already a moderate number of quarks justifies the statistical approach. For the degenerate Fermi gas of quarks the number of light quarks $(u$ and $d)$ determines the quark Fermi energy $\mu_{q}$. Omitting for the present the qq interactions we have

$$
3 b=N_{q}=2 \times 2_{f} \times 3 c \times V \frac{4 \pi}{(2 \pi)^{3}} \frac{1}{3} \mu_{q}^{3}=V \frac{2}{\pi^{2}} \mu_{q}^{3}
$$

where the indices $s, f, c$ refer to spin, flavor, and color degeneracies resper:tively. Equation (4.1) establishes a relation between a given baryon number $b$ (quarks carry $1 / 3$ unit of baryon number) and the variables $V$ (volume) and $\mu_{q}$. The energy of the quark bag is easily obtained noting that

$$
E_{q, g a s}=2 s \times 2_{f} \times 3_{c} \times V \quad \int \frac{d^{3} p}{(2 \pi)^{3}} \sqrt{m_{q}^{2}+p^{2}} \theta\left(\mu^{2}-\left(p^{2}-m_{q}^{2}\right)\right)
$$

Hence in the limit of small quark mass, i.e., $\mu_{g} \gg m_{g}$, we find, omitting here agdin for the sake of simplicity the qq-interaction term,

$$
E\left(V, \mu\left(N_{q}, V\right)\right)=B V+V \frac{3}{2 \pi^{2}} \mu_{q}^{4}+O\left(m_{q} / \mu\right)
$$

In order to determine the explicit dependence on a given quark number (baryon number) we use eq (4.1) to eliminate $\mu_{q}$ :

$$
E\left(V, N_{q}\right)=B V+\frac{N_{q}^{4 / 3}}{V^{1 / 3}} \frac{3}{4}\left(\frac{\pi^{2}}{2}\right)^{1 / 3} .
$$

This expression has as before a minimum as function of the volume $V$, which corresponds to the equilibrium state:

$$
P=-\left.\frac{\partial E}{\partial V}\right|_{S, b} \stackrel{!}{=} 0=-B+\frac{1}{4}\left(\frac{\pi^{2}}{2}\right)^{1 / 3}\left(\frac{N_{q}}{V_{\text {min }}}\right)^{4 / 3} .
$$

Combining eqs (4.4) and (4.5), of course we find again

$$
E_{\min }=\left.4 B V\right|_{\min }
$$


and hence we see that the energy density is 48 also in the statistical bag (virial theorem). Combining eq (4.4) with (4.5), we find furthermore for the energy per quark the usual result:

$$
\left(E / N_{q}\right)_{\min }^{g a S}=\left(\mu_{q}\right)_{\min }=B^{1 / 4}\left(2 \pi^{2}\right)^{1 / 4}=2.11 B^{1 / 4} .
$$

Here $\left(\mu_{q}\right)_{\text {min }}$ is the chemical potential that is found inserting eq (4.5) into eq (4.1), upon which the $N_{q}$-dependence drops out. This result, eq (4.7) can be compared with a similar result for the smallest closed-shell bag which contains 12 quarks owing to ${ }_{s} \times 2_{f} \times 3_{c}=12$. With $x_{0}=2.04$ we find from eq $(3.25)$

$$
(E / 12)_{b a g}=\frac{(12 \times 2.04)^{3 / 4}}{12} \frac{3}{4}(4 \pi)^{1 / 4} B^{1 / 4}=2.3 \mathrm{~B}^{1 / 4} .
$$

Thus we conclude that the statistical result, eq (4.6) is in a remarkably good agreement with a closed shell bag even when its baryon number is only 4 . As the energy per quark in the statistical bag approach is slightly underestimated we conclude that the quark (i.e., baryon) density

$$
N_{q} / V=(E / V)\left(N_{q} / E\right)=4 B /\left(E / N_{q}\right)
$$

is somewhat overestimated.

As a final remark we note that eqs (4.3) and (4.6) imply that the energy per baryon in the bag is just $\mu$, $\underline{i} . \underline{e}$, the baryon chemical potential

$$
E / b=\frac{4}{3} E_{q} /\left(N_{q} / 3\right)=3 \mu_{q}=\mu
$$

The factor 3 is necessary to account for the baryon number $1 / 3$ of the quarks: three quarks form one baryon. We note that from eq (4.10) stems the conventional wisdom that $\mu_{q}=m_{p} / 3$ at $T=0$. Omitting the bag term in eq (4.2) one finds the well known relativistic ideal-gas result

$$
E_{q} / N=3 / 4 \mu
$$

Thus we see that the bag term is a necessary ingredient for recovering the hadronic gas limit [9]

$$
E /\left.b\right|_{T=0}=\mu
$$

where $T$ is the temperature. 
Quarks will not always form a degenerate Fermi gas, especially inside a large bag. Depending on the creation history of the bag it is very likely that in an initial stage some of the quarks will be in excited states. In the statistical approach this situation easily can be described by introducing a quark temperature $T=1 / B$ which describes the internal excitations of each bag (= hadronic cluster) [26]. This does not imply an internal thermodynamic equilibrium of the quark gas in the bag. However, an assembly of excited bags in mutual thermal contact that is sharing a certain internal excitations, may be already similar in nature to the Gibb's ensemble, i.e., an infinity of interacting identical subsystems.

Hence, though each individual bag may be far from thermodynamic equilibrium, an assembly of bags able to scatter several times may be much closer to it. When making these remarks here we have particularly in mind highly excited nuclear matter as created in relativistic nuclear collisions, and, perhaps in antiproton annihilations on nuclei. Other circumstances prevail in $\mathrm{e}^{+} \mathrm{e}^{-} \rightarrow$ hadrons or even $\mathrm{pp}$ reactions. But also in our case the word "kinetic equilibrium" has to be used with great care: the further the mean kinetic energy of quarks is from $\sim T$, the less reliable becomes a priori the equilibrium assumption. We record here, however, that particle spectra from $p-p$ collisions [8] behave as if a thermal equilibrium were always reached. In thermodynamical models of hadron reactions therefore the concept of "preformed" equilibrium has been introduced.

With these remarks in mind we now turn to the description of excited quark bags with the help of quantum statistical methods. We will initially ignore the effect of quark-quark interactions and return to this problem further below. In principle, we may avoid the formal development and simply proceed by including temperature through a Fermi distribution function in eq (4.2). However, as was outlined in section 2 a complete description of the thermodynamical behavior of a many-particle system can be derived from the grand canonical partition function $Z$. Hence it is more useful for further developments to obtain right-away the grand partition functions for ideal Fermi and Bose gases. We follow here initially the standard textbooks [27] in calculating the grand canonical partition function defined as

$$
\left.Z\left(B, \mu_{q}, \ldots\right)=\operatorname{Tr}\left(e^{-B(\hat{H}}-\mu \hat{Q}\right)\right)
$$

where $H$ is the Hamiltonian of the system and $Q$ is the baryon charge operator. The chemical potential $\mu$ determines the average baryon number of the system. The trace is to be carried out over all allowed states of the many-body system. To appreciate the importance of the quantity (4.12) we note that

$$
\begin{gathered}
\langle Q\rangle \equiv \frac{\operatorname{Tr} Q e^{-\beta(\hat{H}-\mu \hat{Q})}}{\operatorname{Tr} e^{-\beta(\hat{H}-\mu \hat{Q})}}=\frac{1}{\beta} \frac{\partial}{\partial \mu} \ln Z(\beta, \mu, \ldots) \\
\left.\langle H\rangle \equiv \frac{\operatorname{Tr} H e^{-\beta(\hat{H}-\mu \hat{Q})}}{\operatorname{Tr} e^{-\beta(\hat{H}-\mu \hat{Q})}}=-\frac{\partial}{\partial \beta} \ln Z(\beta, \mu, \ldots)+\mu \hat{Q}\right\rangle .
\end{gathered}
$$


The partition function may depend implicitly on other quantities such as the volume or even the shape of the considered quantum system.

In the particle number representation the trace, eq (4.12), can be easily carried out for free quarks. With

$$
\begin{gathered}
\hat{H}=\sum_{i, l} \varepsilon_{i}^{\ell} \hat{n}_{i}^{\ell}+\sum_{i, \ell} \varepsilon_{i}^{\ell-\ell} n_{i}^{\ell} \\
\hat{Q}=\sum_{i, \ell} b_{\ell}\left(\hat{n}_{i}^{\ell}-\hat{n}_{i}^{\ell}\right)
\end{gathered}
$$

where $\hat{n}_{i}^{\ell}$ is the number operator of the $i^{\text {th }}$ single-particle state of a quark ( $\hat{n}_{j}^{\ell}$ for antiquarks) with (discrete) quantum numbers " $\ell$ ", such as flavors. b $\ell$ is the baryon charge $+1 / 3$ for quarks and of opposite sign for antiquarks as introduced already explicifly in eq (4.15b). A quantum state is characterized by occupation numbers $\hat{n}_{j}^{\ell}$, $\hat{n}_{j}^{\ell}$ of quarks and antiquarks. Hence the trace which sums only the diagonal matrix elements is

$$
z_{q}=\sum_{\left\{n_{i}^{\ell}, \bar{n}_{j}^{\ell}\right\}} e^{-\left[\beta \sum_{i, l}\left(\varepsilon_{i}^{\ell}-1 / 3 \mu\right) n_{i}^{\ell}+\sum_{i, l}\left(\varepsilon_{i}^{\ell}+1 / 3 \mu\right) \bar{n}_{i}^{\ell}\right]} .
$$

Here the sum runs over all sets of numbers $n_{i}^{\ell}, n_{j}^{\ell}$. We factorize the partition function in terms of the discrete quantum numbers $\ell$ :

$$
\begin{aligned}
& z_{q}=\pi z_{\ell} \\
& z_{\ell}=\sum_{\left\{n_{i}^{\ell}, n_{i}^{\ell}\right\}} \pi e^{\left.-\beta\left(\varepsilon_{i}^{\ell}-1 / 3 \mu\right) n_{i}^{\ell}-\beta\left(\varepsilon_{i}^{\ell}+1 / 3 \mu\right) n_{i}^{\ell}\right]} .
\end{aligned}
$$

The infinite product over all states can be interchanged with the infinite sum over all occupation numbers leading to:

$$
\ln Z_{\ell}=\sum_{i}\left[\left(\ln \sum_{n_{i}^{\ell}} e^{-\beta\left(\varepsilon_{i}^{\ell}-1 / 3 \mu\right) n_{i}^{\ell}}\right)+\left(\ln \sum_{-n_{i}^{\ell}} e^{-\beta\left(\varepsilon_{i}^{\ell}+1 / 3 \mu\right) n_{i}^{\ell}}\right)\right] .
$$


We can have only $n_{j}^{\ell}=0,1$ for fermions. Hence we find the well known result ( $f=$ flavor, $s=$ spin, $c=$ color $)$

$$
\ln z_{q}=\sum_{f l a v o r} 2 s_{c}^{3}\left[\sum_{i} \ln \left(1+e^{-\beta\left(\varepsilon_{j}^{f}-1 / 3 \mu\right)}\right)+\sum_{i} \ln \left(1+e^{-\beta\left(\varepsilon_{j}^{f}+1 / 3 \mu\right)}\right)\right]
$$

where the spin and color factors count the respective degeneracies. In the cont inuum limit

$$
\begin{aligned}
& \sum_{i} \rightarrow \int \frac{d^{3} x d^{3} p}{(2 \pi)^{3}}=V \int \frac{d^{3} p}{(2 \pi)^{3}} \\
& \varepsilon_{i}^{f} \rightarrow \sqrt{p^{2}+m_{f}^{2}}
\end{aligned}
$$

we find

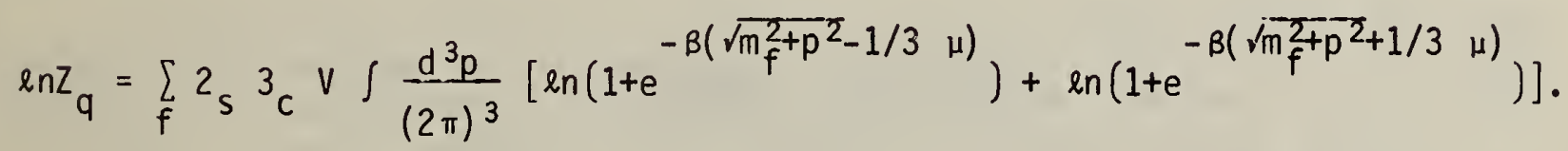

For the light $u$ and $d$ quarks, for which usually $m_{f}<<\mu$ is fulfilled, we can evaluate the momentum integrals analytically [2b], [28]:

$$
\left.(T \quad \operatorname{lnz})_{q}\right)_{\text {light flavors }}=\frac{{ }^{2} \times 2 f_{f} \times 3 \times V}{24 \pi^{2}}\left(\mu_{q}^{4}+2 \mu_{q}^{2}(\pi T)^{2}+\frac{7}{15}(\pi T)^{4}\right)
$$

where we have used the quark chemical potential

$$
\mu_{q}=\frac{1}{3} \mu
$$

which controls the quark number $\mathrm{N}_{\mathrm{q}}-\mathrm{N}_{\overline{\mathrm{q}}} \cdot$

In order to recover the limit $T \rightarrow 0$, eq (4.3), we must introduce a phenomenological bag, i....., vacuum term

$$
\operatorname{lnZ_{\text {bag}}} \equiv-B V B
$$


With eq (4.24) and

$$
\ln z=\ln z_{q}+\ln z_{v a c}
$$

we indeed find

$$
\begin{aligned}
E & =\langle H\rangle=-\frac{\partial}{\partial \beta} \ln Z+\mu \frac{\partial}{\partial \mu} T \ln Z \\
& =B V+\frac{3}{2 \pi^{2}} V\left[\mu_{\mathrm{q}}^{4}+2 \mu_{\mathrm{q}}^{2}(\pi T)^{2}+\frac{7}{15}(\pi T)^{4}\right]
\end{aligned}
$$

and at the same time for the baryon number

$$
\begin{aligned}
b / 3 & =\left\langle\frac{1}{3} Q\right\rangle=N_{q}-N_{\bar{q}}=\frac{\partial}{\partial \mu_{q}} T \ln Z \\
& =\frac{2}{\pi^{2}} V\left(\mu_{q}^{3}+\mu_{q}(\pi T)^{2}\right)
\end{aligned}
$$

Equations (4.26) and (4.27) generalize the $T=0$ results, eq (4.1) and (4.3) to finite temperatures. We note also that for finite $T$ it is possible to el iminate $\mu_{q}$ analytically from eq $(4.26)$ with the help of Cardan's formulae [28] for eq $(4.27)$, and to obtain $E(V, b, T)$.

We have not yet considered one quite important aspect of the excited bag, viz., the possible presence of real transverse gluons. At present the evidence for the existence of perturbative gluons inside bags is not quite conclusive. Some theoretical calculations [29] indicate that glue could be admixed to the quark wave functions in bags. However, gluonium (glue-only bags) have not been conclusively established experimentally [30]. We record here that the color confining bag boundary condition should be different for perturbative gluons from that postulated for the perturbative quarks, as a direct consequence of the fact that the vacuum structure originates in the gauge (glue) sector of QCD. While this problem remains to be resolved it is very likely that in a large quark bag at finite temperature these transverse gluons will be present with an abundance corresponding to that expected from the blackbody radiation law, i.e., as given by the Stefan-Boltzmann equation. To include these bosonic degreess of freedom we evaluate eq (4.16) taking into account the fact that the occupation number of glue modes can be $n_{i}=1,2, \ldots \infty$, and that they do not carry baryon charge. We thus find 


$$
z_{g}=\pi \prod_{\ell} \sum_{n=0}^{\infty} e^{-\beta n \varepsilon_{i}^{\ell}}=\prod_{\ell i} \prod_{1-e^{-\beta \varepsilon_{i}^{\ell}}} \text {. }
$$

Here $\ell$ counts the $N^{2}-1=8$ color degrees of freedom as well as the two transverse polarizations. All gluon single-particle energies are degenerate with respect to $\ell$. Taking the continuum limit we find:

$$
\ln Z_{g}=-8 c^{2} s \vee \int \frac{d^{3} p}{(2 \pi)^{3}} \ln \left(1-e^{-\beta|\vec{p}|}\right) .
$$

This expression is very well known and corresponds up to the color factor to the standard photon result. We find explicitly

$$
T \ln Z_{g}=8 \frac{V}{45 \pi^{2}}(\pi T)^{4}
$$

As emphasized at the end of the last section a further effect that must be taken into account is the quark-quark interaction. We shall use here the first order contributions in the QCD running coupling constant $\alpha_{S}\left(q^{2}\right)=g^{2} / 4 \pi$. As $\alpha_{s}\left(q^{2}\right)$ increases when the average moment um exchanged between quarks decreases it would seem that this approach will have only limited validity at relatively low densities and/or temperatures. However, the collective screening and phonon modes in the plasma are of comparable order of magnitude and cancel each other [31]. The influence of perturbative contributions which are governed by the expansion factor $\delta=4 / 3 \alpha_{s} / \pi \sim 0.15-0.3$ is limited. In other words, since $\delta^{2}<0.02-0.09$ the use of first order perturbation theory may be quite adequate [32]. For the case of the quark-gluon plasma in the perturbative vacuum, one finds for the partition function an analytic expression through first order in $\alpha$ neglecting the quark masses. We obtain for the quark Fermi gas $[2 \mathrm{~b}],[2 \mathrm{~g}],[28]$ :

$$
\ln Z_{q}(\beta, \mu)=\frac{g V}{6 \pi^{2}} \beta^{-3}\left[\left(1-\frac{2 \alpha_{S}}{\pi}\right)\left(\frac{1}{4}(\mu \beta)^{4}+\frac{\pi^{2}}{2}(\mu \beta)^{2}\right)+\left(1-\frac{50}{21} \frac{\alpha_{S}}{\pi}\right) \frac{7 \pi^{4}}{60}\right]
$$

where $g=(2 s+1)(2 I+1) N=12$ counts the number of the components, $1 . e$. , the degeneracy of the quark gas. The glue partition function is similarTy reduced by the interactions:

$$
\ln Z_{g}(\beta, \lambda)=V \frac{8 \pi^{2}}{45} \beta^{-3}\left(1-\frac{15 \alpha}{4 \pi}\right) .
$$


We notice above the second relevant difference from the photon gas: aside from the occurrence of the factor 8 associated with the number of gluons there is the term associated with the glue-glue interaction since gluons carry colorcharge.

Finally, let us recall the true-vacuum term en $Z_{v a c}$, eq (4.24). This leads to the required positive energy density $B$ within the volume occupied by the colored quarks and gluons and to a negative pressure on the surface of this region. At this stage, this term is entirely phenomenological as discussed above. The equations of state for the quark-gluon plasma are easily obtained by differentiating

$$
\operatorname{lnz}=\ln z_{q}+\ln z_{g}+\ln z_{v a c}
$$

with respect to $B, \mu$, and $V$. The energy density, baryon number density, pressure and entropy density of $u, d$ quarks and gluons are respectively, written in terms of the baryonic chemical potential $\mu$ and the temperature $T$

$$
\begin{gathered}
\varepsilon=\frac{6}{\pi^{2}}\left[\left(1-\frac{2 \alpha_{s}}{\pi}\right)\left(\frac{1}{4}\left(\frac{\mu}{3}\right)^{4}+\frac{1}{2}\left(\frac{\mu}{3}\right)^{2}(\pi T)^{2}\right)+\left(1-\frac{50}{21} \frac{\alpha_{S}}{\pi}\right) \frac{7}{60}(\pi T)^{4}\right] \\
+\frac{8}{15 \pi^{2}}(\pi T)^{4}\left(1-\frac{15}{4} \frac{\alpha_{S}}{\pi}\right)+B \\
v=\frac{2}{3 \pi^{2}}\left[\left(1-\frac{2 \alpha_{S}}{\pi}\right)\left(\left(\frac{\mu}{3}\right)^{3}+\frac{\mu}{3}(\pi T)^{2}\right)\right. \\
P=\frac{1}{3}(\varepsilon-4 B)
\end{gathered}
$$

$S=\frac{2}{\pi}\left(1-\frac{2 \alpha}{\pi}\right)\left(\frac{\mu}{3}\right)^{2}(\pi T)+\frac{14}{15 \pi}\left(1-\frac{50}{21} \frac{\alpha_{S}}{\pi}\right)(\pi T)^{3}+\frac{32}{45 \pi}\left(1-\frac{15}{4} \frac{\alpha}{\pi}\right)(\pi T)^{3}$.

In eqs (4.34) and (4.37) the second $T^{4}$ and $T^{3}$ terms originate in the gluonic degrees of freedom. In eq (4.36) we have right away used the relativistic relation between the quark and gluon energy density and pressure

$$
P_{q}=\frac{1}{3} \varepsilon_{q}, \quad p_{g}=\frac{1}{3} \varepsilon_{g}
$$

in order to derive this simple form of the equation-of-state. This simple equation-of-state of the quark-gluon plasma is slightly modified when the finite quark masses are considered, or when the QCD coupling constant $\alpha_{s}$ is dependent on the dimensional parameter $\Lambda$. 
As we have already seen in the discussion of the hadronic bag structure in section 3, an assembly of quarks will assume a geometric configuration such as to make the total energy $E(V, b, S)$ as small as possible at fixed given baryon number and fixed total entropy. As is apparent from the first law of thermodynamics

$$
d E=-P d V+T d S+\mu d b
$$

we have

$$
P=-\frac{\partial E\left(V_{2}, \underline{,}, S\right)}{\partial V}
$$

Hence, the geometrically stable configuration $\partial E / \partial V=0$ corresponds also to the configuration with vanishing pressure $P$. Rather than to work in the microcanonical ensemble with fixed $b$ and $S$, we exploit the advantages of the grand cononical ensemble and consider $P$ as a function of $\mu$ and $T$ :

$$
P=\frac{\partial}{\partial V}(T \ln Z(\mu, T, V))
$$

with the result as given by eq (4.36). From eq (4.36) it follows that when the pressure vanishes in a static configuration the energy density is $4 \mathrm{~B}$, independent of the values of $\mu$ and $T$ which fix the line $P=0$. We recall that this has been precisely the kind of behavior found for the hadronic gas. For $P>0$ we have $\varepsilon>4 B$. We recall that in the hadronic gas we always had $\varepsilon \leqslant 4 B$. Thus, in this domain $P>0$ of the $\mu-T$ plane we have the quark-gluon plasma exposed to an external force.

In order to obtain an idea of the form and location of the $P=0$ configuration in the $\mu-T$ plane for the quark-gluon plasma, we rewrite eq (4.36) for $P=0$ :

$$
B=\frac{\left(1-\frac{2 \alpha_{S}}{\pi}\right)}{162 \pi^{2}}\left[\mu^{2}+(3 \pi T)^{2}\right]^{2}-\frac{T^{4} \pi^{2}}{45}\left[\left(1-\frac{5 \alpha_{S}}{3 \pi}\right) 12-\left(1-\frac{15 \alpha_{S}}{4 \pi}\right) 8\right] .
$$

Here, the last term is the contribution of the glue to the pressure. We find that the greatest lower bound on the temperature $T_{q}$ at $\mu=0$ is about $\left(\alpha_{s}=1 / 2\right)$

$$
\mathrm{T}_{\mathrm{q}} \approx .83 \mathrm{~B}^{1 / 4} \sim 160 \mathrm{MeV} \approx \mathrm{T}_{0}
$$


This result shows the expected order of magnitude. The most remarkable point is that it leads to a numerically similar value as that found in the study of the hadronic gas of section 2. Another point worth mentioning is the influence of the strange quarks: they increase the quark pressure just by the amount needed to counter the effect of the interaction in eq (4.42). Hence we indeed have $\mathrm{T} \sim \mathrm{B}^{1 / 4}$, including the strange quarks (see the discussion below eq $(4.46))$. tends to

Let us here further note that for $T \ll \mu$ the baryon chemical potential

$$
\mu=3 \mu_{q} \Rightarrow 3 B^{1 / 4}\left[\frac{2 \pi^{2}}{\left(1-\frac{2 \alpha_{S}}{\pi}\right)}\right]^{1 / 4}=1320 \mathrm{MeV}\left[\alpha_{S}=1 / 2, B^{1 / 4}=190 \mathrm{MeV}\right] .
$$

In concluding this discussion of the $P=0$ line of the quark-gluon plasma, let us note that the choice $\alpha_{s} \sim 1 / 2$ is motivated by the fits to the charmonium and upsilonium spectra as well as to deep inelastic scattering. In both these cases space-like domains of momentum transfer are explored. The much smaller value of $\alpha_{s} \sim 0.2$ is found in time-like regions of momentum transfer in $\mathrm{e}^{+} \mathrm{e}^{-+}$ hadron experiments. We recall that this was the behavior derived from eq (3.17) (see fig. 3.1). In the quark-gluon plasma, described up to first order perturbation theory, positive and negative momentum transfers occur: the perturbative corrections to the radiative $T^{4}$ contribution is dominated by time-like momentum transfers, while the correction to the $\mu^{4}$ term originates from space-like quark-quark scattering. Hence it is preferable that two different values of $\alpha$ be used in the above given expressions.

Consider now the energy density at $\mu=0$. We find the simple result, restating again some factors

$$
\varepsilon(\mu=0)=B+\frac{\pi^{2}}{30} T^{4}\left[{ }_{s}{ }^{8}{ }_{C}\left(1-\frac{15}{4} \frac{\alpha_{S}}{\pi}\right)+2{ }_{I}{ }^{2}{ }_{S}{ }^{3} \frac{7}{4}\left(1-\frac{50}{21} \frac{\alpha_{S}}{\pi}\right)\right] .
$$

We note that in both quarks and gluons the interaction conspires to reduce the effective available number of degrees of freedom. At $\alpha_{s}=0$ we find the handy relation

$$
\varepsilon_{q}+\varepsilon_{g}=\left(\frac{T}{160 \mathrm{MeV}}\right)^{4}\left[\frac{\mathrm{GeV}}{\mathrm{fm}^{3}}\right]
$$

At $\alpha_{S}=1 / 2$ we are seemingly left with only $\sim 50 \%$ of the degrees. of freedom, and the temperature "unit" in the above formula drops to $135 \mathrm{MeV}$. However, as mentioned above, we should rather use $\alpha_{s} \sim .2$ in eq $(4.45)$ in which case the contribution of strange quarks, which is about $30 \%$ of the last term in (4.45) just compensates these interaction effects. Hence (4.46) is the proper rough estimate to be kept in mind. 
So far we have mostly neglected to include heavy flavors into the description. For a charm quark with a mass of about $1500 \mathrm{MeV}$ the thermodynamic abundance is sufficiently low to ignore its influence on the properties of the plasma. While its production is exceedingly slow, even the influence of its equilibrium abundance on the thermodynamic properties of the plasma is quite negligible. Evaluating the phase-space integrals we find that the ratio of charm to light antiflavor (either $\bar{u}$ or $\bar{d}$ ) is

$$
c / \bar{q}=\bar{c} / \bar{q}=e^{-\left(m_{c}-\mu / 3\right) / T}\left(\frac{m}{T}\right)^{3 / 2} \frac{1}{2} \vee \frac{\bar{\pi}}{2} .
$$

Taking as a numerical example $m_{c}=1500 \mathrm{MeV}, T=200 \mathrm{MeV}, \mu=0$ one finds $c / \bar{q}=7.10^{-3}$. Thus the energy fraction carried by the intrinsic plasma charm here would be $\sim 0.2 \%$ and unimportant for the thermodynamic properties of the plasma, but quite significant in direct charm detection experiments. However, the approach to chemical equilibrium (see below) is too slow in nuclear collisions to saturate the phase space even within the most optimistic scenarios, except in circumstances in which $\mathrm{T} \sim \frac{1}{2} \mathrm{~m}_{\mathrm{c}}$ were reached.

Clearly, we must turn our attention to strangness. With a current quark mass of about 150-180 MeV we are actually at the threshold $T=m_{s}$ and indeed one finds that there is a quite appreciable s-abundance. An expicit calculation [4b] has shown that chemical equilibrium will be reached during the short time of a heavy ion reaction. The motion of the particles being already semirelativistic, a significant increase of the number of available degrees of freedom of quarks in eq (4.45) is recorded due to s $\bar{s}$ production. In the case that $T>m_{s}$ we have to increase the number of flavors to 3 while at $T \sim \mathrm{m}_{\mathrm{s}}$ the effective flavor number is 2.8. The appearance of strangenes is a very important qualitative feature and we will return to its discussion in section 7 .

As a final aspect of the perturbative quark-gluon plasma we consider now the role of color charge in the statistical description. We note that for finite-size bags it is essential to ensure the color neutrality of the considered states: much of hadronic structure is a consequence of the requirement of color neutrality and of symmetries of the quark wave functions in the bags. This effect of color interactions we have, however, not yet included into our considerations. The requirement that all physical states accessible to the quark-gluon plasma are color neutral is a very strict requirement. As long as only very few particles are present, color neutral states can be constructed explicitly. But how can we treat an excited, relativistic many-body system? The answer of principle is quite simple: in eq (4.12) the trace has to include only color neutral states. That is, we should consider

$$
z_{C=0}=\operatorname{Tr}_{c=0} e^{-\beta\left(H-\mu_{q} Q\right)} .
$$

However, in order to arrive at a manageable result we had to allow all states in the trace. In order to solve this problem [33] we borrow the main technical idea from the work of Redlich and Turko [34], as first applied in reference [35]. Each state of the Hamiltonian $\mathrm{H}$ can be classified within the 
irreducible subspaces according to its transformation properties under the representations of the color SU(3) group. In order to compute the singlet contribution eq (4.48) we first introduce the generating functional

$$
\tilde{z}=\sum_{c} \frac{1}{d_{c}} x_{c}\left(\phi_{j}\right) z_{c}
$$

where the sum is carried out over all irreducible representations characterized by the index $c$. The variables $\phi_{j}$ of the coefficient functions, i.e., group characters $x_{c}$ will permit the inversion of eq (4.49) through a reTation of the type found when solving for a set of complete orthogonal functions, i.e.

$$
\begin{gathered}
\int d^{n} \phi M\left(\phi_{j}\right) x_{c^{\prime}}^{*}\left(\phi_{j}\right) x_{C}\left(\phi_{j}\right)=\delta_{C C^{\prime}} \\
\sum_{c} x_{c}^{*}\left(\phi_{j}^{\prime}\right) x_{c}\left(\phi_{j}\right)=\delta^{n}\left(\phi^{\prime}-\phi\right)
\end{gathered}
$$

Hence $d_{c}$ in eq (4.49) is a suitable normalization constant (dimension of the representation), while $M\left(x_{i}\right)$ is a function defining the Haar measure. With these relations we have

$$
z_{c}=d_{c} \int d^{n} \phi M\left(\phi_{j}\right) x_{c}\left(\phi_{j}\right) \tilde{z}\left(\beta, V \ldots ; \phi_{j}\right) .
$$

The problem is to obtain a suitable set of functions $\phi_{C}\left(x_{j}\right)$ such that

1) $Z$ eq (4.49) can be explicitly computed, e.g., in the particle number representation, and

2) eq $(4.50)$ is satisfied.

A hint how it is possible to proceed is contained in eq (4.12), since the baryon number operator commutes with the Hamiltonian we could use $\hat{Q}$ in the exponent in order to divide the Hilbert space into sectors of a given baryon number. We proceed now in this fashion with the non-abelian group SU(3). There are two mutually commuting charges, in the standard representation of SU(3) these are the 3 and 8 directions of the color space. We therefore consider the following Ansatz for the generating function

$$
q=\operatorname{Tr}\left(e^{-\beta\left(\hat{H}-\mu_{q} \hat{Q}\right)} \tilde{u}\left(\phi_{3}, \phi_{8}\right)\right)
$$

here

$$
\tilde{u}\left(\phi_{3}, \phi_{8}\right)=e^{-i \phi_{3} \hat{Q}_{3}-i \phi_{8} \hat{Q}_{8}}
$$


where we have introduced the new factor $\tilde{u}$. Since the norm of $\tilde{u}$ is bounded by unity we have no trouble to establish the existance of the generation function (4.52): its absolute value must always be smaller than $z$, obtained replacing $\tilde{u}$ by unity.

It is our first aim now to show that eq (4.52) obtains the desired form (4.49). The Hilbert space spanned by $H$ is a direct product of orthogonal subspaces characterized by the color $c$ of states belonging to each sector (i.e., transformation properties under the $S U(3)_{c}$ group). Hence we can write:

$$
\tilde{Z}=\sum_{c} \operatorname{Tr}_{c}\left[e^{-\beta\left(H-\mu_{q} Q\right)} e^{-i \phi_{3} Q_{3}-i \phi_{8} Q_{8}}\right]
$$

where the sum $c$ is a symbolic sum over all irreducible representations of SU(3), usually characterized by two positive integers $(p, q) \in(0, \infty)$. We can now constrain our discussion to the "good color" subsectors of the entire Hilbert space of the color space. Within each sector, a complete orthonormal set of states is generated in the particle number representation of $H$ :

$$
1_{c}=\sum_{\xi_{c}=1}^{d} \sum_{\nu_{c}}\left|\nu_{c}, \xi_{c}\right\rangle\left\langle\xi_{c}, \nu_{c}\right|
$$

where $d_{c}$ is the degeneracy, i.e., dimensionality of each color multiplet, while $\xi_{c}$ counts all quantum numbers within a given irreducible representation which are related to the internal symmetry. $v_{c}$ is a short-hand notation for the set of states determined by the occupantion numbers $n_{j}$ or $i^{\text {th }}$ momentum state. Inserting eq (4.54) into (4.53) we find

$$
\begin{aligned}
\tilde{z}=\sum_{c}\left\{\sum_{\xi_{c}} \sum_{\nu_{c}} \sum_{\xi_{c}^{\prime}} \sum_{c}\left\langle\xi_{c}, \nu_{c}, e^{-\beta\left(H-\mu_{q} Q\right)} \mid \nu_{c}^{\prime}, \xi_{c}^{\prime}\right\rangle\right. \\
\\
\left.\quad x\left\langle\xi_{c}^{\prime}, \nu_{c}^{\prime}\left|e^{-i \beta \phi_{3} Q_{3}-i \beta \phi_{8} Q_{8}}\right| \nu_{c}, \xi_{c}\right\rangle\right\} .
\end{aligned}
$$

The first factor in eq (4.55) cannot depend on $\xi_{c}$ as we had explicitly assumed exact color symmetry; hence it is diogonal in $\xi$. Similarly, the second factor

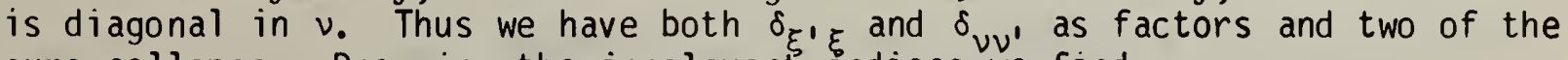
sums collapse. Dropping the irrelevant indices we find

$\tilde{z}=\sum_{c}\left\{\sum_{\nu_{c}}\left\langle\nu_{c}\left|e^{-\beta\left(H-\mu_{q} Q\right)}\right| \nu_{c}\right\rangle \sum_{\xi_{c}=1}^{d_{c}}\left\langle\xi_{c}\left|e^{-i \phi_{3} Q_{3}-i \phi_{8} Q_{8}}\right| \xi_{c}\right\rangle\right\}$. 
Recalling that by definition

$$
z_{c}=\operatorname{Tr}_{c} e^{-\beta\left(H-\mu_{q} Q\right)}=d_{c} \sum_{\nu_{c}}\left\langle\nu_{c}\left|e^{-\beta\left(H-\mu_{q} Q\right)}\right| \nu_{c}\right\rangle
$$

we find the desired decomposition (4.49) with

$$
x_{C}\left(\phi_{3}, \phi_{8}\right)=\sum_{\xi_{C}=1}^{d_{C}}\left\langle\xi_{C}\left|e^{-i \phi_{3} Q_{3}-i \phi_{8} Q_{8}}\right| \xi_{C}\right\rangle
$$

being now recognized as the character of the irreducible representation. The relations (4.50) are automatically satisfied for all compact semi-simple Lie groups. Even without the use of methods of group theory one can easily verify in particular cases that eq (4.58) indeed defines a suitable set of functions. Regressing for a short moment to the SU(2) Lie group we easily find

$$
x_{j} \operatorname{SU}(2)\left(\phi_{3}\right)=\sum_{m_{z}=-J}^{+J} e^{-i \phi_{3} m} z=\frac{\sin (2 J+1) \phi_{3}}{\sin 1 / 2 \phi_{3}}
$$

Hence, with the Haar measure [36]

$$
M^{S U(2)}\left(\phi_{3}\right)=\frac{1}{\pi} \sin ^{2} \phi_{3} / 2, \phi_{3} \in(0,2 \pi)
$$

equations (4.50) are easily verified. At this point the surprise is that alone the eigenvalues of $e^{-i \phi_{3} I_{3}}$ fix the irreducible representation of $\mathrm{SU}(2)$. However, we note that the rank of the representation enters explicitiy in eq (4.59) and, of course, into the expression (4.58) for SU(3).

The crucial point of this approach is the fact that the generating function $Z$, eq (4.53) can be explicitly determined! Actually the steps are identical to those used above, c.f., eqs (4.16)-(4.21). The reader is invited to repeat the derivation now with complex quantities i $\phi$ instead of the chemical potential. One simply finds for quarks the analogue of eq (4.21):

$$
\ln \tilde{z}_{q}=\sum_{f 1 \text { avor }} 2_{s} V \int \frac{d^{3} p}{(2 \pi)^{3}} \sum_{c, b} \ln \left[1+\exp \left(-\beta\left(\sqrt{p^{2}+m_{f}^{2}}-\mu b\right)-i \alpha_{c}\right)\right]
$$


for colored quarks. Instead of a color factor 3 . we now find the suin over th: raigenvalues $\alpha_{j}$ of the charges $Q_{3}, \eta_{8}$; in the triplet $(c \in(1,0))$ and ant $i$ triplet $(c \in(0,1))$ representation. In the triplet representation for which $b=+1 / 3$ we have

$$
\begin{aligned}
& \alpha_{R}=\phi_{1} / 2+\phi_{2} / 3 \\
& \alpha_{G}=-\phi_{1} / 2+\phi_{2} / 3 \\
& \alpha_{B}=-2 / 3 \phi_{2}
\end{aligned}
$$

where $R, B, G$, refers tis the usual red, blue, green colors. In the antitriplet representation in which $b=-1 / 3$ the sign of all the three angles reverses. We note that except when $\mu=0$, the generating function $Z$, eq $(4.61)$ will not be real. However, the integration over the group with the proper Haar measure leads to a real resuit for the partition function $Z$. This measure is in the case given here

$$
\begin{aligned}
d^{2} \phi M\left(\phi_{1}, \phi_{2}\right)= & \frac{8}{3 \pi^{2}} d\left(\frac{\phi_{1}}{2}\right) d\left(\frac{\phi_{2}}{3}\right)\left[\sin \frac{1}{2}\left(\frac{\phi_{1}}{2}+\phi_{2}\right) \sin \frac{\phi_{1}}{2} \sin \frac{1}{2}\left(-\frac{\phi_{1}}{2}+\phi_{2}\right)\right]^{2} ; \\
& \phi_{1}, \phi_{2} \in[-\pi, \pi]
\end{aligned}
$$

We note that for massless quarks, i.e., neglecting the strange quark fraction eq (4.61) can be evaluated analyticaTiy following the results of eq (4.2.2) and replacing $\mu \rightarrow \mu \pm i \alpha$. In order to judge the influence of the color conservation it is sufficient to consider computationlally the particular case of a baryon-less, $\mu=0$, plasina droplet. In this case we find, substituting $\mu / T \rightarrow i \alpha$ in eq (4.2?)

$$
\begin{aligned}
\ln \tilde{z}_{q} & =\ln \tilde{z}_{q}^{(0)} \ln \tilde{Z}_{q}^{(1)} \\
\ln \tilde{z}_{q}^{(0)} & =V T^{3} \pi^{2} \frac{7}{30} \\
\ln \tilde{Z}_{q}^{\left({ }^{1}\right)} & =V T^{3} \frac{\pi^{2}}{3} \sum_{i=R, G, B}\left[\frac{1}{2}\left(\frac{\alpha_{i}}{\pi}\right)^{4}-\left(\frac{\alpha_{i}}{\pi}\right)^{2}\right]
\end{aligned}
$$


Here $\ln \tilde{z}_{\mathrm{g}}^{0}$ is the partition function without the color constraint while $\ln Z_{q}^{(1)}$ vanishes at $\alpha_{j}=0$.

It is important to appreciate that in the quark-gluon plasma the projection, eq (4.51) on a good color sector has to be carried out for both quarks and gluons simultaneously. Hence one has to carry out the integral

$$
z_{c}=\frac{1}{d_{c}} \int d^{2} \phi M\left(\phi_{1}, \phi_{2}\right) x_{c}(\phi) \tilde{z}_{q}(\beta, V, \mu ; \phi) \tilde{z}_{g}(\beta, V ; \phi)
$$

where for the singlet, $c=0$, the character $x_{0}$ is just unity. In order to obtain $Z_{g}$ we have to evaluate the analogue of eq (4.29)

$$
\ln \tilde{Z}_{g}=2 \sum_{i=1}^{8} V \int \frac{d^{3} p}{(2 \pi)^{3}} \ln \left(1-e^{-\beta|p|-i \alpha_{i}}\right)
$$

where the color sum $i$ runs over the octet of gluons with the eigenvalues of the $Q_{3}, Q_{8}$ charges in the octet representation $(1,1)$.

As the octet results from the product of the triplet and anti-triplet representations of $\mathrm{SU}(3)$, omitting the singlet, we have simply

$$
\alpha_{i}^{g}=\alpha_{j}-\alpha_{k}, j, k \in(R, B, G)
$$

taking the six non-zero and two zero angles $\alpha_{i}^{g}$. Explicitly

$$
\begin{aligned}
\alpha_{i}^{g} & =\left(\alpha_{R}-\alpha_{G}, \alpha_{G}-\alpha_{B}, \alpha_{B}-\alpha_{R},\right. \\
& \left.-\left(\alpha_{R}-\alpha_{G}\right),-\left(\alpha_{G}-\alpha_{B}\right),-\left(\alpha_{B}-\alpha_{R}\right), 0,0\right) .
\end{aligned}
$$

We therefore find

$$
\begin{aligned}
& \ln \tilde{Z}_{g}=\ln \tilde{z}_{g}^{(0)}+\ln \tilde{Z}_{g}^{(1)} \\
& \ln \tilde{Z}_{g}^{(0)}=V T^{3} \pi^{2} \frac{8}{45} \\
& \ln \tilde{z}_{g}^{(1)}=V T^{3} \frac{\pi^{2}}{6} \sum_{i=1}^{3}\left[-\frac{1}{2}+\left(\frac{\left|\alpha_{j}^{g}\right|}{\pi}-1\right)^{2}-\frac{1}{2}\left(-\frac{\left|\alpha_{j}^{g}\right|}{\pi}-1\right)^{4}\right]
\end{aligned}
$$


We note that the color sum in eq (4.68) was simplified and includes now only the first three terms of eq (4.67b). Again, $z_{g}^{(0)}$ is the partition function without the color constraint eq $(4.30)$, while $\ln z_{q}^{(1)}$ vanishes at $\alpha_{q}^{g}=0$.

From the preceeding derivations the enormous influence of color neutrality is apparent. Practically all states have to be rejected when evaluating the trace (4.48) constrained to color singlets. A global quarkgluon color correlation is introduced in view of eq $(4.65)$. Both quark and gluon fractions of the plasma separately can have color as long as overall neutrality is assured. In the limit $V \rightarrow \infty$ one can advance arguments that invalidate this conclusion concerning the importance of color neutrality. To wit, color fluctuations can not be large; hence, the influence of the requirement of exact color conservation should not be felt. While this argument can be proven easily analytically it turns out [33] that the limit $V \rightarrow \infty$ actually means

$$
V T^{3}>10
$$

To illustrate this point we show in figure 4.1 the energy density as derived from eq (4.65) and divided by $\sigma T^{4}$

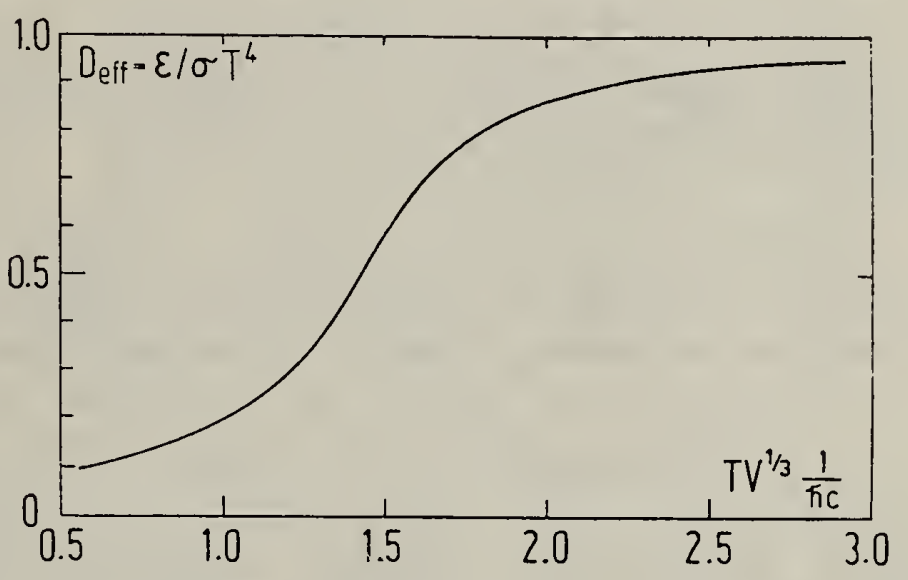

Fig. 4.1 Relative degeneracy $D_{\text {eff }}$ of the quarkgluon plasma, as a function of $\mathrm{TV}^{1 / 3}$.

$$
\sigma=\left(2 \times 8+2 \times 2 \times 2 \times 3 \times \frac{7}{8}\right) \pi^{2} / 30
$$

for quark-gluon plasma, C.f. eq (4.45). We observe that at TV $1 / 3 \sim 1.5$ only half of the expected number of excitations is available. That means that since we anticipate $T \sim 150-170 \mathrm{MeV}$, one has to deal with the finite voiume problems and color constraints whenever clumps of hadronic matter are produced with baryon number $b \lesssim 10$. In particular this concerns the study of the transition from the plasma state to the hadronic gas; color configurations and correlations here will be of great practical relevance. Work along these lines is in progress. Therefore the discussion of different hardonic phases given below by necessity can be only qualitative in nature. But before we turn to this issue we will consider the kinematic conditions for the formation of the plasma in nuclear collisions. 


\section{QUARK-GLUON PLASMA FORMATION IN NUCLEUS-NUCLEUS COLLISIONS}

From the limited information coming from cosmic ray data [37], and from our knowledge of the nucleon-nucleus interaction [38] we take as the starting point the observation that the normal hadronic cross sections are not large enough, i.e., the mean free path in hadronic matter is too large, to confine a high-density region by multiple scattering. We record, however, here the recently observed rather narrow rapidity distributions at $\sqrt{s}=540 \mathrm{GeV}$ from the CERN pp collider [39] which indicate non-transparency at a level not anticipated before. In order to create a larger size high-density region a quark gluon plasma seed [12] must have been formed by a statistical fluctuation. Thereafter the plasma can begin to grow by capture of the trailing nucleons of the colliding nuclei. In such a scenario, the densest plasma will result when the seed is formed early in the collision, i.e., in the central rapidity region for symmetric collisions $\left(A_{p}=A_{t}\right)$. - However, plasma production will occur according to this mechanism with a non-negligible distribution towards projectile and target rapidity limits. In events with early plasma seed the baryon number content of the plasma would be appreciable for large nuclei, peaking in the central rapidity region. A different scenario would arise in events in which the plasma state is not ignited; here the baryons would be found mostly in projectile-target regions of the rapidity, owing to the expected substantial transparancy of normal nuclei to high energy particles [38]. Still, the high radiation energy density reached in such collisions could also lead to a baryonless plasma, as discussed by others [11].

In order to fulfill its role the above introduced seed must indeed be a high particle density region similar to the quark-gluon plasma, albeit small in size, with sufficiently thermalized momentum distributions and with some color deconfinement; however, chemical equilibrium between different particle species, i.e., quark flavors, is not required. In such a case the quark mean free path, $\bar{\lambda}$, can become comparable to the seed size, $R$, and we can have $R / \lambda \gtrsim 1$. Occasional formation of such seeds is assured by inspection of actual numerical results obtained with relativistic cascade calculations [40]. We have good reason to believe not only in occasional, but perhaps even in relatively frequent, creation of such a seed, through an accidental local large fluctuation of particle density over the hadronic volume.

The energy influx to the plasma seed is controlled by the nuclear matter inflow. We consider here a) the kinematic conditions for the occurrence of the instability, seed $\rightarrow$ plasma, and b) the maximum achievable temperature in the most favorable case. For this purpose we do not need to consider the influence of the likely increase of the energy and particle density of the projectile or target in their rest frames arising from the entrance channel interactions. In order to err on the conservative side we compute as if all of the interacting region would instantly turn into the plasma state without compressions of nuclear degrees of freedom. Namely, if the formation of the seed is delayed, the increase of the densities would make the environment even more suitable for the occurrence of the plasma seed. However, the crucial condition to be respected follows from the observation that once the seed is there it can lead to a large-scale plasma state only if the energy loss of the seed is exceeded by its energy gain. Even below this "sharp" boundary defined as the instability without nuclear compression in target or projectile, 
occasional formation of plasma drops in the dense regions of compressed nuclear matter will occur. These precursor phenomena will smear out the kinematic limit, otherwise already spread out by fluctuations of the seed location, range of the impact parameters, etc. We believe that a detailed discussion of these effects is premature. Therefore we now determine the conditions which must be fulfilled for the ignition of a large-scale central plasma state.

While the plasma receives energy and baryon number by the nucleons impacting on it, it also inevitably loses energy by thermal radiation. Thus, in order to grow there must hold for the total plasma energy $E$,

$$
\frac{d E}{d t}=\frac{d E^{A}}{d t}-\frac{d E^{R}}{d t}>0
$$

where $d E^{A} / d t$ is the heating by the incoming nucleons absorbed in the seed, and $\mathrm{dE}^{\mathrm{R}} / \mathrm{dt}$ is the energy loss by thermal radiation. If $\mathrm{dE} / \mathrm{dt}$ is negative the plasma will fizzle rather than grow. We now discuss the two terms, beginning with the gain term.

The energy influx into the plasma is controlled by the nuclear fourvelocity $u^{\nu}=\gamma(1, \vec{v})$, the plasma surface normal vector as seen from the CMframe, $n^{\mu}=(0, \hat{n})$ the nuclear energy-momentum tensor $T_{\mu \nu}$ and the probability for the absorption of an incoming nucleon by the piasma, a. Thus we have, with $d^{2} A$ the surface element,

$$
\frac{d E^{A}}{d t}=\int d^{2} A\left(-T_{\mu \nu} u^{\mu} n^{\nu} a\right)
$$

As is well known

$$
T_{\mu \nu}=\varepsilon_{0} u_{\mu} u_{\nu}+p\left(u_{\mu} u_{\nu}-g_{\mu \nu}\right)
$$

where $\varepsilon_{0}$ and $p$ are the energy density and the pressure ( $p$ included here for completness only) in the rest frame of the projectile or target nucleus, respectively. Hence we have

$$
T_{\mu \nu} u^{\mu} n^{\nu}=\rho_{0} m \gamma \vec{n} \cdot \vec{v}
$$

where $\rho_{0}$ is the equilibrium nuclear density, i.e., $\rho_{0}=1 / 6 \mathrm{fm}^{-3}$. Furthermore, seen from the CM frame and expressed in Eerms of the projectile laboratory energy per nucleon, $E_{p}$, we have 


$$
\begin{gathered}
v=\left(\frac{E_{p}-m}{E_{p}+m}\right)^{1 / 2} \\
\gamma=\frac{\left(2 E_{p} m+2 m^{2}\right)^{1 / 2}}{2 m}
\end{gathered}
$$

The absorption coefficient a is assumed, as usual, to be

$$
a(z)=1-e^{-z / \lambda}
$$

where $z$ is the thickness of the plasma region and $\lambda$ is the absorption length of a hadron in the plasma. When weighted with $\vec{n} \cdot \vec{v}$ over the plasma surface this leads to

$$
\bar{a}(R)=\frac{1}{2}\left\{1+2 e^{-2 R / \lambda}\left[\frac{\lambda}{2 R}+\left(\frac{\lambda}{2 R}\right)^{2}\right]-2\left(\frac{\lambda}{2 R}\right)^{2}\right\} .
$$

The overall factor $1 / 2$ above reflects the ratio between the surface of a circle with radius $R$ and a half sphere, for $\lambda / R \rightarrow 0$. The absorption coefficient $a(R)$ is indeed the average absorption probability. Through $\lambda$ it depends on the particle density in the plasma, i.e., temperature and baryon density. Still, the gain term in eq (5.1) depends mainly on the projectile energy. The final expression is, in detail,

$$
\begin{aligned}
\frac{d^{3} E^{A}}{d^{2} A d t} & =\frac{1}{2} \rho_{0}\left(\frac{E_{p}-m}{E_{p}+m}\right)^{1 / 2}\left(2 E_{p} m+2 m^{2}\right)^{1 / 2} \\
& \times \frac{1}{2}\left[1+2 e^{-2 R / \lambda}\left(\frac{\lambda}{2 R}+\left(\frac{\lambda}{2 R}\right)^{2}\right)-2\left(\frac{\lambda}{2 R}\right)^{2}\right] .
\end{aligned}
$$

We now turn to the description of the energy loss term of eq (5.1). In general, two mechanisms for energy loss from a plasma are possible, viz., adiabatic expansion and thermal radiation. At least in the beginning, i.e.e, at the time of decision between ignite and fizzle, the expansion should pTay no role as the impacting nucleons provide an inertial confinement for the plasma. However, pion evaporation from the plasma is still possible, and the cooling associated with this process provides the energy loss of eq (5.1). Of course, some of the emitted pions will be returned to the plasma by the 
incoming nucleons. However, this return will be too late to have an impact on the question fizzle or grow: once the process has fizzled, i.e., the plasma seed has hadronized, the collision is back to the hadron cascade regime. On the other hand, if plasma growth has taken place the returning pions will of course return their evaporation energy to the plasma and contribute to the ultimate energy density of the plasma. Also, the influence of expansion has to be reconsidered then.

We now describe the microscopic mechanism of the pion evaporation process and estimate the magnitude of the energy loss [14]. To wit: when a fast quark or antiquark hits the boundary it can form a jet-like structure filled with color field flux, i.e., a fluxtube. For sufficiently high quark momentum this tube instead of reträcting tears apart by qव pair creation. The leading particle associates with the antiparticle of the pair to form a meson, while the remaining pair particle may retract into the plasma. By this process in effect a fast quark of the plasma is replaced by a slow quark, the total number of quarks in the plasma having remained constant. If the so emitted particle is a pion, rather than a "heavy" meson, the probability for the flux tube to break is greatly influenced by the gain in binding of the final $q \bar{q}$ state. In this case the necessary momentum is determined by kinematic considerations, i.e., the produced pion must be able to take away not only the energy but aiso the appropriate momentum.

We now develop a quantitative model suitable for surface temperatures of 150 - $220 \mathrm{MeV}$ and moderate baryon densities, such that the particle density is less than $\sim 10$ particles $/ \mathrm{fm}^{3}$. Under these circumstances surface collisions involving more than one particle per $\mathrm{fm}^{2}$ are rare. Hence He can limit ourselves to consider sequential one-particle events. In order for the surface collision to lead to pion emission the particle momentum normal to the surface must exceed a certain threshold. In particular, this momemtum has to be larger than the normal momentum of the emitted pion. We take this threshold momentum to be of the order of $1 / 4 \mathrm{GeV} / \mathrm{c}$ for quarks leading to pions; our results are quite insensitive to the precise choice, as well as to the actual shape of the threshold function $\theta$ describing the probability of pion emission. Hence we will use:

$$
\theta(p)=\left\{\begin{array}{ll}
1, & p_{\perp}>p_{M} \sim 1 / 4 \mathrm{GeV} \\
0, & 0<p_{\perp} \lesssim p_{M}
\end{array} .\right.
$$

We note that the average energy (momentum) of practically massless quarks is about 3T 500-650 MeV and that the particle densities peak at $\sim 2 \mathrm{~T}$. Hence almost half of all quarks and antiquarks can participate in the radiation cooling.

The energy per unit surface and unit time that leaves the quark-gluon plasma is now simply given by

$$
\frac{d^{3} E}{d^{2} A d t}=g \int \frac{d^{3} p}{(2 \pi)^{3}} \rho(p) f(E) E(p) \theta(p) \frac{d^{3} V}{d^{2} A d t}
$$


where $g$ is the degeneracy. As only light quarks lead to the dominant pion channel we have $g=3 c^{\times 2} s^{\times 2} f=12 . \quad p(p)$ is the phase space quark and antiquark particle density

$$
p(p)=\left[\exp \left(\left(p-\mu_{q}\right) / T\right)+1\right]^{-1}+\left[\exp \left(\left(p+\mu_{q}\right) / T\right)+1\right]^{-1}
$$

The differential is simply the normal velocity of particles impinging on the plasma surface

$$
\frac{d^{3} V}{d^{2} A d t}=\frac{d^{2} A d z}{d^{2} A d t}=\frac{d z}{d t}=v_{\perp}=\frac{p_{\perp}}{E(p)}=\frac{p_{\perp}}{\left(p_{\perp}{ }^{2}+p_{\|}{ }^{2}\right)^{1 / 2}}
$$

Since the energy leaving the plasma region is not the total energy contained in the leading particle we have in (5.11) included the efficiency factor $f$. In the present case only one pair is created to form the emitted pion. A naive degree-of-freedom counting leads to $f * 2 / 3$. f probably approaches unity for very high energy leading particles. We disregard the energy dependence of $f$; choosing the value $\bar{f}=2 / 3$ we obtain a lower limit on the energy transfer.

In view of the uncertainties it is sufficient to expand in eq (5.12) the Fermi distributions and to retain only the Boltzmann term for the $q$, $q$ distribution:

$$
\rho(p) \cdot 2 \cosh (\mu / T) e^{-\sqrt{p_{\|}^{2}+p_{1}^{2}} / T} .
$$

We find that this overestimates the final result by about $5-10 \%$. Combining eqs (5.10) and (5.13) with eq (5.14) we obtain the generalized StefanBoltzmann law:

$$
\begin{aligned}
\frac{d^{3} E}{d^{2} A d t} & =\bar{f} g 2 \cosh \left(\mu_{q} / T\right) \int_{P_{M}}^{\infty} \frac{d p_{\perp}}{(2 \pi)} p_{\perp} \int_{0}^{\infty} \frac{p_{\|} d p_{\|}}{(2 \pi)} e^{-\sqrt{p_{\|}{ }^{2} p_{\perp} 2 / T}} \\
& =\bar{f} \frac{g}{2 \pi^{2}} \cosh (\mu / T) T^{4} 3 e^{-p_{M} / T}\left(\frac{1}{3}\left(\frac{p_{M}}{T}\right)^{2}+\left(-\frac{p_{M}}{T}\right)+1\right) .
\end{aligned}
$$


In figure 5.1 we show the cooling rate calculated from eq (5.15) as a function of the surface temperature $T$, choosing $\mu_{q} / T=1$. For $\mu_{q} \approx 0$ the values arelower by about $30 \%$ : in a baryonless plasma at a given temperature the reduced $q$-density is not entirely compensated by the enhanced $\bar{q}$ density. We see that indeed the precise value of $P_{M}$, or, said differently, the precise form of the threshold function $\theta$, eq (5.10), does not matter. Concerning the choice of $\mu_{\mathrm{q}}$ we note that even though equilibrium baryon density does not very likely prevail, the best choice for $\mu_{\mathrm{g}}$ in the initial stages of the plasma formation would be $\mu_{\mathrm{g}} \lesssim M_{N} / 3 \approx 2 T$, consistent with the non-degeneracy assumption for $T \sim \frac{1}{6} M_{N}$. As local thermalization occurs, $\mu_{q}$ diminishes and approaches $\mathrm{T}$.

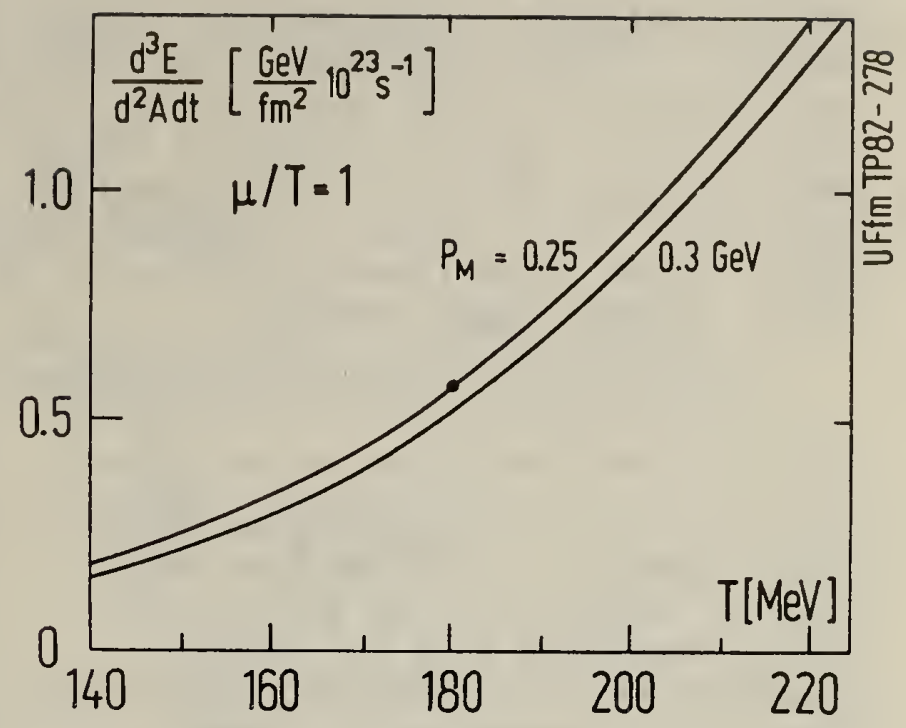

Fig. 5.1 Pion radiation surface brightness as function of temperature.

Before returning to the ignition condition given by the inequality (5.1) we discuss our result in terms of a numerical example chosen to represent a typical case of a quark-gluon plasma. Our example is a spherical plasma droplet of $R=4 \mathrm{fm}$, a surface temperature of $T=180 \mathrm{MeV}$, and $\mu / T=1$. The energy density then is $2.1 \mathrm{GeV} / \mathrm{fm}^{3}$ according to eq (4.34) and recalling that strange quarks compensate for a large part of the interaction which is of order $0\left(\alpha_{s}\right)$. The baryon density is according to eq $(4.34), \sim .5 / \mathrm{fm}^{3}$, i.e.., about $3 \rho$. The baryon number exceeds 150 if $T$ is larger in the interior. Since $0.7 \mathrm{GeV} / \mathrm{fm}^{3}$ is needed for creation of the final baryons implied by the assumed value of $\mu$, the available energy density is about $1.4 \mathrm{GeV} / \mathrm{fm}^{3}$ and the total available energy is ca. $400 \mathrm{GeV}$. For this example we find for the rate of energy loss through the surface

$$
\frac{d E}{d t}=A \bar{f} 0.29 \frac{\mathrm{GeV}}{\mathrm{fm}^{2}} \frac{\mathrm{c}}{\mathrm{fm}}=A 0.58 \frac{\mathrm{GeV}}{\mathrm{fm}^{2}} 10^{+23} \mathrm{sec}^{-1}
$$

We note that this confirms the assumption of a sequential individual-particle process: when one particle of $0.29 \mathrm{GeV}$ impinges on a surface area of $1 \mathrm{fm}^{2}$ the next particle following it with light velocity would be behind by a distance of one fm (i.e., several mean free paths). On the other hand, this indeed is a very large energy loss rate. In our example, the energy loss in the first $10^{-23} \mathrm{sec}$ is $\left(A=200 \mathrm{fm}^{2}\right)$

$$
\Delta t \frac{d E}{d t}=120 \mathrm{GeV} \text {, }
$$


which represents a substantial fraction of the total available energy of about $400 \mathrm{GeV}$. Clearly the smaller the plasma droplet, the more relevant becomes the radiation loss for the lifetime of the plasma. As the available excitation energy scales with $R^{3}$ and the radiation loss with $R$, a small plasma droplet of $b \sim 18, R \sim 2 \mathrm{fm}$ and available energy $40 \mathrm{GeV}$ radiates $30 \mathrm{GeV}$ in the first $10^{-23} \mathrm{sec}$. Hence we are led to urge that experiments involving very heavy nuclei be performed to allow for the creation of sufficiently longlived (i.e., large) plasma regions.

We now return to the discussion of the ignition conditions: we set $\frac{d E}{d t}=0$ in eq (5.1). In figure 5.2 we show the minimum size a plasma seed must have in order for it to grow, i.e.., the minimum seed size for plasma ignition, as a function of projectile energy for a selection of plasma ignition temperatures, $T_{I}$, computed taking $\mu / T=2$. In the initial stages of the nuclear collision this is the more likely nature of the parameters. $q$ dominate $q$ and we err on the conservative side by enhancing the radiation losses by that choice. While at density of $2 \mathrm{GeV} / \mathrm{fm}^{3}$ in the plasma the particle density is about $4 / \mathrm{fm}^{3}$ leading to $\lambda \sim \frac{1}{3}-\frac{1}{2} \mathrm{fm}$, we anticipate that in the initial stages of the collision we have a particle density of about $1 / \mathrm{fm}^{3}$ and hence $\lambda \sim 1-1.5 \mathrm{fm}$. For $R / \lambda \sim 1$ we notice that at $T_{I} \sim 150-160 \mathrm{MeV}$ beam energies of $20-50 \mathrm{GeV} /$ nucleon should suffice to lead to plasma ignition

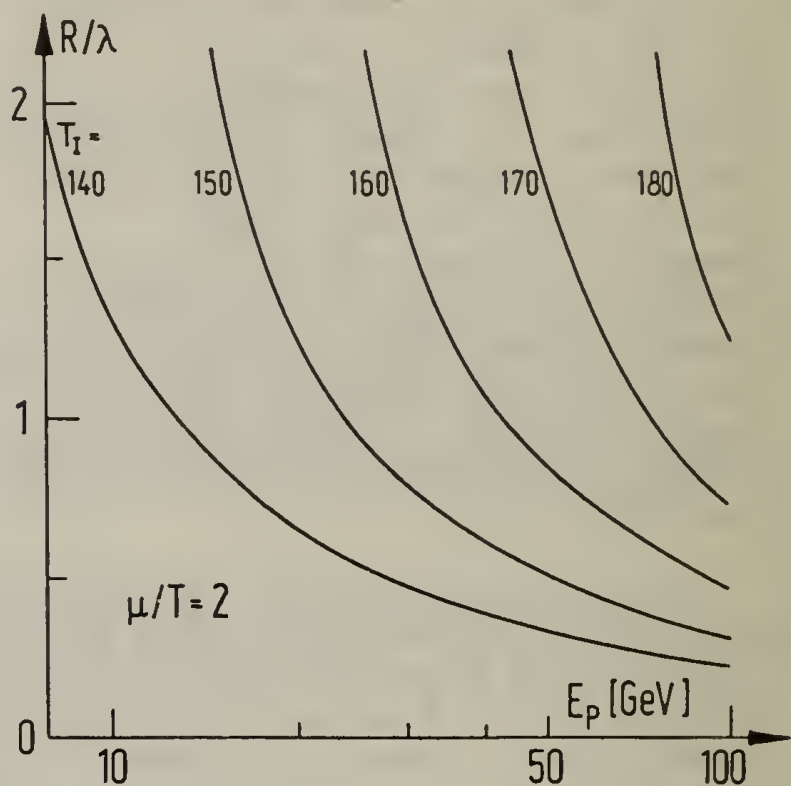

Fig. 5.2 Minimum size of a plasma seed as function of beam laboratory energy for different radiation temper atures. with $R \sim \lambda$. We note that the seed size considered is of the order of the nucleon size. We note that the obtained lower limits for the heavy ion kinetic energy is above the kinematic limit obtained neglecting the loss term in eq (5.1) and requiring an ignition termperature of $160 \mathrm{MeV}$. On the other hand, it seems rather unlikely that ignition $c$ an be achieved at much lower beam energies if the phase transition is of first order. Thus below our limit the collision will fizzle and we just achieve a superheated nucleon gas.

Once the plasma has ignited the temperature of the plasma will grow until the nuclear collision terminates or until the temperature has risen to a level at which the pion radiation overwhelms the energy influx. At this point one must re-examine the question of the cooling mechanism, i.e., first, whether the evaporation of pions will lead to a cooling off of the surface and hence to a shut-off of the evaporation process, or whether the plasma heat conductivity is sufficiently large to maintain a surface temperature high enough for pion radiation to continue; second, whether other processes, principally expansion, contribute substantially to the cooling process. 
We begin by considering the heat conductivity. Since the plasma consists of rather free particles the naive expectation is that a sufficently high conductivity obtains. Indeed, the basic relation between the heat flow $Q$ and the energy density $\varepsilon$ is

$$
\vec{Q}=\ell \vec{\nabla} \varepsilon\left(T ; \mu_{q} / T\right)
$$

where $\ell$ is the mean free path. Assuming that only a radial gradient of $T$ exists, with $\mu_{q} / T \sim$ const over the volume, the radiation equilibrium requires

$$
\frac{d^{3} E}{d^{2} A d t}=Q_{r}=\ell \frac{\partial T}{\partial r} \frac{\partial \varepsilon}{\partial T}=\ell \frac{1}{T} \frac{\partial T}{\partial r} 4 \varepsilon
$$

In our numerical example the required temperature gradient at the surface is, with $\ell$ in the $r$ ange $1 / 2-1 / 3 \mathrm{fm}$ :

$$
\frac{\partial T}{\partial r}=\frac{T}{\ell} \frac{0.215 \mathrm{GeV} / \mathrm{fm}^{3}}{4 \times 2.1 \mathrm{GeV} / \mathrm{fm}^{3}}=(9.2-13.8) \frac{\mathrm{MeV}}{\mathrm{fm}} .
$$

It appears that this temperature gradient is just within sensible bounds, leading for a plasma radius of $4 \mathrm{fm}$ to a temperature differential between the origin and the surface of $\sim 20-30 \mathrm{MeV}$. We further note that unlike in nonrelativistic gases, the mean free path $\&$ here is inversely proportional to $\partial \varepsilon / \partial T$ since it is inversely proportional to the particle density. For $\mu_{\mathrm{g}} / \mathrm{T}<2$ the energy per particle in the plasma is just $3 T$ and hence the particle density $p=\varepsilon / 3 T$. Therefore the necessary temperature gradient, eq (5.17), turns out to be

$$
\frac{\partial T}{\partial r}=\frac{d^{3} E}{d^{2} A d t} \frac{1}{12} \bar{\sigma}
$$

where $\bar{\sigma}$ is the average particle-particle cross section. The range of values given above for $\frac{\partial T}{\partial r}$ corresponds to $\bar{\sigma} \sim \frac{1}{2}$ to $\frac{2}{3} \mathrm{fm}^{2}$.

We now turn to the discussion of the adiabatic expansion of the plasma. To begin with one must recognize that in contrast to the above discussed pion radiation process the expansion requires a collective flow, i.e., a flow in which a hydrodynamic velocity is superimposed over the random thermal motion of all the quarks and gluons. Therefore the relevant time constant is given by the speed of sound and thus is about three times larger than the radiation time constant. Furthermore, the expansion is driven by the excess of the internal pressure over that exerted on the surface by the physical vacuum. Now, the effect of the internal pressure on the surface is reduced by the pion 
radiation. The point is that those particles which penetrate the surface do not exert their full force on the surface. We now demonstrate that they are responsible for a substantial fraction of the internal surface pressure.

Balancing the momenta at the surface we find that instead of $2 p_{\perp}$ the momentum transferred to the surface is

$$
\Delta p=\left\{\begin{aligned}
2 p_{\perp} & : p_{\perp}<p_{M} \\
2 p_{\perp}(1-f): & p_{\perp}>p_{M}
\end{aligned}\right.
$$

where $f$ is the fraction of the normal momentum carried away by the emitted pion. We now recompute the effective pressure on the plasma surface:

$$
\begin{aligned}
\bar{p}_{q} & =g\left[\int_{0}^{p_{M}} \frac{d p_{\perp}}{(2 \pi)} 2 p_{\perp} v_{\perp} \frac{p_{\|} d p_{\|}}{(2 \pi)^{2}} \rho(p)\right. \\
& \left.+(1-f) \int_{p_{M}}^{\infty} \frac{d p_{\perp}}{(2 \pi)} 2 p_{\perp} v_{\perp} \int_{0}^{\infty} \frac{p_{\|} d p_{\|}}{(2 \pi)^{2}} \rho(p)\right]
\end{aligned}
$$

where we have used eq $(5.13)$. We notice that the effective pressure $\bar{p}$ is equal to the expected quark pressure $P_{q}=1 / 3 \varepsilon_{g}$, reduced by the contribution of high normal momentum particles, weighted by the factor $f$ :

$$
\bar{p}_{q}=p_{q}-f g \int_{p_{M}}^{\infty} \frac{d p_{\perp}}{(2 \pi)} 2 p_{\perp} v_{\perp} \int_{0}^{\infty} \frac{p_{\|} d p_{\|}}{(2 \pi)^{2}} \rho(p) .
$$

The important point to realize is that the contributions of particles with $P_{1}>P_{M}$ to the particle pressure $P_{q}$ are dominant. To see this we evaluate, in obvious notation,

$$
\begin{aligned}
& \frac{p_{q}\left(p_{\perp}>p_{M}\right)}{p_{q}} \equiv \frac{\int_{p_{M}}^{\infty} d p_{\perp} p_{\perp}{ }^{2} \int_{0}^{\infty} \frac{p_{\|} d p_{\|}}{\sqrt{p_{\perp}{ }_{1} p_{\perp}}} \rho(p)}{\int_{0}^{\infty} d p_{\perp} p_{\perp}{ }^{2} \int_{0}^{\infty} \frac{p_{\|} p_{\|}}{\sqrt{p_{\perp}{ }^{2} p_{\|}^{2}}} p(p)}=\frac{\int_{p_{M}}^{\infty} d p_{\perp} p_{\perp}{ }^{2} e^{-p_{\perp} / T}}{\int_{0}^{\infty} d p_{\perp} p_{\perp}{ }^{2} e^{-p_{\perp} / T}} \\
& =e^{-p_{M} / T}\left(\frac{1}{2}\left(\frac{p_{M}}{T}\right)^{2}+\left(\frac{p_{M}}{T}\right)+1\right)
\end{aligned}
$$


This is a monotonically falling function of $\mathrm{p}_{M} / T$; for $\mathrm{p}_{M} / T \sim 1-1.5$ we find that the ratio eq (5.21) varies between .92 and .81 . Hence, inserting eq $(5.22)$ into eq $(5.21)$ we find for $f \sim 2 / 3$

$$
\bar{p}_{q}=p_{q}\left(1-f \frac{p_{q}\left(p_{\perp}>p_{M}\right)}{p_{q}}\right) \cong 0.4 p_{q} \text {. }
$$

Since we used the lower limit on $f$ it is likely that $\bar{p}_{q}$ is even further reduced. Since the quark pressure at $\mu / T \sim 1$ is about twice as large as the glue pressure, $40 \%$ of the total pressure is relieved by radiation and only about $60 \%$ of the internal pressure acts on the surface. Thus, in effect, the time constant relevant for the cooling process through expansions is extended by a factor of almost two. Thus we are led to the conclusion that the expansion contributes only about $20 \%$ to the cooling of the plasma. Even for baryonless plasma, i.e., $\mu=0$, though this effect is somewhat reduced it still relieves $1 / 3$ of the total pressure.

The physical distinction between the cooling by pion radiation and by expansion resides in the reduction of the temperature without a significant increase of the plasma volume. This, of course, has important consequences in the dynamics of the plasma development, and, in particular, eventually on the observable quantities. In particular, cooling by radiation seems to convert more efficiently the internal energy into pions. In an expansion this energy through collective motion becomes additional kinetic energy of the final particles. Hence in the radiation cooling the available entropy is used to create more new particles, i.e., pions, while in the adiabatic expansion it is essentially contained in the kinetic motion. In both instances cooling is approximately adiabatic.

We next discuss the maximally obtainable plasma temperature, neglecting the effect of the cooling by expansion. As already remarked, once the plasma has ignited a fraction of the radiated pions will be swept along by the incoming nucleons and re-enter the plasma. This process introduces a dependence of the loss term on the beam characteristics. Even though this turn-around of the pions does not change the ignition conditions it influences the maximal achievable plasma energy density. Since the thermal radiation is isotropic this returned fraction, $n$, will be of the order $n \lesssim 1 / 2$. To obtain an estimate of this maximum plasma energy density one has to multiply the energy radiation term, eq $(5.15)$, with $(1-n)$ and balance it with the unmodified gain term, eq $(5.9)$. We recall that in the derivation of eq (5.15) a non-degenerate quark-gas has been assumed, and $\mu_{\mathrm{g}} / T$ is expected to be less than 2. As the collision process continues the temperature of the plasma will grow until the nuclear collision terminates or until the temperature has risen to a level at which the pion radiation overwhelms the energy influx. This 
maximum achievable temperature is shown in figure 5.3 for a few choices of the pion turn-around coefficient $n$, as a function of projectile beam energy. In view of high plasma density here we have used $R / \lambda=5, \mu / T=1$. As one can see the maximal temperature achievable in the collision does not depend too sensitively on the choice of the parameters and reaches for $50 \mathrm{GeV}$ a value around $230 \mathrm{MeV}$. Hence, once a plasma has ignited one can expect that a fullfledged quark-gluon plasma event will take place, with energy density reaching 4-5 GeV/ $/ \mathrm{fm}^{3}$. However, we note that underlying this scenario is the requirement that the collisions take place between two quite heavy nuclei.

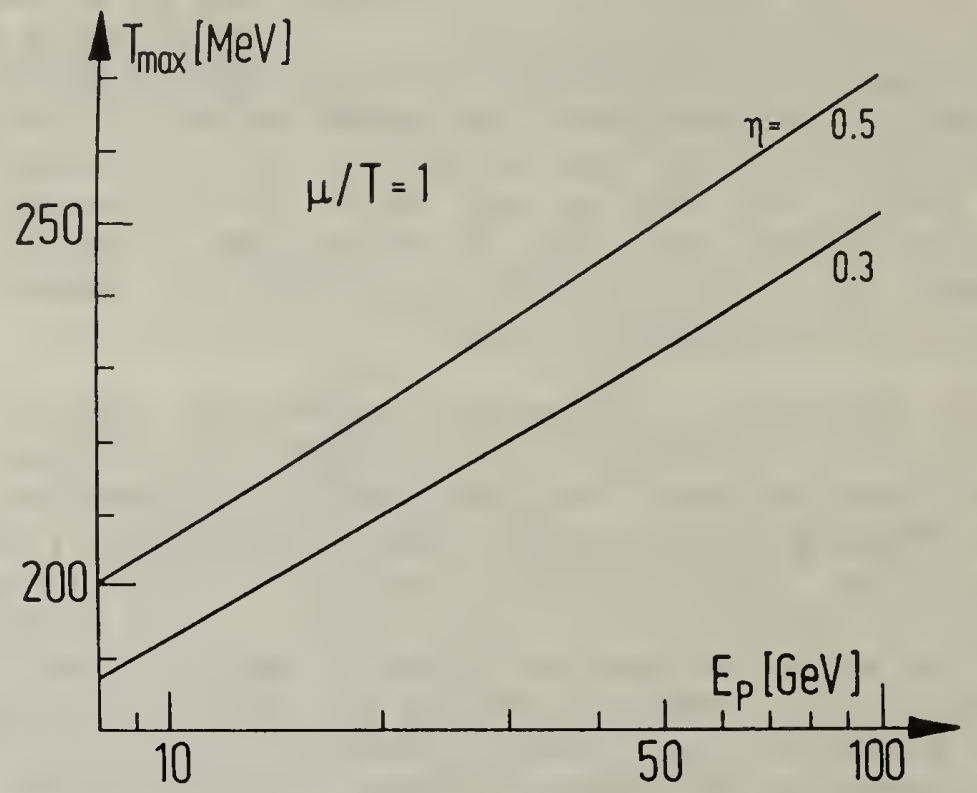

Fig. 5.3 Maximum achievable plasma temperature as function of beam laboratory energy for two values of the pion turn-around coefficient.

Considering experiments at beam

energies 20 - $50 \mathrm{GeV} / \mathrm{A}$ one should recognize that even in symmetric collision, e.g., $\mathrm{Pb}$ on $\mathrm{Pb}$, the plasma will not necessarily always occur at central rapidity. Namely, if the seed occurs relatively late in the collision then even for head-on collisions plasma would be created in a non-symmetric configuration, as illustrated in figure 5.4 which is drawn in the $C M$ frame. In this example the plasma will appear shifted towards the target rapidity. However, the largest and hottest plasma events of necessity will arise at about central rapidity since they must be seeded early in the collision. This circumstance could be utilized in the design of the experiment. We note that this type of dicussion requires event by event analysis.

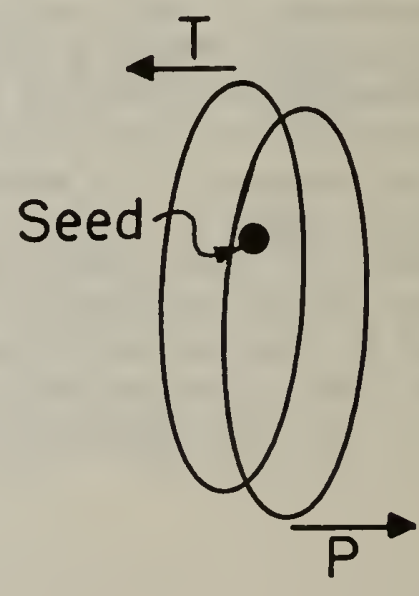

Fig. 5.4 Non-symmetric plasma arising from late seed. 


\section{PHASE TRANSITION OR PERHAPS TRANSFORMATION: HADRONIC GAS TO THE QUARK GLUON PLASMA}

We have described two inherently different descriptions leading to the prediction of a qualitatively similar thermodynamic region for the transition between both phases of hadronic matter. As we shall see in a moment the physics which went into these theoretical approaches requires that this is a first order phase transition. However, of course, we cannot actually deduce the order of the transition in the presented considerations. We record here that recent Monte-Carlo simulations on a lattice show phase coexistence in SU(3) gauge theories which is characteristic of first order phase transitions [6a], [41]. This is contrary to results found in SU(2) simulations [42].

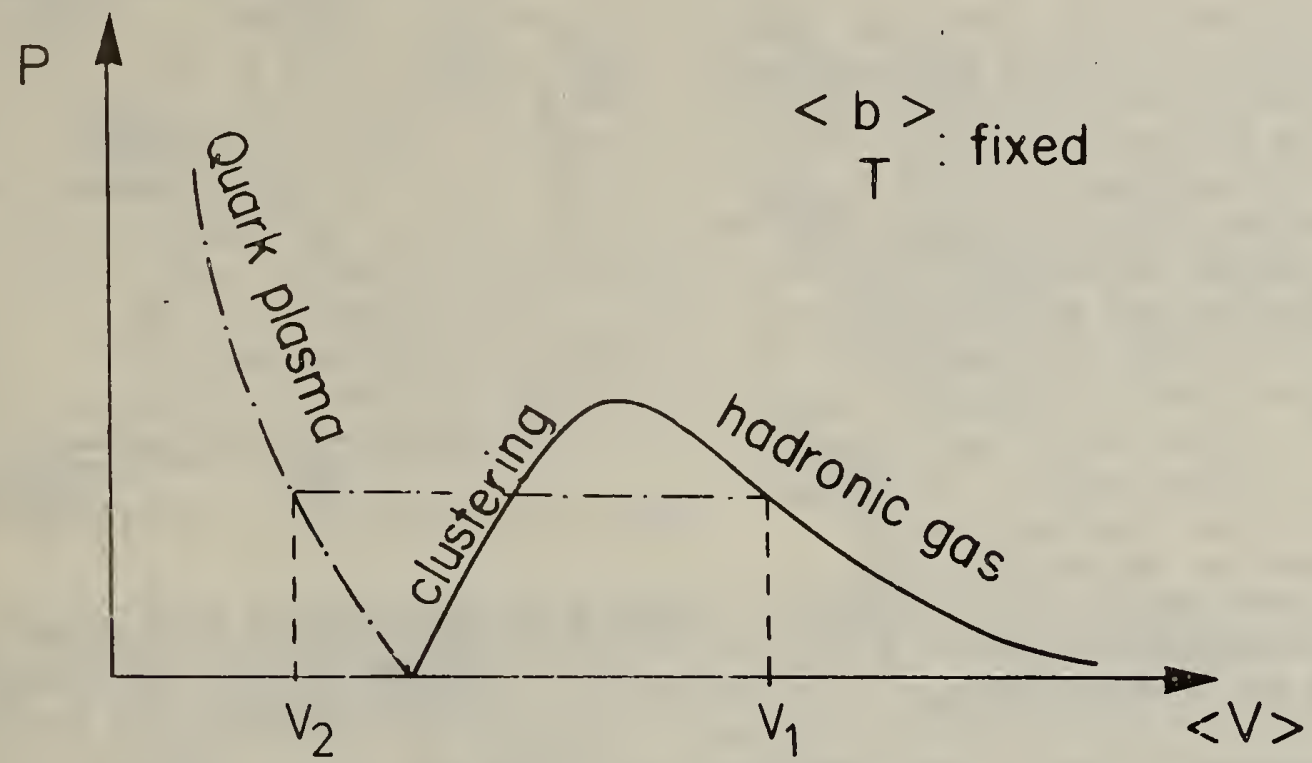

Fig. 6.1 P-V diagram for the gas-plasma first order transition.

Consider the P-V diagram shown in figure 6.1. Here we distinguish three domains. The hadronic gas region is simply a Boltzmann gas where the pressure raises with reduction of the volume. When the internal excitation rises, the individual hadrons begin to cluster. This reduces the increase in the Boltzmann pressure since a smaller number of particles exercises a smaller pressure. In a complete description of the different phases we have to allow for a coexistence of hadrons with the plasma state in the sense that the internal degrees of freedom of each cluster, i.e., quarks and gluons contribute to the total pressure even before the dissolution of individual hadrons. This indeed becomes necessary when the clustering overtakes the compressive effects and the hadronic gas pressure falls to zero as $V$ reaches the proper volume of hadronic matter. At this point the pressure rises again very quickly, since in absence of individual hadrons we now compress only the hadronic constituents. By performing the Maxwell construction between volumes $V_{1}$ and $V_{2}$ as indicated in figure 6.1 we can in part account for the complex process of hadronic compressibility alluded to above. We find this way the most likely path taken by the compressed hadronic gas in a nuclear collision. This discussion shows that in our approach we are straightforwardly led to a first order phase transition [43]. We should remember that on the way out, i.e., during the final step, presumably an expansion of the plasma state, the 
entropy generated and still present in the plasma may require that the

(isolated) plasma state must expand to close to $P=0$, i.e., under-cool

before it can disintegrate into

individual hadrons. This disintegration may be a quite slow process of successive fissions, when color neutrality, i.e., the door to freedom, can no longer be achieved by particle production.

It is interesting to follow the path taken by an isolated quark-gluon plasma fireball in the $\mu-T$ plane, or equivalently in the $v-T$ plane.

Several cases are depicted in figure 6.2. In the Big Bang expansion the cooling shown by the dashed line occurs in a universe in which most of the energy is in the radiation. Hence, we have for the chemical potential $\mu \ll T$. Similarly, the baryon density $v$ is quite small. In normal stellar collapse leading to cold neutron stars we follow the dashdotted line parallel to the $\mu$-resp. $v$-axis. The compression is accompanied by little heating.
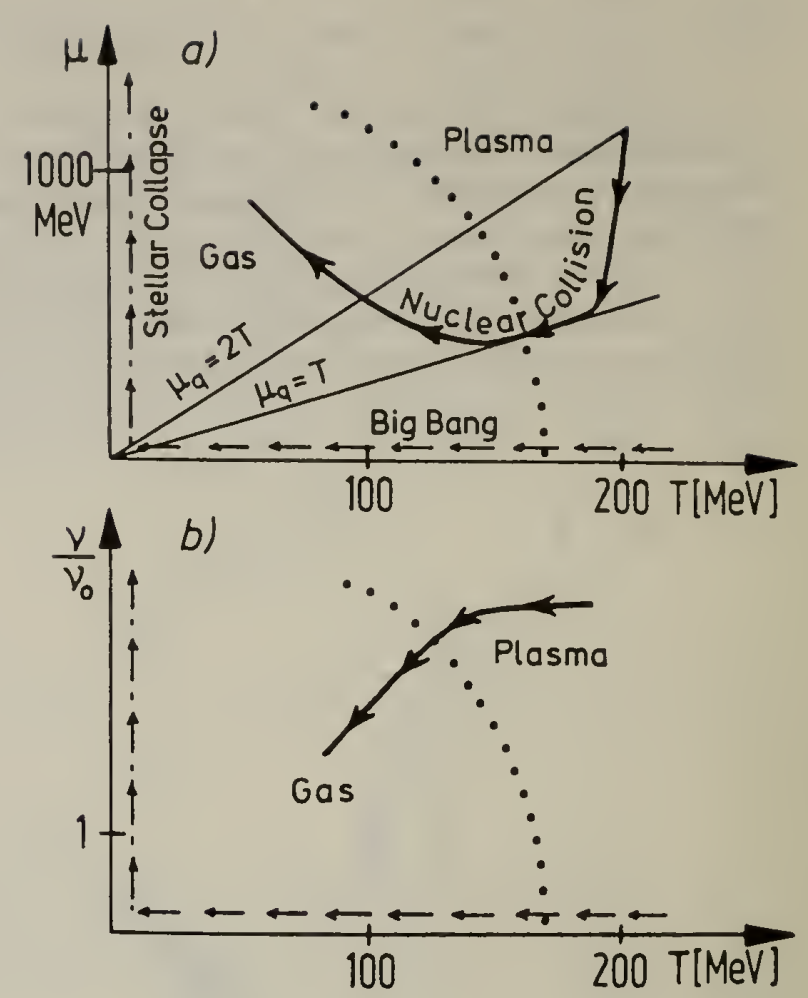

Fig. 6.2 Paths taken in the (a) $\mu-T$ plane and (b) $v-T$ plane by different physical events.

In contrast, in nuclear collisions almost the entire $\mu-T$ and $\nu-T$ can be explored by varying the parameters of the colliding nuclei. We show an example by the full line, and we show only the path corresponding to the cooling of the plasma, i.e., the part of the time evolution after the termination of the nuclear collision. The figure reflects the circumstance that in the beginning of the cooling phase, i.e., for $1-1.5 \times 10 .-23 \mathrm{sec}$, the cooling happens almost exclusively by the mechanism of pion radiation. In terms of our numerical example of section 5 this means that during that time about half of the available energy has been radiated away. More precisely, at $t=1.5 \times$ $10^{-23} \mathrm{sec}, 200 \mathrm{GeV}$ has been radiated, and the initial surface temperature of $180 \mathrm{MeV}$ has decreased to $T=150 \mathrm{MeV}$. This is close to the temperature of the transition to the hadronic phase. Hence a possible, perhaps even likely, scenario is that in which the freezing-out and the expansion happen simultaneously. If that in fact takes place then the expanding hadronic gas will be quite dilute and a hadron will not undergo many scattering events before reaching an asymptotic distance, i.e., before becoming accessible to detection by an experiment. Of course, this would be ideal in that the detected particles then would reflect the chemical composition of the plasma at the freeze-out point. These highly speculative remarks are obviously made in the absence of experimental guidance. A careful study of the hadronization process most certainly remains to be performed. 
Let us now consider the question: is the transition hadronic-gas $\leftrightarrow$ quark-gluon plasma in principle a phase transition or is it only a change in the nature of hadronic matter, not associated with any kind of singularity of the partition function in the limit of infinite volume? The spirit of the theoretical approaches taken here requires a first order transition. However, this conclusion is only preliminary. Contrary arguments can be found [44] by arguing that only a finite number of incompressible hadrons can be fitted into a given volume. Here it turns out that one must very carefully study the meaning of thermodynamical limits (see references [9d] and [45]). Even worse: for compressible individual hadrons we might find a second order phase transition [43]. We see that the theory is highly sensitive at this point. It is only the experiment which will teach us this important aspect of strong interactions. Numerical digital experiments concluded recently [41] allow one to believe that we have been on the right track with our description of the hadronic world. Infortunately we probably will have to wait for some years for the needed experiments in view of the newest emphasis on less relevant research fields that lack the "disadvantage" of being interdisciplinary. As the reader further notes, this is the shortest section of this work - and we hope that as soon as the just mentioned required experimental advances occur, we will review and re-analyze its content, we hope before the end of this century. To encourage these experiments we therefore describe in the next section our current favorite observable.

\section{STRANGENESS IN QUARK-GLUON PLASMA}

We now show in some detail why the strange particle abundances are so helpful [4] in observing the formation and the properties of the quark-gluon plasma. First we note that at a given temperature the quark-gluon plasma will contain an equal number of strange (s) quarks and antistrange ( $\bar{s}$ ) quarks, naturally assuming that the hadronic collision time is much too short to allow for conversion to strangeness of the light flavors by the weak interaction. Thus, assuming chemical and thermal equilibrium in the quark plasma we find the density of the strange quarks to be (two spins and three colors):

$$
s / V=\bar{s} / V=6 \int \frac{d^{3} p}{(2 \pi)^{3}} \frac{1}{\left(e^{\sqrt{p^{2}+m_{s}^{2} / T}}+1\right)} \approx 3 \frac{T m_{s}^{2}}{\pi^{2}} K\left(m_{s} / T\right)
$$

neglecting, for the time being, the QCD perturbative corrections. We recall that the mass of the strange quarks, $m_{s}$, in the perturbative vacuum is believed to be of the order of $140-200 \mathrm{MeV}$ [18]. In eq (7.1) we were able to use the Boltzmann limit since the phase space density of strangeness is not too high. Similarly, there is a certain light antiquark density ( $\bar{q}$ stands for either $\bar{u}$ or $\bar{d})$ :

$$
\bar{q} / V=6 \int \frac{d^{3} p}{(2 \pi)^{3}} \frac{1}{\left(e^{|p| / T+\mu_{q} / T}+1\right)} \approx e^{-\mu_{q} / T} T^{3} \frac{6}{\pi^{2}} .
$$


The chemical potential of quarks surpresses the $\bar{q}$ density. This phenomenon reflects on the chemical equilibrium between $q-\bar{q}$ and the presence of a lightquark density associated with the net baryon number. Alternative but physically equivalent ways to understand this factor are the following two statements:

1) $\bar{q}$ is Fermi-blocked, since in its production the partner q-quark has to go on top of the Fermi sphere $(T+0$ limit);

2) $\bar{q}$ are easily destroyed by the abundant q's when the q-density is large.

We now intend to show that often more $\bar{s}$ quarks are present than antiquarks of either light flavor. Indeed:

$$
\bar{s} / \bar{q}=\frac{1}{2}\left(\frac{m s}{T}\right)^{2} K_{2}\left(\frac{m}{T}\right) e^{\mu / 3 T} .
$$

This ratio is shown in figure 7.1. Thus, we almost always have more $\bar{s}$ than $\bar{q}$ quarks and, in many cases of interest, $\Sigma / \varnothing \sim 5$. For $\mu+0$ there are about as many $\bar{u}$ and $\bar{d}$ quarks as there are $\bar{s}$ quarks at $T \sim m_{s}$.

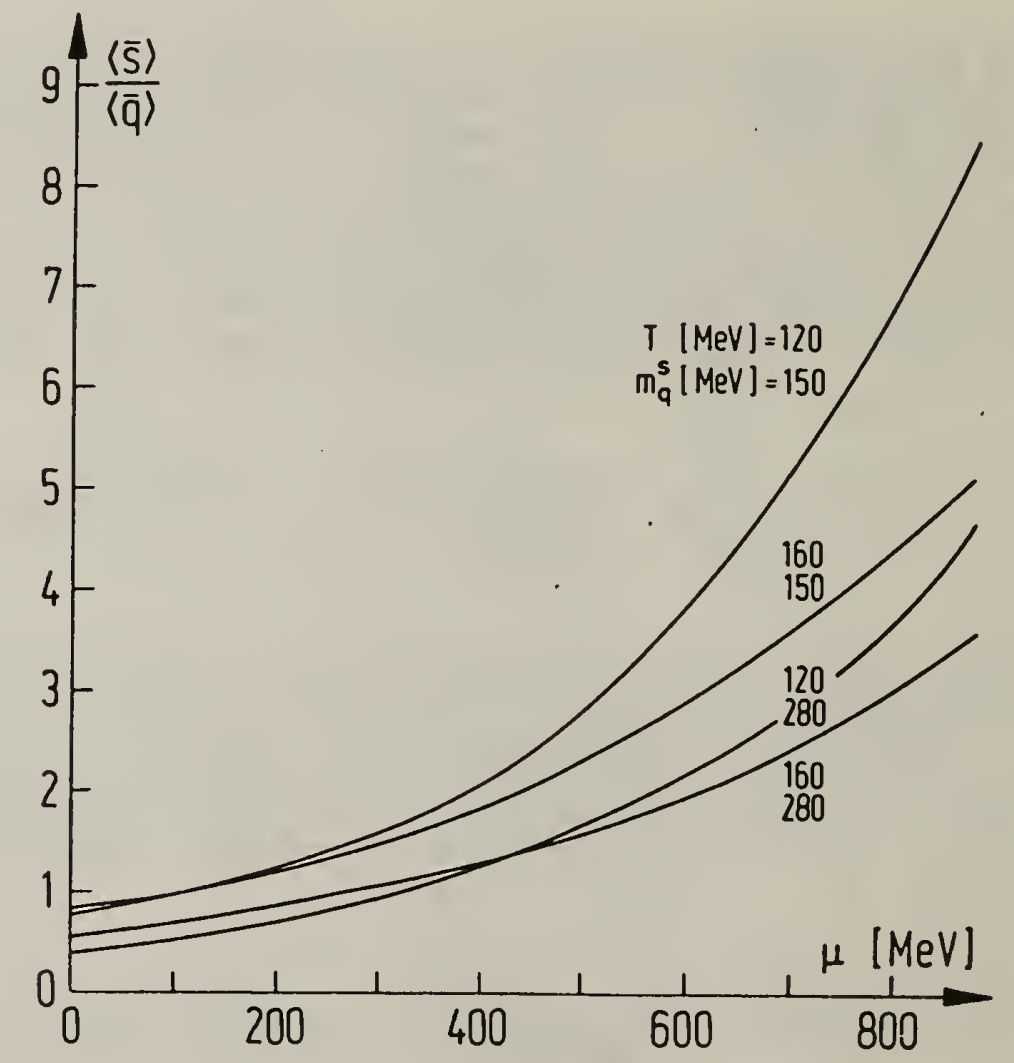

Fig. 7.1 Abundance of strange (or antistrange) quarks relative to the light quark abundance as function of $\mu$ for several choices of the temperature $T$ and of the strange quark mass $m_{s}$. 
When the quark matter dissociates into hadrons some of the numerous $\bar{s}$, instead of being bound in a qs kaon $\left(K^{+}, K, 2\right.$, may enter into a (qqs) or (qडs ) antibaryon and, in particular, a $\bar{\Lambda}, \bar{\Sigma}$, or $\Xi$. The probability for this process seems to be of similar magnitude to the production of $\Lambda, \Sigma$, or $\Xi$ by the quarks present in the plasma. It is particularly noteworthy about the 5 carrying antibaryons that conventionally, i.e., in pp collisions, they can be produced only in direct pair production reactions. This process is suppressed by energy-momentum conservation up to high energies and phase space considerations since the final state has to contain four particles. This leads to the argument that a study of the $\bar{\Lambda}, \bar{\Sigma}, \bar{\Xi}$ in high-energy nuclear collisions could shed light on the early stages of the reaction in which a quark-gluon plasma may be formed.

As is apparent from the above remark, the crucial aspects of the proposal to use strangeness as a signature for the quark-gluon plasma involve:

a) assumption of thermal and chemical equilibrium

b) comparison between results anticipated in both hadronic phases at given $T$ and $\mu$, winere the chemical potential must be determined by other considerations.

We now turn to the discussion of both these points and begin by calculating the abundance of strangeness as function of the lifetime and excitation of the plasma state $[4 b]$.

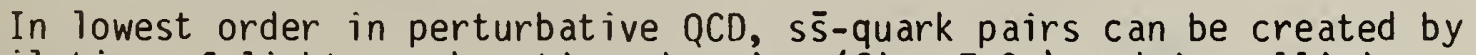
annihilation of light quark-antiquark pairs (fig. 7.2a) and in collisions of two gluons (fig. 7.2b). The averaged total cross sections for these processes were calculated by Combridge [46]. For fixed invariant mass-squared $s=\left(k_{1}+k_{2}\right)^{2}$, where $k_{j}$ are the four-momenta of the incoming particles $\left(w(s)=\left(1-\frac{4 M^{2}}{s}\right)^{1 / 2}\right):$

a)
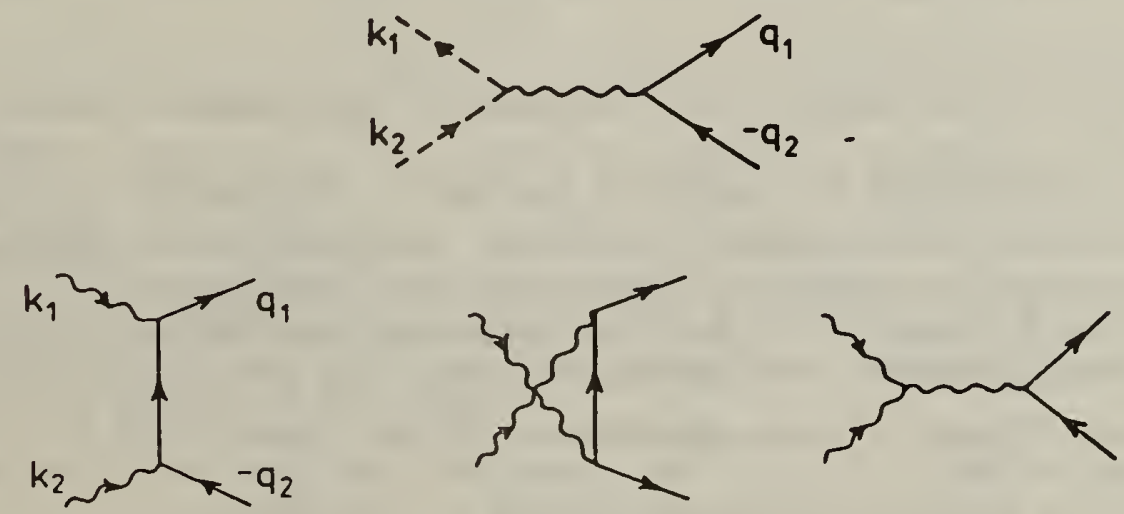

b)

Fig. 7.2 Lowest order QCD diagrams for ss production: a) $q q \rightarrow \mathrm{s} \bar{s} ;$ b) $g g \rightarrow s \bar{s}$. 


$$
\begin{gathered}
\bar{\sigma}_{q \bar{q}+s \bar{s}}=\frac{8 \pi \alpha_{s}^{2}}{27 s}\left(1+\frac{2 M^{2}}{s}\right) w(s) \\
\bar{\sigma}_{g g+s \bar{s}}=\frac{2 \pi \alpha_{s}^{2}}{3 s}\left[\left(1+\frac{4 M^{2}}{s}+\frac{M^{4}}{s^{2}}\right) t^{2} a n h^{-1} w(s)-\left(\frac{7}{8}+\frac{31}{8} \frac{M^{2}}{s}\right) w(s)\right] .
\end{gathered}
$$

For the mass of the strange quark we will assume, a) the value [10] fitted within the MIT bag model: $m_{s}=280 \mathrm{MeV}$ and, b) the typical value [18] found in the study of quark currents: $m_{s}=150 \mathrm{MeV}$. When discussing light quark production below we will use $\mathrm{m}_{\mathrm{g}}=\mathrm{s}_{15} \mathrm{MeV}$. The effective QCD coupling constant $\alpha_{s}=g^{2} / 4 \pi$ is an average over space-like and time-like domains of momentum transfers in the reactions shown in figure 7.2 as discussed in section 3 . We use: (a) $\alpha_{s}=2.2$, the value consistent with $m_{s}=280 \mathrm{MeV}$ in the MIT bag model, and (b) the value $\alpha_{s}=0.6$, expected at the involved momentum transfers. We consider the choice (b) of the parameters to be realistic and consistent with the spirit of this work. The choice (a) is used as a reference; even when $m_{s}=280 \mathrm{MeV}$ it shows that the chemical equilibrium will be reached.

Given the averaged cross sections it is easy to calculate the rate of events per unit time, summed over all final and initial states:

$$
\begin{aligned}
\frac{d N}{d t}=\int d^{3} x \int \frac{d^{3} k_{1}}{(2 \pi)^{3}\left|k_{1}\right|} \sum_{i} \rho_{j}\left(k_{1}, x\right) \\
\\
\qquad \frac{d^{3} k_{2}}{(2 \pi)^{3}\left|k_{2}\right|} \sum_{i} \rho_{j}\left(k_{2}, x\right) \int_{4 M^{2}}^{\infty} d s \delta\left(s-\left(k_{1}+k_{2}\right)^{2}\right) k_{1}^{\mu} k_{2 \mu} \bar{\sigma}(s) .
\end{aligned}
$$

The sum over initial states involves the discrete quantum numbers $i$ (color, spin, etc.) over which eq (7.5) was averaged. The factor $\frac{k_{1} \cdot k_{2}}{\left|k_{1}\right|\left|k_{2}\right|}$ is the relative velocity for massless particles, and we have introduced a dummy integration over $s$ in order to facilitate the calculations. We now replace the phase space densities $\rho_{j}(k, x)$ by momentum distributions $f_{g}(k), f_{g}(k)$, $f(k)$ of gluons, quarks, and antiquarks that can still have a parametric $x$-dependence through a space-dependence of the temperature $T=T(x)$ and the chemical potential $\mu=\mu(x)$. The invariant rate per unit time and volume for the elementary processes shown in figure 7.2 is then: 


$$
\begin{aligned}
A=\frac{d N}{d t d^{3} x} & =\frac{1}{2} \int_{4 M^{2}}^{\infty} s d s \delta\left(s-\left(k_{1}+k_{2}\right)^{2}\right) \int \frac{d^{3} k_{1}}{(2 \pi)^{3}\left|k_{1}\right|} \int \frac{d^{3} k_{2}}{(2 \pi)^{3}\left|k_{2}\right|} \\
& \times\left\{(2 \times 8)^{2} f_{g}\left(k_{1}\right) f_{g}\left(k_{2}\right) \bar{\sigma}_{g g \rightarrow s \bar{s}}(s)\right. \\
& \left.+2 \times(2 \times 3)^{2} f_{q}\left(k_{1}\right) f_{\bar{q}}\left(k_{2}\right) \bar{\sigma}_{q \bar{q}+s \bar{s}}(s)\right\},
\end{aligned}
$$

where the numerical factors count the spin, color and isospin degrees of freedom.

Assuming that in the rest frame of the plasma the distribution functions $f$ depend only on the absolute value of the momentum, $|k|=k_{0} \equiv k$, we can evaluate the angular integrals in eq (7.7):

$$
\begin{aligned}
& A=\frac{8}{\pi^{4}} \int_{4 M^{2}}^{\infty} s d s \bar{\sigma} g \rightarrow s \bar{s}\left[\int_{0}^{\infty} d k_{1} \int_{0}^{\infty} d k_{2} \theta\left(4 k_{1} k_{2}-s\right) f_{g}\left(k_{1}\right) f_{g}\left(k_{2}\right)\right] \\
& +\frac{9}{4 \pi^{4}} \int_{4 M^{2}}^{\infty} s d s \bar{\sigma} q \bar{q}+s \bar{s}-\left[\int_{0}^{\infty} d k_{1} \int_{0}^{\infty} d k_{2} \Theta\left(4 k_{1} k_{2}-s\right) f_{q}\left(k_{1}\right) f_{\bar{q}}\left(k_{2}\right)\right],
\end{aligned}
$$

where the step function $\theta$ requires that $k_{1} k_{2} \geqslant \frac{5}{4}>M^{2}$. We now turn to the discussion of the momentum distribution and related questions. We note that the anticipated lifetime of the plasma created in nuclear collisions, as discussed in section 5 , is about $6 \mathrm{fm} / \mathrm{c}=2 \times 10^{-23} \mathrm{sec}$. After this time the high internal excitation will most likely have dissipated to below the energy density required for the global restoration of the perturbative QCD vacuum state. We recall again that the transition between the hadronic and the quark-gluon phase is expected at an energy density of approximately 0.6 $1 \mathrm{GeV} / \mathrm{fm}^{3}$. Under these conditions we note that each perturbative quantum (light quark, gluon) in the plasma state will rescatter several times during the lifetime of the plasma. Hence the momentum distribution functions $f(p)$ can be approximated by the statistical Bose or Fermi distribution functions, regardless of the shortness of time:

$$
f_{g}(p) \approx\left(e^{\beta \cdot p}-1\right)^{-1}, \quad \text { (gluons) }
$$




$$
f_{q / \bar{q}}(p)-\left(e^{\beta \cdot p} \lambda^{ \pm}+1\right)-1, \quad \text { (quarks-antiquarks) }
$$

where again $\beta \cdot p=\beta_{0}|\vec{p}|-\vec{\beta} \cdot \vec{p}$ for massless particles, $(\beta \cdot \beta)^{-1 / 2}=T$ is the temperature and $\lambda^{ \pm}$is the baryon number (antibaryon number) fugacity. In the rest frame of the plasma, $\beta \cdot p=|\vec{p}| / T$. The distributions $(7.9)$ can be taken seriously only for $|\vec{p}|$ not very much larger than $T$; to populate the high energy tail of the distributions too many collisions are required for which there may not be enough time during the lifetime of the plasma. Furthermore, we note that while in each individual nuclear collision the momentum distribution may vary, the ensemble of many collisions may lead to better statistical distributions.

Finally, consider the values of the fugacities $\lambda^{ \pm}$in eq (7.9b). As we will show the $g g \rightarrow q \bar{q}$ reaction time is much shorter than that for $q \bar{q} \rightarrow \mathrm{s} \bar{s}$ production since the light quark masses are only of the order of $\sim 15 \mathrm{MeV}$. Consequently we may assume chemical equilibrium between $q$ and $q$, $\underline{i} . \underline{e}$.,

$$
\lambda^{+}=\frac{1}{\lambda^{-}}=e^{-\mu_{q} / T}, \mu=3 \mu_{q}
$$

and the baryon density is given by eq $(4.35)$ omitting for the present the $O(\alpha)$ corrections, i.e..;

$$
v\left(T, \mu_{q}\right)=\frac{2}{3 \pi^{2}}\left(\mu_{q}{ }^{3}+\mu_{q}(\pi T)^{2}\right) .
$$

We note that since gluons dominate the ss production in the plasma state, the conditions at the phase transition, such as abundance of $q$ and $q$, will not matter for the $\mathbf{s} \bar{s}$ abundances at times comparable to the lifetime of the plasma.

We now return to the evaluation of the rate integrals, eq (7.8). In the glue part of the rate $A$, eq $(7.8)$, the $k_{1}, k_{2}$ integral can be carried out exactly by expanding the Bose function in a power series in $\exp (-k / T)$ :

$$
\left.A_{g}=\frac{8}{\pi^{4}} T \int_{4 M^{2}}^{\infty} d s s^{3 / 2} \bar{\sigma}_{g g+s \bar{s}}(s) \sum_{n, n^{\prime}=1}^{\infty}\left(n n^{\prime}\right)-1 / 2 K_{1}\left(\frac{\left(n n^{\prime} s\right.}{T}\right)^{1 / 2}\right) .
$$

In the quark contribution an analytic treatment of the Fermi function is not feasible and the integrals must be evaluated numerically. It is found that the gluon contribution, eq $(7.11)$, dominates the rate $A$. For $T / M \gtrsim 1$ we find: 


$$
A \approx A_{g}=\frac{7}{3 \pi^{2}} \alpha_{s}{ }^{2} M T^{3} e^{-2 M / T}\left(1+\frac{51}{14} \frac{T}{M}+\ldots\right)
$$

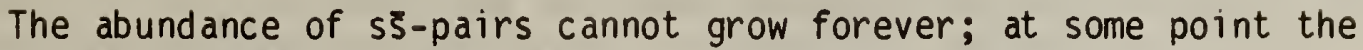
ss-annhilation reaction will deplete the strange quark population. It is important to appreciate that the sडs-pair annihilations may not proceed via the two gluon channel, but instead occasionally through $Y G$ final states [47]. The noteworthy feature of such a reaction is the production of relatively high energetic $\gamma^{\prime} s$ at an energy of about $700-900 \mathrm{MeV}(T=160 \mathrm{MeV}$ ) stimulated by coherent glue emission. These $\gamma^{\prime} s$ will leave the plasma without further interactions and provide an independent confirmation of the s-abundance in the plasma.

The loss term of the strangeness population is proportional to the square of the density $n_{s}$ of strange and antistrange quarks. With $n_{s}(\infty)$ being the saturation density at large times, the following differential equation determines $n_{s}$ as function of time:

$$
\frac{d n_{s}}{d t} \approx A\left[1-\left(n_{s}(t) / n_{s}(\infty)\right)^{2}\right] .
$$

We note that eq (7.13) in principle should also include a term linear in $n_{s}(t)$. Namely, when the plasma density is sufficiently high the produced strange quarks have difficulty to quickly get away from each other. With a scattering length of the order of $1 / 3 \mathrm{fm}$ in extreme cases one has to consider diffusion rather than free motion. Hence in this limiting case we have always a š̄ pair in a given unit volume, leading to [48]

$$
\frac{d n_{s}}{d t} \approx A\left(1-n_{s}(t) / n_{S}(\infty)\right) .
$$

The solutions of eq (7.13) are, respectively

$$
\begin{aligned}
& n_{s}(t)=n_{s}(\infty) \tanh (t / \tau) \\
& n_{s}(t)=n_{s}(\infty)\left(1-e^{-t / \tau}\right)
\end{aligned}
$$

with

$$
\tau=n_{s}(\infty) / A
$$


Both solutions are monotonically rising saturating functions with similar behavior, controlled by the characteristic time constant $\tau$. In a thermally equilibrated plasma the asymptotic strangeness density, $n_{s}(\infty)$, is that of a chemically unconstrained relativistic Fermi gas $(\lambda=1)$ :

$$
n_{S}(\infty)=\frac{2 \times 3}{2 \pi^{2}} T M^{2} \sum_{n=1}^{\infty} \frac{(-)^{n-1}}{n} K_{2}(n M / T),
$$

We find for the relaxation time (7.14c) from eq (7.12), (7.15)

$$
\tau \approx \tau_{g}=\frac{9}{7}\left(\frac{\pi}{2}\right)^{1 / 2} \alpha_{S}^{-2} M^{1 / 2} T^{-3 / 2} e^{M / T}\left(1+\frac{99}{56} \frac{T}{M}+\ldots\right)^{-1}
$$

which falls rapidly with increasing temperature.

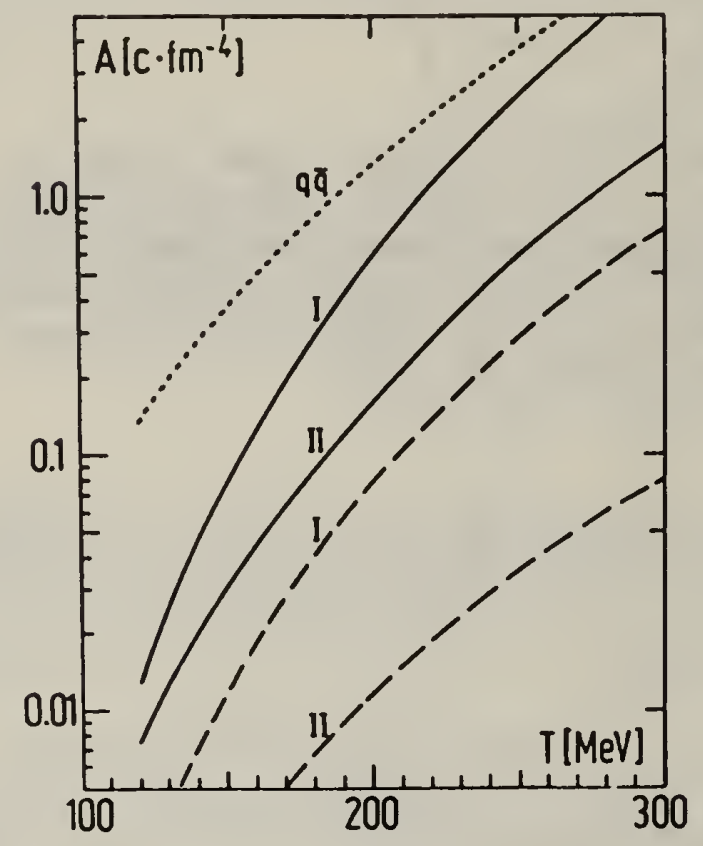

(a)

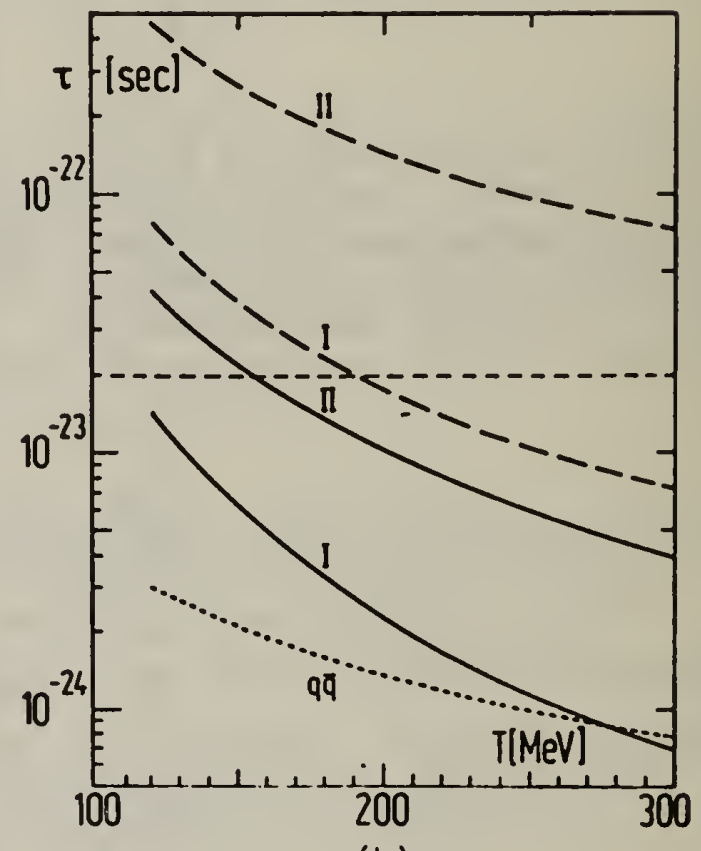

(b)

Fig. 7.3 Chemical relaxation times as functions of the temperature $T$. Full lines: $q \bar{q} \rightarrow$ s5 and $g g+s 5$; dashed 1 ines $q q \rightarrow s 5$; dotted lines $g g \rightarrow q q\left(m_{g}=15 \mathrm{MeV}\right)$. Curves marked I are for $\alpha_{\mathrm{S}}=2.2$ and $\mathrm{m}_{\mathrm{S}}=280 \mathrm{MeV}$, those marked II are for $\alpha_{\mathrm{s}}=0.6$ and $\mathrm{m}_{\mathrm{s}}=150 \mathrm{MeV}:$ a) rates $A$; b) time constants $\tau$.

We now discuss the numerical results for the rates, time constants, and the expected strangeness abundance. In figure 7.3a we compare the rates for strangeness production by the processes depicted in figure 7.2 for the two different choices of parameters discussed above, after eq $(7.5)$. The rate for $q q \rightarrow$ ss alone (shown separately) contributes less than 10 percent to the total 
rate. In figure $7.3 \mathrm{~b}$ we show the corresponding characteristic relaxation times toward chemical equilibrium, $\tau$, defined in eq (7.14). While our results for strangeness production by light quarks agree only in order of magnitude with those of Biro and Zimanyi [49] owing to the difference in the chosen values of the parameters, it is obvious from our results that gluonic strangeness production, which was not discussed initially by these authors [50], is the dominant process. If we compare the time constant $\tau$ with the estimated lifetime of the plasma state we find that the strangeness abundance will be chemically saturated for temperatures of $160 \mathrm{MeV}$ and above, i.e., for an energy density above $1 \mathrm{GeV} / \mathrm{fm}^{3}$. We note that $\tau$ is quite sensitive to the choice of the strange quark mass parameter and the coupling constant $\alpha_{s}$ which must, however, be chosen consistently. A measure of the uncertainty associated with the choice of parameters is illustrated by the difference between our results for the two parameter sets taken here.

Also included in figures $7.3 a$, and $7.3 b$ are our results for gluon conversion into light quark-antiquark pairs. The shortness of $\tau$ for this process indicates that gluons and light quarks reach chemical equilibrium during the beginning stage of the plasma state, even if the quark/antiquark, i.e., baryon/meson ratio was quite different in the prior hadronic compression phàse.

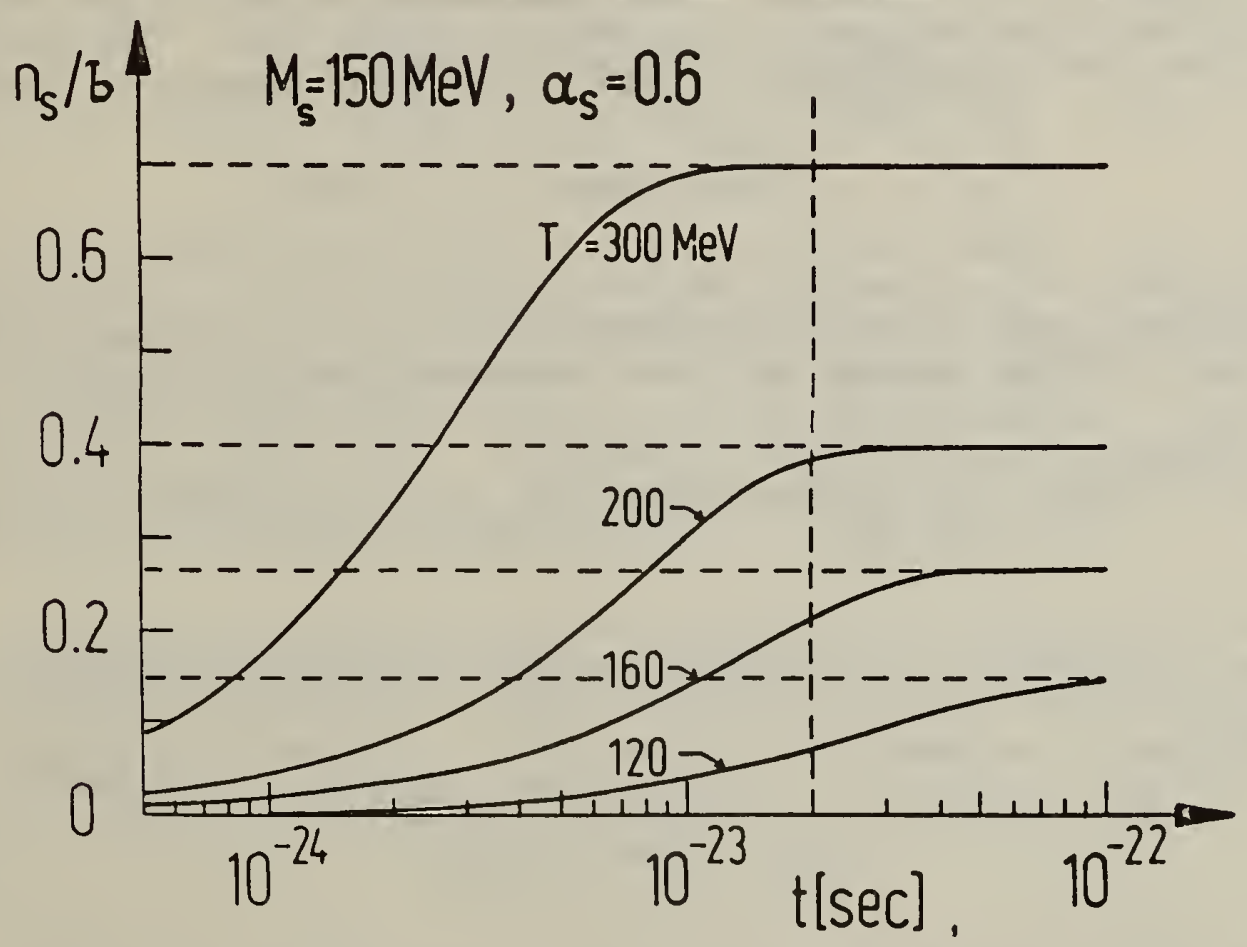

Fig. 7.4 Time evolution of the relative strange quark to baryon number abundance in the plasma for various temperatures T. $m_{s}=150 \mathrm{MeV}$, $\alpha_{s}=0.6$.

The evolution of the density of strange quarks, eq (7.14), relative to the baryon number content of the plasma state, is shown in figure 7.4 for various temperatures. The saturation of the abundance is clearly visible for $T>160 \mathrm{MeV}$. To obtain the experimentally accessible abundance of strange 
quarks, the corresponding values reached after the typical lifetime of the plasma state, $2 \times 10^{-23} \mathrm{sec}, \mathrm{c}$ an be read off in figure 7.4 as a function of temperature. The strangeness abundance shows a pronounced threshold behavior at $T \sim 120-160 \mathrm{MeV}$.

We thus conclude that strangeness abundance saturates in a sufficiently excited quark-gluon plasma with $\mathrm{T}>160 \mathrm{MeV}, \varepsilon>1 \mathrm{GeV} / \mathrm{fm}^{3}$, al lowing strangeness to be an important observable. We hence turn to the study of the strangeness in normal nuclear matter in order to gain insight into the relevance of strangeness as a characteristic signature of the quark-gluon plasma.

To this end we must first establish $[4 c, 51]$ the relevant relative strange particle rates originating from highly excited matter consisting of individual hadrons, the hadronic gas phase. The main hypothes is which allows us to simplify the situation is to postulate the resonance dominance of hadron-hadron interactions (see section 2). In this case the hadronic gas phase is practically a superposition of an infinity of different hadronic gases and all information about the interaction is hidden in the mass spectrum $\tau\left(m^{2}, b\right)$ which describes the number of hadrons of baryon number $b$ in a mass interval $\mathrm{dm}^{2}$. When considering strangeness carrying particles, all we then need to include is the influence of the nonstrange hadrons in the baryon chemical potential established by the nonstrange particles. The total partition function is approximately additive in these degrees of freedom:

$$
\ln Z=\ln Z^{\text {nonstrange }}+\ln Z^{\text {strange }}
$$

For our purposes i.e., in order to determine the particle abundances it is sufficient to list the strange particles separately and we find

$$
\begin{aligned}
& \text { en } z^{\text {strange }}\left(T, V, \lambda_{s}, \lambda_{q}\right)=C\left\{2 W\left(x_{k}\right)\left[\lambda_{s} \lambda_{q}^{-1}+\lambda_{s}^{-1} \lambda_{q}\right]\right. \\
& \left.+2\left[W\left(x_{\Lambda}\right)+3 W\left(x_{\Sigma}\right)\right]\left[\lambda_{S} \lambda_{q}^{2}+\lambda_{S}^{-1} \lambda_{q}^{-2}\right]\right\} \\
& W\left(x_{i}\right)=\left(\frac{m_{i}}{T}\right)^{2} k_{2}\left(\frac{m_{i}}{T}\right) .
\end{aligned}
$$

We have $C=V T^{3} / 2 \pi^{2}$ for a fully equilibrated state. The case of chemical nonequilibrium can be effectively taken care of by using smaller values of $C$. As the strangeness-exchange cross sections are very large, strangeness will be always distributed among all particles in (7.18a) according to the values of the fugacities $\lambda_{\mathrm{g}}=\lambda_{\mathrm{B}} 1 / 3$ and $\lambda_{S}$. Hence we can speak of relative strangeness chemical equilibrium, see below. We neglected to write down quantum statistics corrections as well as the multi-strange particles, $\Xi$ and $\Omega$, as our considerations remain valid in this simple approximation [51]. Interactions are effectively included through explicit reference to the baryon 
number content of the strange particles as just discussed. Nonstrange hadrons influence the strange fraction by establishing the value of $\lambda_{q}$ at the given temperature and baryon density.

The fugacities $\lambda_{s}$ and $\lambda_{g}$ as introduced here control the strangeness and the baryon number, respectively. While $\lambda_{s}$ counts the strange quark content the up- and down-quark content is counted by $\lambda_{q}=\lambda_{B} 1 / 3$.

Using the partition function eq (7.18a) and (7.18b) we calculate for given $\mu_{B}, T$, and $V$ the mean strangeness by evaluating

$$
\left\langle n_{s}-n_{\bar{s}}\right\rangle=\lambda_{S} \frac{\partial}{\partial \lambda_{S}} \text { en } Z^{\text {strange }}\left(T, V, \lambda_{S}, \lambda_{q}\right) \text {, }
$$

which is the difference between strange and anti-strange components. This expression must be equal to zero due to the fact that the strangeness is a conserved quantum number with respect to strong interactions. From this condition we get:

$$
\lambda_{S}=\lambda_{q} \mid \frac{W\left(x_{k}\right)+\lambda_{B}^{-1}\left[W\left(x_{\Lambda}\right)+3 W\left(x_{k}\right)\right]+\lambda_{B}\left[W\left(x_{\Lambda}\right)+3 W\left(x_{\Sigma}\right)\right]}{\mid l / 2} \equiv \lambda_{q} \gamma .
$$

We notice a strong dependence of $\gamma$ on the baryon number. For 1 arge $\mu_{B}$ the term with $\lambda_{\mathrm{B}}{ }^{-1}$ will tend to zero and the term with $\lambda_{\mathrm{B}}$ will dominate the expression for $\lambda_{S}$ and $\gamma$. As a consequence the particles with fugacity $\lambda_{S}$ and strangeness $S=-1$ (note that by convention strange quarks $s$ carry $S=-1$, while strange antiquarks $S$ carry $S=1$ ) are suppressed by a factor $\gamma$ which is always smaller than unity. Conversely, the production of particles which carry the strangeness $S=+1$ will be favored by $\gamma^{-1}$. This is the consequence of the presence of nuclear matter; for $\mu=0$ we find $\gamma=1$.

In order to calculate the mean abundance of strange particles we must introduce for each species its own fugacity which subsequently must be set equal to unity since all different strange particles are in mutual chemical equilibrium by assumption. This assumption is made as a consequence of the Targe strangeness exchange cross sections, in reactions such as

$$
N+K \leftrightarrow Y+\pi
$$

where $Y$ stands for a hyperon $\Lambda, \Sigma$. These are much 1 arger then the strangeness production cross sections, such as

$$
N+N+N+\Lambda+K
$$

or even 


$$
\pi+N+\Sigma+K
$$

when considered at moderate temperatures (energy threshold $>500 \mathrm{MeV}$ ). Hence in nuclear collisions the mutual chemical equilibrium, that is, a proper distribution of strangness among the strange hadrons, most likely will be achieved. By studying the relative yields we can exploit this fact and eliminate the absolute normalization, $C$, cf., eq (7.18) from our considerations. We recall that the value of $C$ is uncertain for several reasons: (i) $V$ is unknown, ( $i i) T^{3}$ is strongly $T(t, r)$-dependent, and ( $\left.i i i\right)$ most importantly, the absolute normalization assumes chemical saturation which is not achieved owing to the shortness of the collision. Indeed we have (cf., eq (7.3))

$$
\frac{d C}{d t}=A_{H}\left(1-C(t)^{2} / C^{2}(\infty)\right),
$$

and the time constant $\tau_{H}=C(\infty) / A_{H}$ for strangness production in nuclear matter can be estimated to be 10-21 sec. [52]. The generation of strangeness is most likely driven by reaction (7.21c). Thus $C$ does not reach $C(\infty)$ in plasma-less nuclear collisions. If the plasma state is formed, then the relevant $C>C(\infty)$.

We now compute the relative strangness abundances. Using eq (7.20) we find from eq (7.18) the grand cononical partition sum for zero average strangeness:

$$
\begin{aligned}
\text { en } z_{0}^{\text {strange }} & =C\left\{2 W\left(x_{K}\right)\left[\gamma \lambda_{K}+\gamma^{-1} \lambda_{R}\right]+2 W\left(x_{\Lambda}\right)\left[\gamma \lambda_{B \Lambda} \lambda_{\Lambda}+\gamma^{-1} \lambda_{B}^{-1} \lambda_{\bar{\Lambda}}\right]\right. \\
& \left.+6 W\left(x_{\Sigma}\right)\left[\gamma \lambda_{B \Sigma}{ }_{B}+\gamma_{B}^{-1} \lambda_{\bar{\Sigma}}^{-1} \lambda_{\bar{\Sigma}}\right]\right\} .
\end{aligned}
$$

The strange particle multiplicities follow from $(i=K, \bar{k}, \Lambda, \bar{\Lambda}, \Sigma, \bar{\Sigma})$ :

$$
\left\langle n_{i}\right\rangle=\lambda_{i} \frac{\partial}{\partial \lambda_{i}} \text { ln }\left.z_{0}^{\text {strange }}\right|_{\lambda_{i}=1} \text {. }
$$

Explicitly we find

$$
\begin{aligned}
& \left\langle n_{K^{ \pm}}>=C \gamma^{\mp 1} W\left(x_{K}\right)\right. \\
& \left\langle n_{\Lambda / \Lambda}>=C \gamma^{ \pm 1} W\left(x_{\Lambda}\right) e^{ \pm \mu_{B} / T}\right.
\end{aligned}
$$


and hence the ratio $\left\langle n_{K^{+}}\right\rangle \mid\left\langle n_{K_{-}}\right\rangle=\gamma^{-2}$. This is shown in figure 7.5 as function of the baryo-chemical potential $\mu_{B}$ for several temperatures.

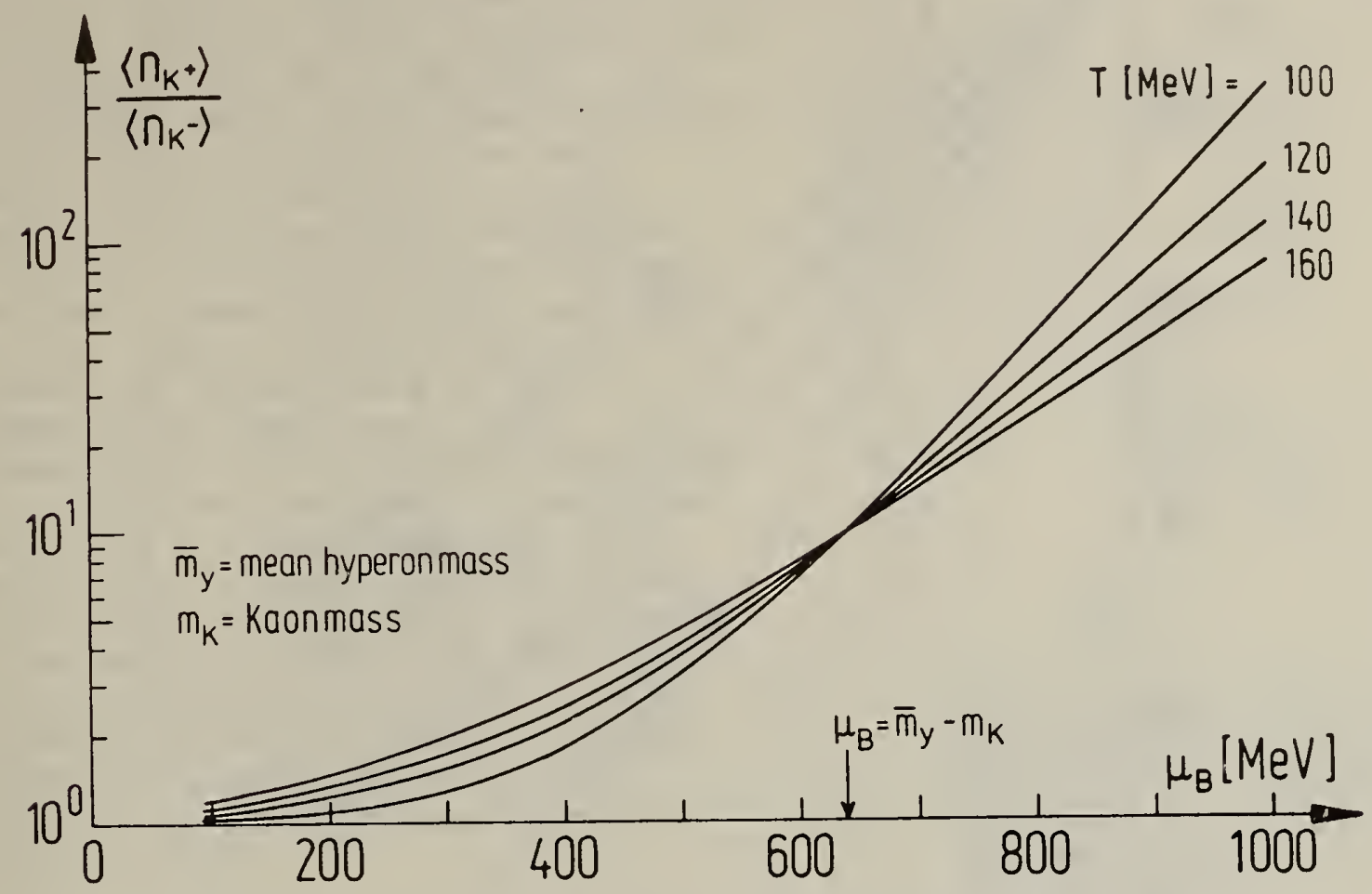

Fig. 7.5 The ratio $\left\langle n_{K^{+}}\right\rangle\left\langle n_{K^{-}}\right\rangle=\gamma^{-2}$ as a function of the baryo-chemical potential for several temperatures.

We note that this particular particle ratio is a good measure of the baryon chemical potential in the hadronic gas phase, provided that the temperatures are approximately known. The mechanism for this process is: the strangeness exchange reaction (eq (7.21a)) tilts to the left $\left(K^{-}\right)$or the right (abundance $\gamma \sim K^{+}$) depending on the value of the baryo-chemical potential.

We turn our further interest to the rarest of all singly strange particles, and show in figure 7.6 the ratio $\left\langle n_{-}\right\rangle /\langle n\rangle$. We notice an expected suppression of $\bar{\Lambda}$ due to the baryo-chemical potential as well as the strangeness chemistry. This ratio exhibits both a strong temperature and $\mu_{B}$ dependence. The remarkably small abundance of $\bar{\Lambda}, e . g ., 10^{-4} \Lambda$, under conditions likely to be reached in an experiment at the end of the dissociation phase $\left(T \sim 120-180 \mathrm{MeV}, \mu_{B} \sim(4-6) T\right)$ is characteristic of the nuclear nature of the hot hadronic matter phase. Our estimates for the quark-gluon plasma based on flavor content are two to three orders of magnitude higher. One may observe that the formation of $\pi$ in nuclear matter will probably be even much less than shown here since $\pi$ will be much further away from the equilibrium abundance than $\Lambda$ 's. Hence the ratio of figure 7.6 may be viewed as an upper limit for the case of hot nuclear matter. 


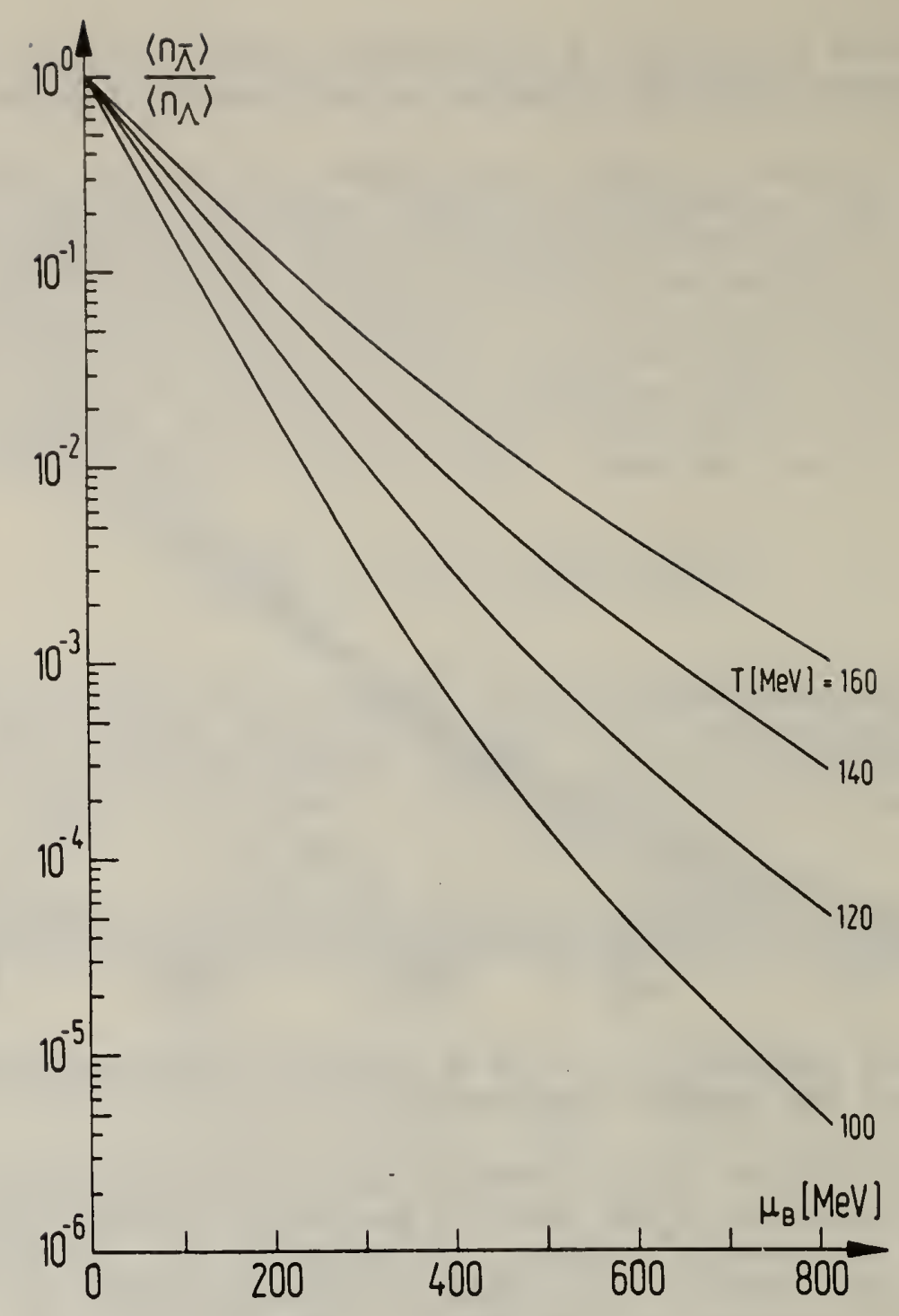

Fig. 7.6 The ratio $\left\langle n_{-}\right\rangle /\langle n\rangle$ as a function of $\mu_{B}$ for several temperatures as an upper limit for $\bar{\Lambda}$ abundance in the hadronic gas phase.

We have already shown that the strangeness abundance is chemically equilibrated in the quark-gluon plasma phase and indicated that this is not the case in the hadronic gas phase. We now further note that even assuming, probably much too optimistically, absolute chemical equilibrium in the gas phase, we find 3 to 5 times more strangness in the plasma at comparable thermodynamic circumstances, i.e., equal $\mu, T$. This is shown in figure 7.7 as function of $\mu$ at some selected values of $T$ and $m_{S}$, where the conversion from $\mu$ as a variable to baryon density has been done using perturbative QCD. Thus the simplest of all observations pointing to the quark-gluon plasma is the measurement of an anomolously high strange particle abundance as function of $C M$ energy in the colliding nuclei, i.e., preferably at high $p_{1}$. Furthermore we have argued that $\bar{\Lambda}$ are strongly suppressed in the nuclear gas. Thus an anomalous yield of $\bar{\Lambda}^{\prime} \mathrm{s}$ is an even more characteristic observable of the plasma. 
More speculative is the observation that strangeness may cluster in the dilute plasma to form strangeness impurities such as $5 \overline{3}$, $\mathbf{5} 5 \overline{5}$, etc., owing to the attractive QCD-Coulomb interaction. Although the ss state would be more bound than $\overline{55}$ or ss state, its statistical weight associated with the color selection factors is so much smaller that we believe the latter state to be the dominant strangeness cluster. This coagulation of strangeness can proceed even further with $\mathbf{s} \bar{s}$ (or sss) states (free $\Omega^{\prime} s$ ) being formed. It is very hard to make a quantitative prediction of this process, but clearly one must look out to measure the abundances of such $r$ are baryons as $\bar{\Omega}$, possibly on event by event basis. However we observe that hadrochemical calculations, i,e., those in hot nuclear matter, along the lines outlined above eq (7.18) also lead to anomalous abundances of multistrange baryons [53] quite similar to the results of a recent hyperon beam experiment [54].

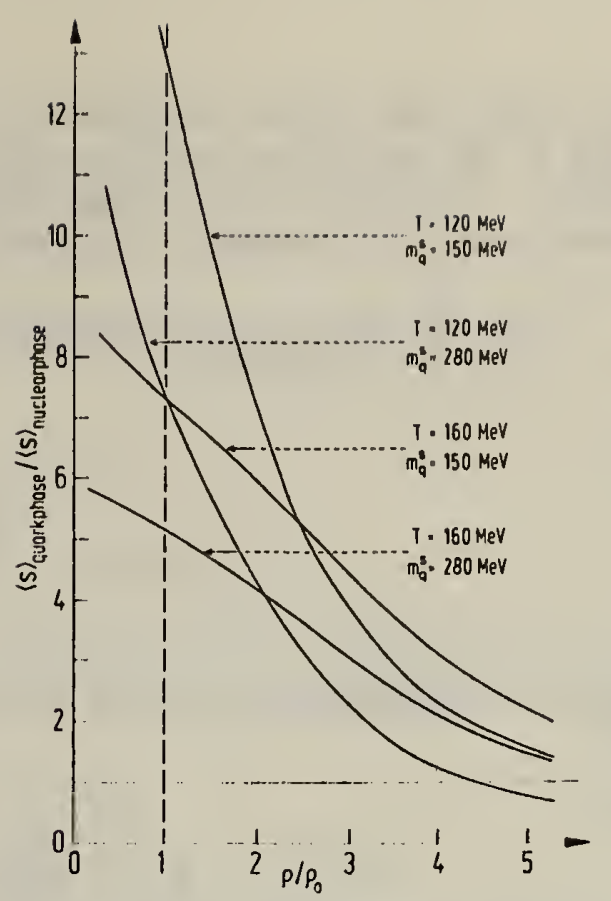

Fig. 7.7 Ratio of strangeness along the transition line between the plasma and the hadronic gas phase as a function of assumed baryon density on the plasma side.

\section{STRANGENESS AS AN EXPERIMENTAL TRIGGER FOR PLASMA DROPLETS}

In the above discussion, the rapid production of strangeness in the plasma phase and its higher statistical abundance as compared with the hadronic gas phase, are the central features of strangeness as a characteristic observable for the quark-gluon plasma. While we have discussed in this work mainly the large plasma domains, we now will turn our attention to "small" plasma droplets which may be either created in collisions of light nuclei [55] or perhaps in antiproton annihilations on nuclear targets [2h], [56]. Before turning to these phenomenological details we first derive another effect that further enhances the role of strangeness as a signature for plasma droplets.

As we have described at length in section 4 , exact conservation of quantum numbers, here of the total strangeness, greatly influences the actual partition function. Rewriting eq (4.61) for strange quarks we have the generating partition function

$$
\begin{aligned}
\ln \tilde{z}_{q_{S}} & =g_{S} V \int \frac{d^{3} p}{(2 \pi)^{3}}\left\{\ln \left(1+\exp \left[-\beta \sqrt{p^{2}+m^{2}}-\frac{\beta \mu}{3}+i \phi\right]\right)\right. \\
& \left.+\ln \left(1+\exp \left[-\beta \sqrt{p^{2}+m^{2}}+\frac{\beta \mu}{3}-i \phi\right]\right)\right\}
\end{aligned}
$$


where the statistical degeneracy of strange quarks is $g_{s}=2_{s} \times 3_{c}=6$. In eq (8.1) we have included the baryochemical potential associated with strange quarks. The angle $\phi$ is associated with the U(1) group and will allow to ensure exact strangeness conservation.

From eq (8.1) we can extract the partition function of given strangeness s by a simple integration

$$
Z_{n_{s}}(T, V, \mu)=\int_{0}^{2 \pi} \frac{d \phi}{(2 \pi)} e^{-i \phi n_{s}} \tilde{z}_{q_{s}}(T, V, \mu ; \phi)
$$

which in the Boltzmann limit can be carried out analytically. We have in this case

$$
\begin{aligned}
\ln \tilde{q}_{q_{S}} & \approx g_{S} V \int \frac{d^{3} p}{(2 \pi)^{3}} \exp \left[-\beta \sqrt{p^{2}+m^{2}}-\frac{\beta \mu}{3}\right] e^{i \phi} \\
& +g_{S} V \int \frac{d^{3} p}{(2 \pi)^{3}} \exp \left[-\beta \sqrt{p^{2}+m^{2}}+\frac{\beta \mu}{3}\right] e^{-i \phi} \\
& =g_{S} \frac{V T^{3}}{2 \pi^{2}} W(m / T)\left[e^{-\beta \mu / 3} e^{i \phi}+e^{\beta \mu / 3} e^{-i \phi}\right]
\end{aligned}
$$

where as usual $W(x)=x^{2} K_{2}(x)$. $I_{k}(z)$

We now recall the generating series of the modified Bessel functions

$$
e^{z(t+1 / t) / 2}=\sum_{k=-\infty}^{+\infty} t^{k} I_{k}(z), \quad t \neq 0,
$$

We introduce the definitions

$$
\begin{aligned}
t & =e^{-\beta \mu / 3+i \phi}, \\
z=2 Z^{(1)} & =2 g_{S} \frac{V T^{3}}{2 \pi^{2}}\left(\frac{m}{T}\right)^{2} K_{2}\left(\frac{m}{T}\right),
\end{aligned}
$$

where $z^{(l)}$ is the one-particle partition function. For the generating partition function in the Boltzman approximation we have hence 


$$
q_{q_{s}}^{s}=\sum_{k=-\infty}^{+\infty} e^{-\beta \mu k / 3} e^{i k \phi} I_{k}\left(2 Z^{(1)}\right)
$$

The integral (8.2) can now be carried out without further delay

$$
z_{n_{s}}^{s}=e^{-\beta \mu n_{s} / 3} I_{n_{s}}\left(2 z^{(1)}\right)
$$

For finite $n_{s}$ the chemical potential regulates the particle abundances in the conventional fashion. For $n_{s}=0$ we have a very interesting special case [57]

$$
Z_{0}(T, V)=I_{0}\left(2 Z^{(1)}(T, V)\right)
$$

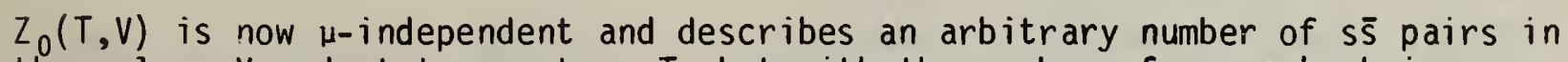
the volume $V$ and at temperature $T$, but with the number of $s$-quarks being exactly equal to that of $s$ quarks. However, having used the Boltzman limit, we have constrained the validity of eq $(8.8)$ to temperatures not much larger than the strange quark mass; only then is the expansion (8.3) valid, i.e., the phase space sufficiently thinly populated allowing the neglect of quantum symmetry effects.

Next we notice that the argument in the $I_{0}$ function is the number of strange and antistrange quarks, computed as if we had neglected the influence of exact strangeness conservation. In the limit that this number is large we can employ the asymptotic expansion

$$
I_{0}(z) \sim \frac{e^{z}}{\sqrt{2 \pi z}}\left(1+\frac{1}{8 z}+\ldots\right)
$$

to recover the usual result

$$
\ln Z_{0}(T, V) \underset{V+\infty}{\rightarrow} 2 Z^{\left({ }^{1}\right)}(T, V)=Z_{S}^{\left({ }^{1}\right)}(T, V)+Z_{\bar{s}}^{\left({ }^{1}\right)}(T, V)
$$

In the above equation we have the volume $V$ as the only quantity that controls the argument of the partition function at fixed temperature.

The actual number of s-pairs present in the plasma is

$$
\left\langle n_{s}\right\rangle=\lambda \frac{\partial}{\partial \lambda} \ln I_{0}\left(\left.\lambda^{\left.1 / 22 Z^{(1)}\right)}\right|_{\lambda=1}=\frac{I_{1}\left(2 Z^{\left({ }^{1}\right)}\right)}{I_{0}\left(2 Z^{(1)}\right)} Z^{\left({ }^{1}\right)}(T, V)\right.
$$


where we note the appearence of a suppression factor

$$
n(T, V)=\frac{I_{1}\left(2 Z^{(1)}\right)}{I_{0}\left(2 Z^{(1)}\right)}
$$

This factor is shown in figure 8.1 for $T=160 \mathrm{MeV}$ and strange quark mass $m_{s}=160 \mathrm{MeV}$ as a function of the volume. The volume is measured in terms of the elementary hadronic volume $V_{h}=\frac{4 \pi}{3}(1 \mathrm{fm})^{3}$. The important aspect of this result is that as the volume goes from $1 / 2 V_{h}$ to $3 V_{h}$, $n$ more than triples. Hence, we expect that in the plasma droplet strangeness would be enhanced by the relaxation of phase space constraints arising for small volumes from the fact that

strangeness is generated in $s \bar{s}$ production which is the physical fact expressed by the quenching factor (8.12).

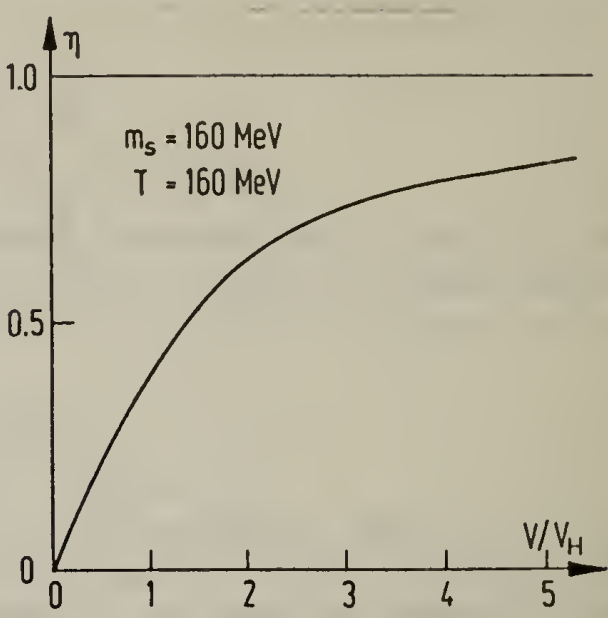

Fig. 8.1 Suppression factor for strangeness production as a function of the reaction volume.

This way we have two effects leading to a significant increase in the strangeness abundance even in small plasma droplets:

(a) The nonlinear volume effect: the abundance of strangeness is not only proportional to $V$ through $z(1)$ eq $(8.10)$, but in addition there is the disappearance of the phase space quenching factor $n$ eq (8.12) for volumes exceeding $V_{H}$. Even for small droplets with $V \approx 2 V_{H}$ this effect leads to an enhancement by a factor $\sim\left(V / V_{h}\right)^{2}=4$.

(b) The word "plasma" implies the equidistribution of the energy into the available degrees of freedom and a lifetime of the droplet of more than $10^{-23} \mathrm{sec}$. As the QCD cross sections indicate (see preceeding sections) this time almost allows reaching the chemical equilibrium state. As discussed in section 7 this means that the strangeness abundance found would be larger by a factor of 2 to 4 than in the equilibrated hadronic gas phase. As the equilibration is also unlikely in the gas phase, we must also expect at least a factor 5 or so more strangeness from the plasma droplet than from the gas phase.

Taken together, both (a) and (b) imply that the strangeness originating from plasma droplets is more than ten times as abundant than that expected from the hadronic gas phase, making the appearance of plasma easily visible as a plasma production threshold is passed in suitable experiments. 
While no systematic experimental information is available as of now, we have found one piece of data which seems to confirm these considerations. Namely, instead of using high-energy nuclear collisions, one can employ antiproton annihilation in nuclei in order to produce a local plasma droplet [56]. We would like to argue that when slow i.e., LEAR antiprotons penetrate into a nucleus the first step in the annihitation process will be the formation of a baryon-number zero fireball, filled with colored gluons and quark-antiquark pairs. As it turns out, this picture allows us to describe satisfactorily the $\pi$-multiplicities in annihilations [58]. In pp reactions such a state would then break up into several mesons, a process that may last sufficiently long to allow the fireball to sometimes collide with one or more of the nearby nucleons in a nucleus. Very likely, this will lead to the absorbtion of some as yet unspecified number of nucleons into the fireball. What do equations of state, cf. section 4, tell us about the physical properties of such a state? We have the energy density of a (relaxed) droplet, eq (4.6):

$$
4 B=E / V=(E / b)(b / V), b=A-1
$$

where $b$ is the baryon number of the droplet, $i . e$. , one less than the total number of nucleons $A$ that have reacted with the incoming antiproton. We also know the total energy $E$ of the fireball,

$$
E=(A+1) m_{N}+E_{k i n}^{\bar{p}}
$$

and hence can solve eq (8.13) for the baryon density $\nu$ of the droplet:

$$
\nu=b / V=4 B \frac{b}{E}=\frac{4 B}{m_{N}} \frac{A-1}{A+1}\left(1+\frac{1}{A+1} E_{k i n}^{\bar{p}} / m_{N}\right)^{-1}
$$

At low energy the last factor containing the kinetic energy of $\bar{p}$ may be dropped. Figure 8.2 shows the "compression" $\nu / \nu_{0}$ as function of $A$, where $\nu_{0}$ as usual is the normal nuclear density in heavy nuclei, $v_{0}=.145 / \mathrm{fm}^{3}$.

We see that the baryon density of the droplet remains similar to that of the normal nuclear matter, i.e., we find no appreciable compression [56b].

Certainly, the quark droplet will disintegrate into A-1 baryons and several mesons. Our understanding of this process is still unsatisfactory but some properties of

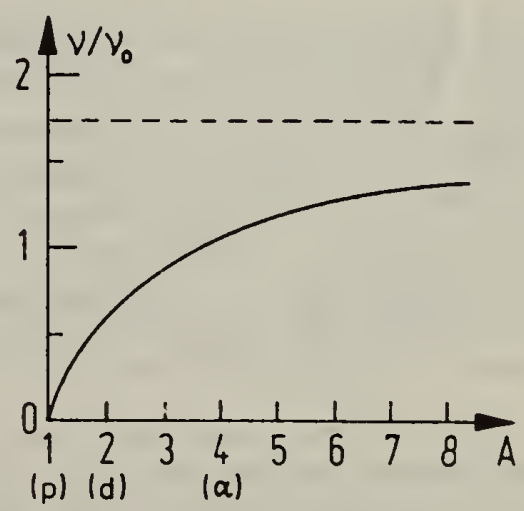

Fig. 8.2 Nuclear matter compression as a function of $A$. 
the emerging particles can nonetheless be estimated. First we observe that inside the low density droplet a temperature of the order of about $160 \mathrm{MeV}$ $(A=1)$ prevails. This value is actually slightly $A$-dependent but will be about $140 \mathrm{MeV}$ even for large $A$, owing to the low baryon density. Particles originating from the disintegration of the droptet will in the event-ensemble show a momentum distribution with characteristic slope ( 1/T). Parallel to this effect we should expect a significant enhancement of the strange particle abundance, as just described.

Apparently, an experiment to observe a plasma droplet would consist of using a strangeness trigger and measuring the momentum distribution of high-momentum so called spectator protons. Such an experiment has actually been carried out for the lightest nuclei. In figure 8.3 we show the results of the $\bar{p}-d$ annihilation taken from reference [59]. Here the event rate as a function of the recoiling proton momentum is shown if the annihilation is accompanied by $K \bar{K}$ production.

Indeed we see a strong enhancement at proton momenta $p>.3 \mathrm{GeV}$ which nicely follows a $T=160 \mathrm{MeV}$ slope. It is very interesting to note that this clear signal seems to disappear in the background when the KK trigger is not used (see fig.? of reference [60]). Another confirmation of this interpretation is obtained by considering the reaction

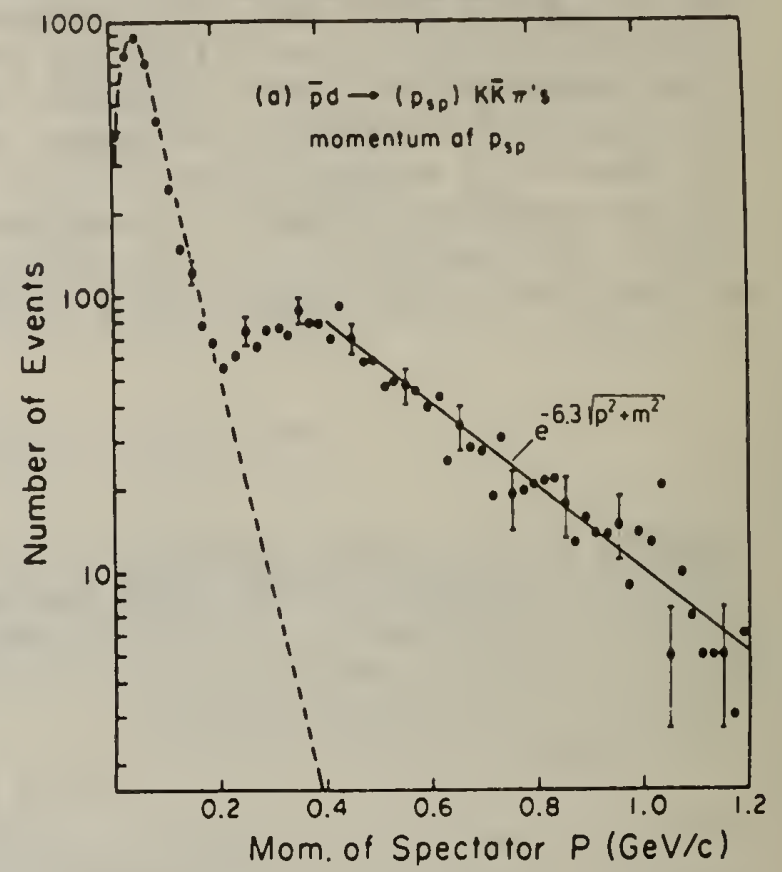

Fig. 8.3 Momentum distribution of spectator protons in coincidence with strangeness production.

$$
\overline{\mathrm{p} d}+\Lambda+X
$$

The strangeness is now attached to the nucleon and the reaction is self analyzing in the sense that the recoiling particle has the trigger quantum number. Indeed, in reference [61] Oh and Smith record that the $\Lambda p_{\perp}$ spectrum is identical to their $p$ spectrum in the bump above $p_{1}>.3 \mathrm{GeV}$. Recent measurements of the reaction (8.16) [62] present also an alternative interprtation in terms of mass shell K-exchange which, however, seems to fall short of the data. Another experimental evidence against the $K$-exchange mechanism is the anomalous enhancement of "K-d reactions" when the spectator momentum exceeds $200 \mathrm{MeV} / \mathrm{c}$ [63].

We can thus conclude that in the $p$ - $d$ reaction a first signal for the annihilation on two nucleons leading to a $b=1$ plasma droplet may have been seen. It would be of great interest to see if a similar signal can be obtained, e.g., in $\bar{p}-\alpha$ annihilations. Here in particular a $4 \pi$ geometry would be of great help in order to select events in which all three remaining nucleons share the annihilation energy. A simultaneous enhancement of the $s-$ yield would give a confirmation of the presented arguments. 
Our aim has been to obtain a description of highly excited hadronic matter. By considering matter in kinetic and chemical equilibrium we have been able to develop a thermodynamic description valid for high temperatures and different chemical compositions. In the present work we have described two physically different domains: first, the hadronic gas phase, in which individual hadrons can exist as separate entities, but are sometimes combined to larger hadronic clusters; and second, the quark-gluon plasma, where individual hadrons dissolve into one large cluster consisting of the hadron constitutents.

In order to obtain a theoretical description of both phases we have used some "common" knowledge and plausible interpretations of the currently available experimental observations. In particular, in the case of the hadronic gas we have completely abandoned a more conventional Lagrangian approach in favor of a semi-phenomenological statistical bootstrap model of hadronic matter that incorporates those properties of hadronic interaction that are, in our opinion, the most important.

In particular, the attractive interactions are included through the rich exponentially growing hadronic mass spectrum $\tau\left(m^{2}, b\right)$ while the introduction of the finite volume of each hadron is responsible for an effective short-range repulsion. We neglect quantum statistics in the hadronic gas phase since a quantitative study has revealed that this is allowed above $T \approx 50 \mathrm{MeV}$. But we allow particle production, which introduces a quantum physical aspect into the otherwise classical theory of Boltzmann particles.

Our considerations lead us to the equations of state of hadronic matter which reflect what we have included in our considerations. It is the quantitative nature of our work that allows a detailed comparison with experiment. It is important to observe that the predicted temperatures and mean transverse momenta of particles agree with the experimental results available at $E_{k}, 1 \mathrm{ab} / A=2 \mathrm{GeV}$ [BEVELAC] and at $100 \mathrm{GeV}$ [ISR] as far as a comparison is pérmitted [4a].

The internal theoretical consistency of our description of the gas phase leads us in a straightforward fashion to the postulate of a first order phase transition to the quark-gluon plasma. This second phase is treated by a quite different method; in addition to the standard Lagrangian quantum field theory of "weakly" interacting particles at finite temperature and density, we also introduce the phenomenological vacuum pressure and energy density $B$. This term is required in a consistent theory of hadronic structure. It turns out that $\mathrm{B}^{1 / 4} \sim 190 \mathrm{MeV}$ is just, to within $20 \%$, the temperature of the plasma phase before condensation into hadrons. This is similar to the maximal hadronic temperature $\mathrm{T}_{0} \cong 160 \mathrm{MeV}$.

Perhaps the most interesting aspect of our work is the realization that the transition to the quark-gluon plasma matter will occur at a very much lower baryon density for highly excited hadronic matter than for matter in the ground state $(T=0)$. Using the currently accepted value for $B$ we find that at $v \sim 2-3 v_{0}, T=150 \mathrm{MeV}$, the plasma phase may indeed already be formed. 
These plasma characteristics were used to evaluate both the radiation cooling of the plasma by the emission of pions, and the conditions under which a plasma seed will grow in a high energy nuclear collision. Using the above value $T=150-160 \mathrm{MeV}$ as the phase transition temperature we find that 20 $50 \mathrm{GeV} /$ nucleon on a fixed target should suffice to lead to a quark-qluon plasma. The frequency of such events is controlled by the probability that a plasma seed of adequate size be formed early in the collision. A plasma seed is needed since the hadron cross sections are too small to lead to energy confinement for the required length of time. The cross sections in a plasma can be much larger since the phase space there is much larger than in the hadronic gas phase owing to the absence of the color constraint; for example, a 3 quark system in the plasma has 27 color states while only the color singlet is permitted in the hadronic phase.

We also discuss in a somewhat speculative manner the phase transition plasma + hadronic gas in order to estimate the parameters $\mu, T$ needed in the study of possible signatures of the plasma in high energy nuclear collisions.

One of the possible signatures is the strangeness production in the plasma. We show that it has a sufficiently large reaction rate for the strangeness abundance in the plasma to reach chemical equilibrium during the lifetime of the plasma. The subsequent depletion of the strangeness during the plasma hadronization as well as its preferred hadronization channels have not yet been studied in detail. However, only if the plasma hadronization is an extremely slow process, lasting on the order of $10^{-22} \mathrm{sec}$, a significant depletion of the high $s$-abundance created at the maximal temperature reached in the collision can be anticipated. As shown in figure 8.3 the invariant rates drop quite rapidly with decreasing temperature, leading to a rapid increase of the equilibrium time constant $\tau$. Hence the strangeness abundance decouples from the equilibrium and remains a witness of the hot collision period. We have further shown that strangness may be a useful trigger on plasma formation.

While we can not yet discuss in detail the abundance of multi-strange antihadrons which are influenced also by the possible strange bound states in the plasma, it is apparent from our results that the measurement of production cross sections of antistrange baryons already could be quite helpful in the observation of the phase transition. The high suppression of these degrees of freedom in the hadronic gas phase is not maintained in the plasma phase where $\bar{s}$ abundance is larger than $\bar{u}, \vec{a}$ abundance. A measurement of the relative $\mathrm{K}^{+} / \mathrm{K}^{-}$ yield, while indicative of the value of the chemical potential in a hot nuclear gas may carry less specific information about the plasma. The $\mathrm{K} / \pi$ ratio may also contain relevant information. However, since the $\pi$ originates from diverse sources its abundance is controlled by the total entropy created in $\mathrm{N}-\mathrm{N}$ collisions. Hence, it will be much more difficult to decipher the message. Perhaps a steep rise of $K / \pi$ ratio at high $p_{\perp}$ could be helpfull.

On the other hand it appears that the abundances of otherwise quite rare strange hadrons will be enhanced, on the one hand by the relative high phase space density of strangeness in the plasma, on the other hand in view of the attractive $S S-Q C D$ interaction in the $\overline{3} c$ and $\bar{s} s$ in $1_{C}$ channels. Hence we should search for the strangeness abundance in the yields of particles like 
$\Xi, \bar{\Xi}, \Omega, \bar{\Omega}, \phi$, rather than in the K-channels. It may be that such experiments would uniquely determine the existence, and eventually the characteristics, of the phase transition to the quark-gluon plasma.

It is important to appreciate that the experiments discussed above would certainly be quite complementary to the measurements utilizing electromagnetically interacting probes, e.g., dileptons, direct photons. Strangeness based measurements have the advantage that they are much more abundant than the electromagnetic particles since they involve the observation of a strongly interacting particle ( $s, \bar{s}$ quark) which happened to be a direct constituent of the hot plasma phase.

\section{ACKNOWLEDGMENTS}

While much of the work is still left to the future, we would like to thank all who have through collaboration or stimulating discussions helped in the development of this research field: H.-Th. Elze, W. Greiner, R. Hagedorn, P. Koch, B. Merl ler, H. Rafelski, and G. Staadt. One of us (J.R.) would like to thank Prof. R. Bock for his hospitality at the GSI laboratory where much of this work has been done. Several research periods at CERN-Theory Division by one of us (J.R.) have contributed essentially to the theoretical developments presented here. 


\section{REFERENCES}

1. a. Workshop on Future Relativistic Heavy Ion Experiments, Proceedings edited by R. Stock and R. Bock, GST 81-6, Orange Report 1981.

b. Workshop on Quark Matter Formation and Heavy Ion Collisions, Proceedings edited by M. Jacob and H. Satz, World Scientific Publ. Co., Singapore 198?.

2. An incomplete list of quark-gluon plasma papers includes:

a. B. A. Freedman and L. D. McLerran, Phys. Rev. D16 (1977) 1169;

b. S. A. Chin, Phys. Lett. 78B (1978) 552;

c. P. D. Morley and M. B. KisTinger, Phys. Rep. 51 (1979) 63;

d. J. I. Kapusta, Nucl. Phys. B148 (1979) 461;

e. O. K. Kalashnikov and V. V. KiTmov, Phys. Lett. $88 B$ (1979) 328;

f. F. V. Shuryak, Phys. Lett. $81 \mathrm{~B}$ (1979) 65; also Phys. Rep. 61 (1980) 71 ;

g. 3. Rafelski and R. Hagedorn "From Hadron Gas to Quark Matter II, " in Thermodynamics of Quarks and hadrons, Ed. H. Satz, North Holland, Amsterdam 1981.

h. J. Rafelski, H.-Th. Elze, and R. Hagedorn, "Hot Hadronic and Quark Matter in p-Annihilation on Nuclei," CERN Preprint TH2912 (1980), in Proceedings of 5 th European Symposium on Nucleon-Antinucleon Interactions, Bressanone 1980, CLEUP, Padua, 1980

3. a. G. Domokos and J. I. Goldman, Phys. Rev. D23 (1981) 203;

b. K. Kajantie and H. I. Mietinnen, Z. Phys. Cg (1981) 341 .

c. K. Kajantie and H. I. Mietinnen, Z. Phys. CI4 (1982) 357.

4. a. J. Rafelski, "Extreme States of Nuclear Matter" in reference [1],

p. 282; also Universitat Frankfurt Preprint UFTP 52/1981;

b. J. Rafelski and B. Maller, Phys. Ref. Lett. 48 (1982) 1066;

c. P. Koch, J. Rafelski, and W. Greiner, Phys. Lett. 123B (1983), 151.

d. J. Rafelski, "Strangeness in Quark-Gluon Plasma," Universitat

Frankfurt preprint UFTP86/198?.

5. 1]. Rafelski, "Hot Hadronic Matter" in New Flavours and Hadron

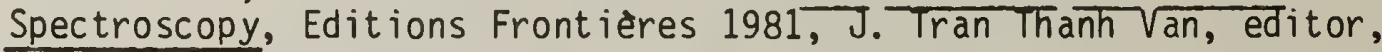
page 619.

6. a. 1]. Kogut, M. Stone, H. Wyld, 1. Shigemitsu, S. Shenker and

D. Sinclair, Phys. Rev. Lett 48 (1982) 114.

b. J. Ellis, "Phenomenology of Unified Gauge Theories" CERN-preprint TH 3174.

c. I. Van Hove, "Very Dense States of Matter in Particle Physics and Early Cosmology," lecture at the Institut-Lorentz, 1981/1982, Leiden, Netherl ands.

7. a. V. A. Rubakov, JETP Lett 33 (1981) 699, and Nucl. Phys. B203 (1982) 311.

b. C. G. Callan, Jr., Nucl. Phys. B212 (1983) 391.

c. C. F. Callan, "Catalysis of Baryon Decay," lecture at the Magnetic Monopoles Conference, Racine, Wisconsin, October 1982. 
8. These ideas originate in Hagedorn's statistical bootstrap theory, see:

R. Hagedorn, Supp 1. Nuovo Cimento 3 (1964) 147; and Nuovo Cimento 6 (1968) 311, also

R. Hagedorn, "How to Deal with Relativistic Heavy Ion Collisions," p. 236 in ref. [1].

9. a. R. Hagedorn and J. Rafelski, Phys. Lett. 97B (1980) 136.

b. The extension of statistical bootstrap to finite baryon number and volume has been introduced in:

R. Hagedorn, I. Montvay, and J. Rafelski, Lecture at Erice Workshop "Hadronic Matter at Extreme Energy Density," edited by N. Cabibbo, Plenum Press, New York (1980) p. 49.

C. R. Hagedorn and J. Rafelski, Manuscript in preparation for Physics Reports.

d. R. Hagedorn, Z. Phys. C17 (1983) 265 (CERN Preprint TH-3392).

10. a. A. Chodos, R. L. Jaffe, K. Johnson, C. B. Thorn, V. F. Weisskopf, Phys. Rev. D9 (1974) 3471.

b. K. Johnson, Acta Phys. Polon. B6 (1975) 865; and

c. T. de Grand, R. L. Jaffe, K. Johnson, and J. Kiskis, Phys. Rev. D12 (1975) 2060.

11. a. R. Anishetty, P. Koehler, and L. McLerran, Phys. Rev. D22 (1980) 2793.

b. J. D. Bjorken, Phys. Rev. D27 (1983) 140.

12. M. Danos and I. Rafelski, "Formation of Quark-Gluon Plasma at Central Rapidity," University of Frankfurt preprint UFTP 94/1982.

1.3. a. S. A. Chin, Phys. Lett 119B (1982) 51.

b. K. Kajantie and L. McLerran, "Energy Densities, Initial Conditions and Hydrodynamic Equations for Ultrarelativistic Nucleus-Nucleus Collisions," University of Helsinki Report HU-TPT-82-30.

14. M. Danos and J. Rafelski, Phys. Rev. D27 (1983) 671.

15. S. Gasiorowicz and J. L. Rosner, "Hadron Spectra and Quarks," Am. J. Phys. 49 (1981) 954.

16. F. S. Abers and B. W. Lee, "Gauge Theories," Phys. Rep. 9C (1973) 1.

17. P. Soding and G. Wolf, "Experimental Evidence on QCD," DESY-Report 81 013; (1981); also, Ann. Rev. Nuc. Part. Sci. (1982).

18. a. P. Langacker and H. Pagels, Phys. Rev D19 (1979) 2070, and references therein.

b. S. Narison, N. Paver, E. de Rafael, and D. Treleani, Nuc?. Phys. B212 (1983) 365.

19. R. D. Viollier and J. Rafelski, "Quarkonium Spectra in the Framework of Quantum Chromodynamics," Helv. Phys. Acta 53 (1980) 352. 
- A. Martin, "Heavy Quark Systems," CERN-TH 3162, appeared in Proceedings of the Int. Conf. on High Energy Physics, Lisbon, July 1981.

21. a. H. D. Politzer, Phys. Rev. Lett. 30 (1973) 1346.

b. D. Gross and F. Wilczek, Phys. Rev. Lett. 30 (1973) 1343.

c. W. Marciano and H. Pagels, "Quantum Chromodynamics," Phys. Rep. 36C (1978) 137.

22. K. Johnson, "A Simple Model of the Ground State of Quantum Chromodynamics," SLAC-Publ 2436 (1979), in AIP Conf. Proc. No. 59, Am. Inst. of Phys., N.Y. 1979.

23. J. Rafelski, "Particle Condensates in Strongly Coupled Quantum Field Theory," UFTP Preprint 67/1981 in Quantum Electrodynamics of Strong Fields, edited by W. Greiner, D. Reidel PubT. Co. (1981).

24. P. Hasenfratz, R. R. Horgan, J. Kuti, and J. M. Richard, Phys. Lett. 95B (1980) 299.

25. See, e.g., M. A. Shifman, Z. Phys. 69 (1981) 347 and references therein.

26. G. Peressutti and B.-S. Skagerstam, "Hydrodynamics and the Bag Model," Phys. Ref D18 (1978) 4304.

27. See, e.g., R. R. Feynman, "Statistical Mechanics," W. A. Benjamin, Inc. 1972.

28. H.-Th. Elze, W. Greiner, and J. Rafelski, "The Relativistic Ideal Fermi Gas Revisited," J. Phys. G6 (1980) L149.

29. a. See, T. Barnes, F. E. Close, and F. deViron, "QQg Hermaphrodite mesons in the MIT bag model," Rutherford preprint RL-82-088 T.311 and references therein.

b. D. Robson, "Toroidal Bags," Z. Physik C3 (1980) 199.

30. a. S. J. Lindenbaum, in Proceedings XVIth Renconte de Moridae - "New Flavours and Hadron Sopectroscopy, " edited by J. Tran Thanh Van, Frontieres (1981) p. 187.

b. See, J. Donoghue, and H. Gomm, Phys. Lett. 112B (1982) 409, and references therein.

c. D. G. Aschman, (Crystal Ball collaboration) "Possible Gluonium States in Radiative $\psi$ Decay," in Proc. of XVIIth Recontre de Moriond, Workshop on New Flavors, Les Arcs, France, 1982.

31. P. Carruthers, "Role of the Phonon in the QCD Plasma," Los Alamos preprint LA-UR-83-130.

32. C.-G. Kallman and C. Montonen, Phys. Lett. 115B (1982) 473.

33. H.-Th. Elze, W. Greiner, and J. Rafelski, Phys. Lett. 124B (1983) 515.

34. a. K. Redlich and L. Thurko, Z. Phys. C5 (1980) 201,

b. L. Thurko, Phys. Lett. 104B (1981) 153. 
35. B. Mưller and J. Rafelski, Phys. Lett 116B (1982) 274.

36. For a discussion of the Haar measure we recommend $G$. Rosen, "Formulations of Classical and Quantum Dynamical Theory, "Academic Press, New York 1969, Appendix C; or J. P. Elliot and P. G. Dawber, "Symmetry in Physics," Vols. 1/2, McMillan, London 1979, Appendix A.4.3.

37. G. B. Yodh, "Review of Recent Developments in Cosmic Ray Experiments at Very High Energies," p. 213-236 in reference [1b] and references therein.

38. M. A. Faessler, Ann. Physics, (N.Y.) 137 (1981) 44.

39. UA1-Collaboration-CERN, G. Arnison, et al., Phys. Lett. 123B (1983) 108.

40. M. Danos and R. K. Smith, unpublished; see also, A. Ausden, J. N. Ginochio, F. H. Harlow, J. R. Nix, M. Danos, E. E. Halbert, and R. K. Smith, Phys. Rev. Lett. 38 (1977) 1055.

41. R. V. Gavai and F. Karsch, "On the Order of the Finite Temperature Deconfinement Phase Transition in $Z(2)$ and $Z(3)$ Lattice Gouge Theories," CERN preprint TH3524.

42. For some time it has been suggested in the early literature that the phase transisiton of hadronic matter, like it is the case in SU(2) lattice simulations would be of 2 nd order. We have always maintained that SU(2) results must be taken with utmost care when generalized to SU(3), not only concerning the order of the transition, but also the value of the critical temperature. The value of the critical temperature is greatly influenced by the number of degrees of freedom, which more than doubles when going from SU(2) to SU(3).

43. A first order phase transition has been conjectured in ref. $[2 \mathrm{~g}, \mathrm{~h}]$ at the time when other schools of thought prevailed. We note here, however, that actually it is possible to avoid a jump in the energy density in such an approach by making quite particular non-general assumptions [9c]. Hence, while in principle the order of the transition remains open, in the semiphenomenological approach taken here one can with some confidence maintain the conjecture of a first order transition.

44. F. Karsch and H. Satz, Phys. Rev. 21D (1980) 1168.

45. R. Hagedorn, "On a Possible Phase Transition Between Hadron Matter and Quark-Gluon Matter" CERN Preprint TH-3207.

46. B. L. Combridge, Nucl. Phys. B151 (1979) 429.

47. G. Saadt, J. Rafelski, and W. Greiner, "High Energy $\gamma^{\prime} s$ from sis Annihilation in Nuclear Collisions," in preparation.

48. M. Jezabeh and J. Rafelski, unpublisher.

49. T. S. Biro, IJ. Zimanyi, Phys, Lett. 113B (1982) 6. 
50. From the study of the process $q \bar{q}+s \bar{s}^{-}$it was initially concluded [49] that strangness would not saturate. The authors of ref. [49] have since corrected their calculations: T. S. Biro, J. Zimanyi, Nucl. Phys. A395 (1983) 525 and agree now in their results with those given here, as originally presented in ref. [4b].

51. P. Koch, Diploma Thesis Frankfurt 1983 (unpublished).

52. A. Z. Mekjian, Nucl. Phys A384 (1982) 492.

53. P. Koch and J. Rafelski, to be published.

54. M. Bourquin et al, Nuc1. Phys B153 (1970) 13.

M. Bourquin et al, Z. Phys. C5 (1980) 275.

55. Oxygen impinging at PS-energies (9-13GeV) on heavy targets is actively considered as a next step in the study of hot nuclear matter. See the GSI proposal reproduced in ref. (1b) page 557.

56. a) J. Rafelski, Phys. Lett. $91 B$ (1980) 281.

b) J. Rafelski, "Quark-Gluon Plasma in p - Annihilation on Nuclei," UFTP preprint 76/1982; in Proceedings of Workshop on Physics at LEAR, Erice, May 1982.

57. J. Rafelski and M. Danos, Phys. Lett 97B (1980) 279.

58. B. Muller and J. Rafelski, Phys. Lett 116B (1982) 274.

59. B. Y. Oh, P. S. Eastman, Z. Ming Ma, D. L. Parker, G. A. Smith and R. J. Sprafka, Nucl. Phys. B51 (1973) 57.

60. P. S. Estman, Z. Ming Ma, B. Y. Oh, D. L. Parker, G. A. Smith nd R. J. Sprafka, Nucl. Phys B51 (1973) 29.

61. B. Y. Oh and G. A. Smith, Nuc 1. Phys. B40 (1972) 151.

62. M. A. Mandelkern, L. R. Price, J. Schulz and D. W. Smith, Phys. Rev. D27 (1983) 19.

63. 0. Braun, V. Hepp, H. Strobbele, and W. Wittek, Nucl. Phys. B160 (1979) 467. 
NBS-114A (REV. 2-80)

U.5. DEPT. OF COMM.

BIBLIOGRAPHIC DATA

SHEET (See Instructlons)

1. PUBLICATION OR REPORT NO.

NBSIR 83-2725

2. Performinz Organ. Report Nod 3. Publlcation Date

June 1983

4. TITLE AND SUBTITLE

\section{PERSPECTIVES IN HIGH ENERGY NUCLEAR COLLISIONS}

\section{AUTHOR(S)}

Johann Rafelski and Michael Danos

6. PERFORMING ORGANIZATION (If joint or other than NBS, see instructions)

7. ContracUGrant No.

NATIONAL BUREAU OF STANDARDS

DEPARTMENT OF COMMERCE

WASHINGTON, D.C. 20234

9. SPONSORING ORGANAZATION NANE AND COMPLETE AODRESS (Street, City, StOTE, ZIP)

10. SUPPLEMENTARY NOTES

[Document describes a computer program; SF-185, FIPS Software Summary, is attached.

11. ABSTRACT (A 200-word or less factual summary of most significant information. If document includes a significant bibliography or literature survey. mention it here)

This report has been prepared as a working document for the conception of a research facility devoted to the study of high energy nuclear collisions. Different aspects of hadronic physics to be studied in nuclear collisions are selected, with emphasis placed on the properties and nature of the quark-gluon plasma, the formation of the plasma state in the central region and its anticipated lifetime, and the observability and strangeness content of this new form of nuclear matter.

12. KEY WORDS (Six to tweive entries; alphabetical order: capitalize only proper names; and separate key words by semicolons)

elementary particles; gluon; hadron; nuclear collisions; nuclear plasma; quark

\section{AVAILABILITY}

E为 Unlimited

$\square$ For Official Distribution. Do Not Release to NTIS

$\square$ Order From Superintendent of Documents, U.S. Government Printing Office, Washington, D.C. 20402.

[X Order From National Technical information Service (NTIS), Springfield, VA, 2216i

14. NO. OF

PRINTED PAGES

15. Price

$\$ 11.25$ 


